\title{
Republic of Mozambique: Third Review Under the Policy Support Instrument-Staff Report; Staff Supplement; and Press Release
}

In the context of the third review under the Policy Support Instrument with the Republic of Mozambique, the following documents have been released and are included in this package:

- $\quad$ The staff report for the Third Review Under the Policy Support Instrument, prepared by a staff team of the IMF, following discussions that ended on November 2, 2008, with the officials of the Republic of Mozambique on economic developments and policies. Based on information available at the time of these discussions, the staff report was completed on December 23, 2008. The views expressed in the staff report are those of the staff team and do not necessarily reflect the views of the Executive Board of the IMF.

- $\quad$ A staff supplement on the joint IMF/World Bank debt sustainability analysis.

- $\quad$ A Press Release summarizing the views of the Executive Board.

The documents listed below have been or will be separately released.

Letter of Intent sent to the IMF by the authorities of the Republic of Mozambique*

Memorandum of Economic and Financial Policies by the authorities of the Republic of Mozambique* Technical Memorandum of Understanding*

Poverty Reduction Strategy Paper

Joint Staff Advisory Note of the Poverty Reduction Strategy Paper

*Also included in Staff Report

The policy of publication of staff reports and other documents allows for the deletion of market-sensitive information.

\author{
Copies of this report are available to the public from \\ International Monetary Fund $\bullet$ Publication Services \\ $70019^{\text {th }}$ Street, N.W. • Washington, D.C. 20431 \\ Telephone: (202) 623-7430 • Telefax: (202) 623-7201 \\ E-mail: publications@imf.org • Internet: http://www.imf.org
}

\section{International Monetary Fund Washington, D.C.}





\title{
INTERNATIONAL MONETARY FUND
}

\section{REPUBLIC OF MOZAMBIQUE}

\section{Staff Report for the Third Review Under the Policy Support Instrument}

\author{
Prepared by the African Department \\ (In consultation with other departments) \\ Approved by Mark Plant and Dominique Desruelle
}

December 23, 2008

- Discussions for the third review of the Policy Support Instrument (PSI) were held in Maputo October 15-30, 2008. The mission team consisted of Mr. Sharer (head), Mr. Staines, Mr. Yang and Ms. Ocampos (all AFR), and Mr. Hallaert (SPR). Mr. Sulemane (OEDAE) participated in the policy discussions. The mission was assisted by Mr. Fischer (resident representative), and Ms. Bosten and Mr. Wane (resident representative office). Mr. Baxter and Mr. Nucifora (World Bank) joined the discussions.

- The mission met with the Prime Minister; the Ministers of Finance, Justice, Energy, Mineral Resources, Industry and Trade, Civil Service, and State Administration; the Governor of the Bank of Mozambique (BM); other senior government officials; and representatives of the private sector, civil society and the donor community.

- The Executive Board approved a three-year arrangement under the PSI on June 18, 2007 and concluded the second review on May 28, 2008. The third review evaluates performance to end-June 2008. All the quantitative and structural assessment criteria (ACs) and benchmarks have been met.

- In the attached Memorandum of Economic and Financial Policies (MEFP), the authorities review program performance in the first half of 2008 and outline their policies for the remainder of 2008 and for 2009 . The authorities request modifications to the AC on reserve money for end-December 2008.

- Since taking office in 2005 , the government has demonstrated a firm commitment to reform. Municipal elections took place in November 2008. Provincial elections were postponed to the time of the next presidential elections in 2009. 


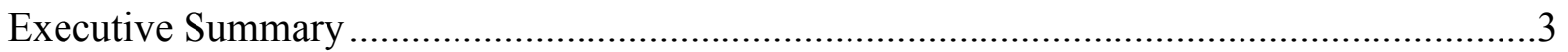

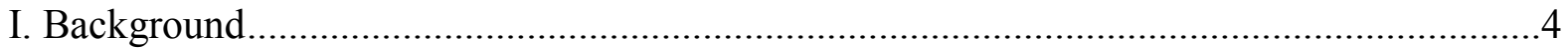

A. Recent Developments ........................................................................... 4

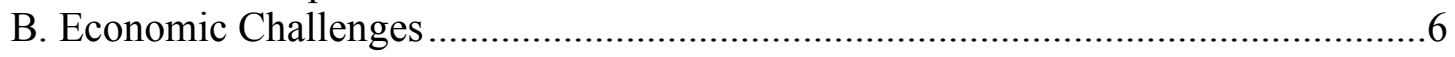

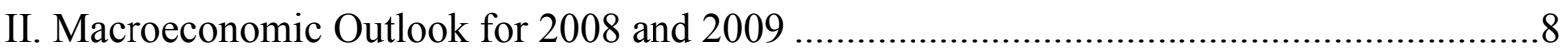

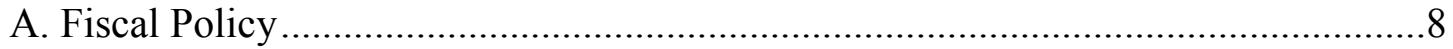

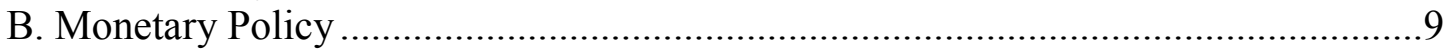

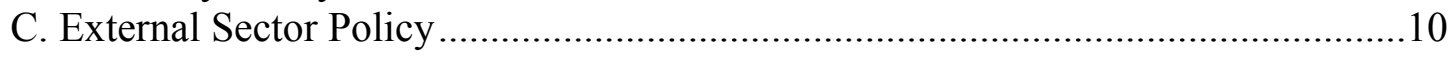

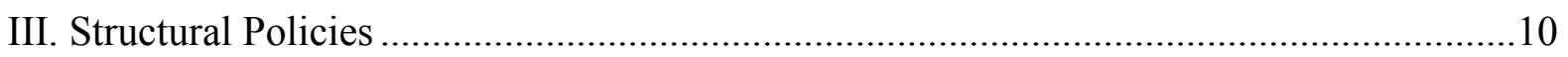

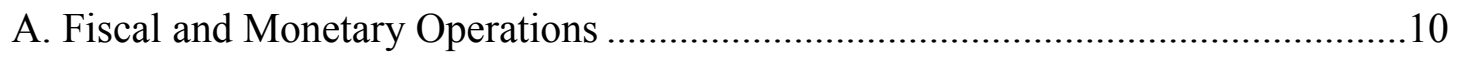

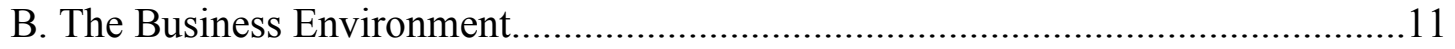

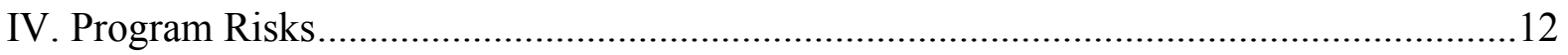

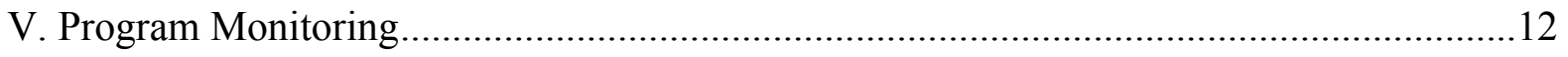

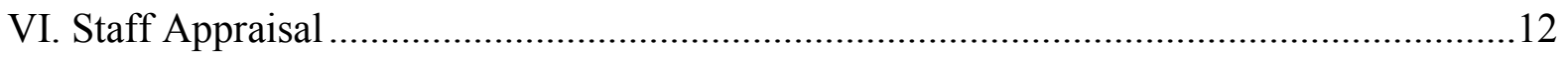

Tables

1. Selected Economic and Financial Indicators, 2007-11 .............................................14

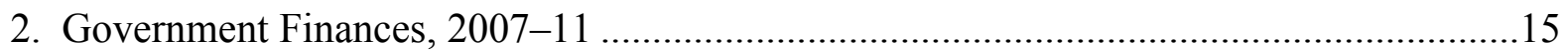

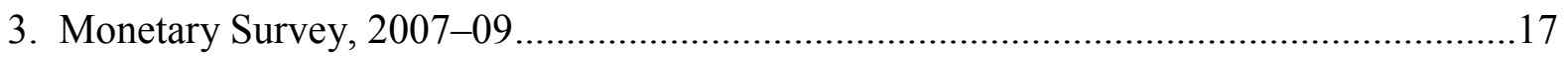

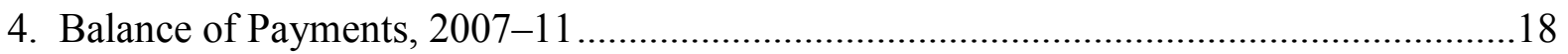

Figure

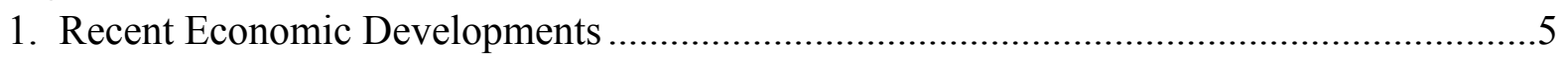

Box

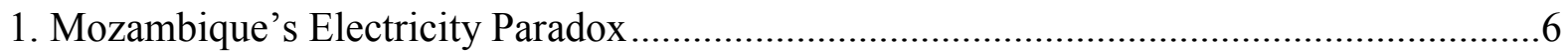

Appendix

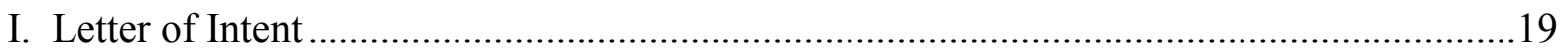

Attachment I. Memorandum of Economic and Financial Policies of the Government of Mozambique for the Third Review of the PSI Supported-Program 21

Attachment II. Technical Memorandum of Understanding on Selected Concept, Definitions, and Data Reporting Under Mozambique's Policy Support Instrument Program 


\section{EXECUTIVE SUMMARY}

Mozambique is meeting the macroeconomic objectives of its medium-term development strategy and program performance to end-June 2008 has continued to be satisfactory. It is also expected to meet the program targets for end-December 2008, except for the one on reserve money, for which the government proposes a modification to accommodate the impact of higher import prices on inflation.

The external environment has presented Mozambique with major challenges. Higher fuel and food import prices in mid-year increased inflation and exposed social vulnerabilities. To mitigate the social impact, the government introduced additional measures in June, which were financed by cuts in non-priority expenditures and additional aid. Output in 2008 has also been adversely affected by disruptions to energy supply, mainly to the aluminum industry, Mozambique's primary exporter.

Mozambique is vulnerable to the global financial crisis and economic slowdown. Lower import prices will help reduce inflation and likely benefit the trade balance. However, lower export volumes and reduced private capital inflows are expected to reduce economic growth. The turbulence could also spread to the financial system despite its limited integration into global financial markets. The government's policy response will need to be carefully tailored to the specific nature of the shock and its impact on domestic demand.

Economic prospects remain strong, but have weakened. Projections for real GDP growth, have been reduced for both 2008 and 2009. However, as international prices decline, inflation should return to single digits in 2009 and beyond.

The government remains committed to prudent fiscal and monetary policies. The fiscal program for 2009 targets a domestic debt repayment of about 0.1 percent of GDP, providing space for a continued significant expansion of private sector credit within an appropriate monetary policy. External reserve import coverage at the end of 2009 will remain comfortable at about 4.5 months of imports.

The government is maintaining the momentum of structural reform. The government is focusing on strengthening policy operations, giving special attention to the tax system and public financial management. The Bank of Mozambique is working to improve policy formulation and implementation and to deepen financial markets. The government is also focusing on reforms to support private sector development, management of the country's natural resources, and public sector governance.

The joint IMF-World Bank debt sustainability analysis indicates that the risk of external debt distress remains low. However, total public debt, including domestic debt, could rapidly become unsustainable if a large primary deficit persists, or if output growth slows significantly and permanently. 


\section{BACKGROUND}

1. Mozambique continues to meet the macroeconomic objectives of its mediumterm development strategy. The economy has encountered major challenges from the external macroeconomic environment, but has shown resilience to external prices shocks and to disruptions to power supplies. However, its performance is vulnerable to the effects of the global financial crisis and economic slowdown.

\section{A. Recent Developments}

\section{Overall macroeconomic performance in the first half of 2008 was satisfactory} and all the program's quantitative and structural ACs and benchmarks to end-July 2008 were met (see Figure 1, MEFP Tables 1 and 2).

\begin{tabular}{lrrrrrrr} 
Mozambique: Selected Economic Indicators, 2006-11 \\
\hline & 2006 & 2007 & $2008 \mathrm{~h} 1$ & 2008 & 2009 & 2010 & 2011 \\
& Act. & Act. & Act. & Proj. & Proj. & Proj. & Proj. \\
\hline Real GDP, percent change & 8.7 & 7.0 & 4.0 & 6.5 & 6.2 & 6.5 & 6.6 \\
CPI, per avg., percent change & 13.2 & 8.2 & 10.4 & 10.4 & 7.1 & 6.1 & 4.7 \\
CPI, eop., percent change & 9.4 & 10.3 & 10.4 & 9.2 & 7.6 & 4.8 & 5.2 \\
REER, percent change & 1.8 & 6.5 & 9.3 & $\ldots$ & $\ldots$ & $\ldots$ & $\ldots$ \\
Current account balance (before & -15.4 & -15.9 & -13.6 & -21.2 & -19.8 & -18.2 & -17.1 \\
Gross external reserves covera! & 4.4 & 5.0 & 4.8 & 4.6 & 4.5 & 4.5 & 4.6 \\
\hline
\end{tabular}

Sources: Mozambican authorities; and IMF staff estimates and projections.

\section{Output in the first half of 2008 remained strong, though somewhat below}

program projections. This was mainly because of lower electricity production and supply, which had adverse implications for manufacturing output and aluminium exports.

International fuel and food prices surged until mid-2008 to levels significantly higher than the program envisaged. As a result, in the 12 months to September, domestic fuel and food prices rose 38 and 16 percent respectively, pushing overall inflation towards 11 percent. However, core inflation (excluding energy and food-related prices) has fallen below 4 percent. Higher fuel prices had limited impact on output and the impact on the trade balance in the first half of 2008 was smaller than expected because import volumes were lower. ${ }^{1}$

4. Higher domestic fuel and food prices have exposed social vulnerabilities, as large domestic fuel price increases in February 2008 triggered riots. To mitigate the social impact, the government introduced an urban transportation subsidy and suspended further fuel price increases until June. In June, as import price increased again, the government reduced fuelrelated taxes to limit pressures on domestic prices and also launched the Food Production Action Plan (FPAP) to boost domestic food output.

\footnotetext{
${ }^{1}$ International aluminium prices also increased in 2008.
} 
Figure 1. Mozambique: Recent Economic Developments

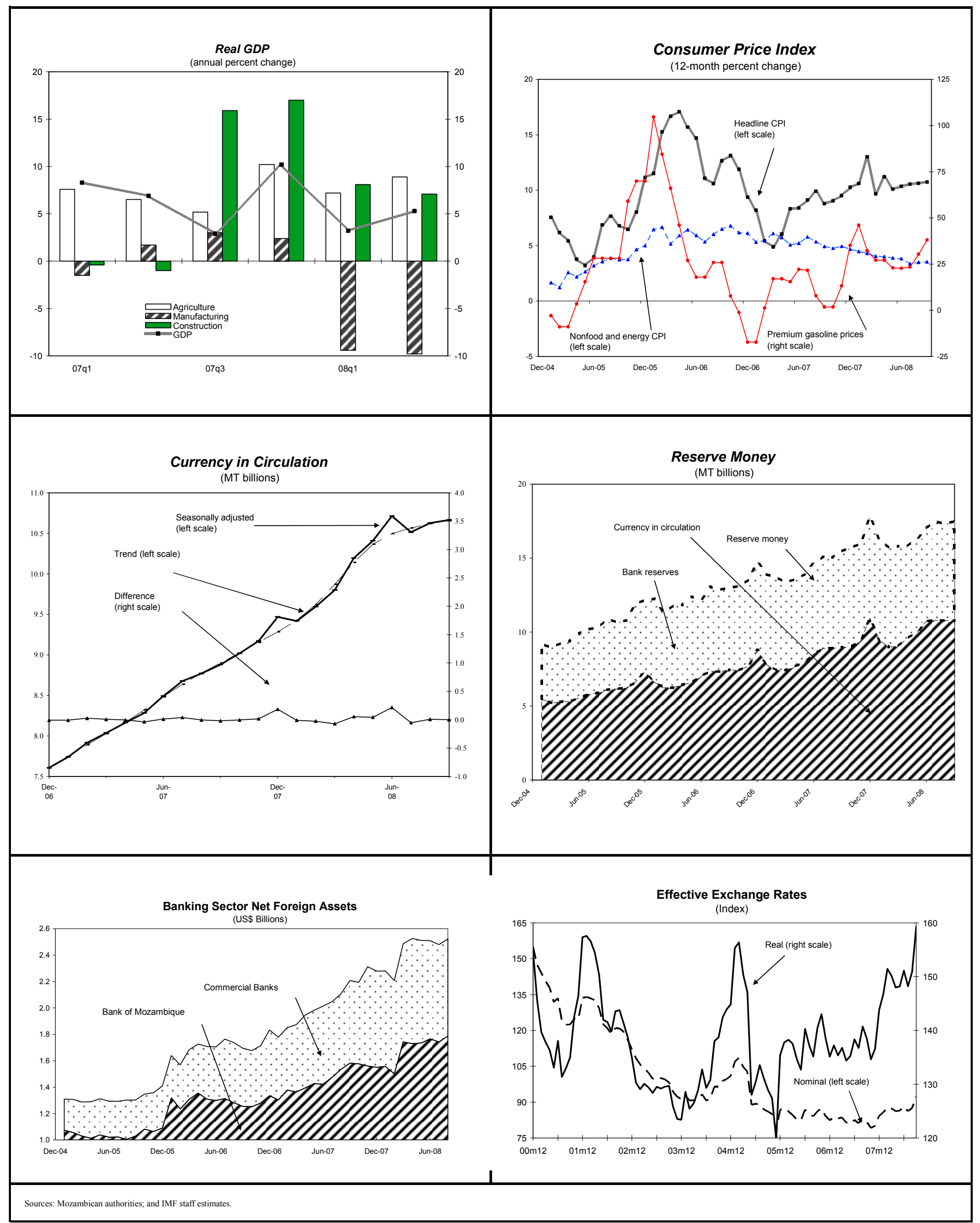


5. Despite these external shocks, macroeconomic policy was generally implemented as envisaged. The government met its end-June target on net domestic financing by a comfortable margin. Despite delays in budget support, revenues were higher than expected because of continued improvements in tax administration, and capital expenditures were lower than budgeted. Reserve money expanded as programmed in the first half of 2008, despite the reduction of the liquidity reserve requirement in April and an unusually large seasonal surge in currency demand. ${ }^{2}$ Private sector credit expanded 28 percent in the 12-months to June, far above expectations. Strong private capital inflows offset pressures on the trade balance and delays in foreign aid, so that external reserve accumulation to June comfortably exceeded program expectations. By the end of August, total banking sector net foreign assets had topped US\$2.5 billion. The exchange rate against the U.S. dollar has remained broadly stable. However, in the context of an appreciating U.S. dollar, Mozambique's real effective exchange rate rose 15 percent over the year to September. ${ }^{3}$

\section{B. Economic Challenges}

6. The core policy objective for Mozambique is to maintain high and sustainable growth over the medium term, so as to reduce poverty and improve the lives of all Mozambicans. This will require continued commitment to prudent macroeconomic policies and to structural reform to make the public sector more effective and to bolster the business environment to attract foreign and domestic investment. However, as it pursues these objectives, Mozambique now faces severe external challenges.

7. Electricity supply constraints are slowing output growth. Although domestic production substantially exceeds consumption, historical patterns of investment in production and transmission, stemming from the days of apartheid and colonization, mean that Mozambique must import power from South Africa. Power shortages in South Africa and inadequate investment in transmission are now constraining supplies to Mozambique (Box 1).

\begin{tabular}{|c|c|c|}
\hline Box 1. Mozambique's El & ctricity Paradox & \\
\hline $\begin{array}{l}\text { Mozambique has } 2,400 \text { megawatts of installed capacity. } \\
\text { This exceeds domestic consumption of about } 1,350\end{array}$ & Mozambique: Energy Highlights & \\
\hline megawatts, of which about 950 megawatts is consumed & Total Installed Capacity (in MW) & 2,400 \\
\hline by the aluminium smelter, Mozal. The remainder is & Of which: Cahora Bassa & 2,000 \\
\hline exported. The output of the Cahora Bassa hydroelectric & Total consumption (in MW) & 1,350 \\
\hline plant, Mozambique main producer of electricity, is & Of which: Mozal & 950 \\
\hline transmitted directly to South Africa. Mozambique then & Energy Trade Balance (in USD million) - 2007 & 121 \\
\hline re-imports energy back from South Africa for its own & Exports & 240 \\
\hline domestic use. As part of South Africa's strategy to cope & Imports & 119 \\
\hline $\begin{array}{l}\text { with its own shortage of power-generation capacity, the } \\
\text { supply of electricity from ESCOM (the South African uti } \\
\text { restricted. Both ESCOM and EDM in Mozambique have } \\
\text { this issue may take several years. }\end{array}$ & $\begin{array}{l}\text { y) to its users including Mozal, has be } \\
\text { gnificant investment programs, but re }\end{array}$ & en \\
\hline
\end{tabular}

\footnotetext{
${ }^{2}$ This was partly because payments to tobacco growers were paid in meticais rather than in Malawian kwacha as was previously the case.

${ }^{3}$ Based on estimates using standard IMF methodology.
} 


\section{The major external risk for Mozambique is its vulnerability to the current global} financial crisis and economic slowdown. The government's policy response will need to be carefully tailored to the specific nature of the shock and its impact on domestic demand.

\section{The major risks are related to how the crisis will affect the terms of trade, export} volumes, capital inflows from donors and the private sector (particularly FDI), and the domestic financial system. Adverse developments in these areas could lead to instability and reduce economic growth. The recent commodity price declines will help reduce inflation in 2009 and will benefit the external current account balance, because merchandise imports exceed exports and because lower aluminium export receipts are likely to be accompanied by lower income outflows. However, the decline in global growth could reduce export volumes and also aid and private capital inflows by more than the modest reduction assumed in the program projections. Lower capital inflows would have a limited impact on the overall balance because of the high import content of the investments financed, but could significantly affect longer-term growth.

\begin{tabular}{|c|c|c|c|c|c|c|}
\hline & 2006 & 2007 & 2008 & 2009 & 2010 & 2011 \\
\hline & Act. & Act. & Proj. & Proj. & Proj. & Proj. \\
\hline Private Capital & 2.7 & 5.7 & 4.5 & 3.6 & 3.8 & 3.6 \\
\hline FDI & 2.1 & 5.3 & 4.7 & 4.4 & 4.4 & 4.3 \\
\hline Net borrowing & 0.6 & 0.4 & -0.2 & -0.8 & -0.6 & -0.7 \\
\hline Borrowing & 3.2 & 14.1 & 3.8 & 3.2 & 3.5 & 3.4 \\
\hline Amortization & -2.7 & -13.7 & -4.0 & -4.0 & -4.1 & -4.0 \\
\hline Aid & 12.8 & 13.1 & 17.6 & 16.2 & 14.5 & 14.5 \\
\hline On-Budget & 12.3 & 12.7 & 17.0 & 16.2 & 14.5 & 14.5 \\
\hline Program, net ${ }^{1}$ & 4.8 & 5.4 & 6.6 & 5.0 & 5.0 & 4.9 \\
\hline Project & 7.5 & 7.3 & 10.5 & 11.2 & 9.5 & 9.6 \\
\hline Off-Budget & 0.5 & 0.4 & 0.6 & 0.0 & 0.0 & 0.0 \\
\hline
\end{tabular}

10. The turbulence could also spread to the domestic financial system, despite its limited integration into global financial markets, because the sector is predominantly foreign owned and also relies heavily on foreign currency denominated deposits and credit. Commercial banks' foreign currency deposits exceed their net foreign assets, but are far less than total net foreign assets, including those of the central bank. The BM will nevertheless need to maintain close supervision of the banking sector.

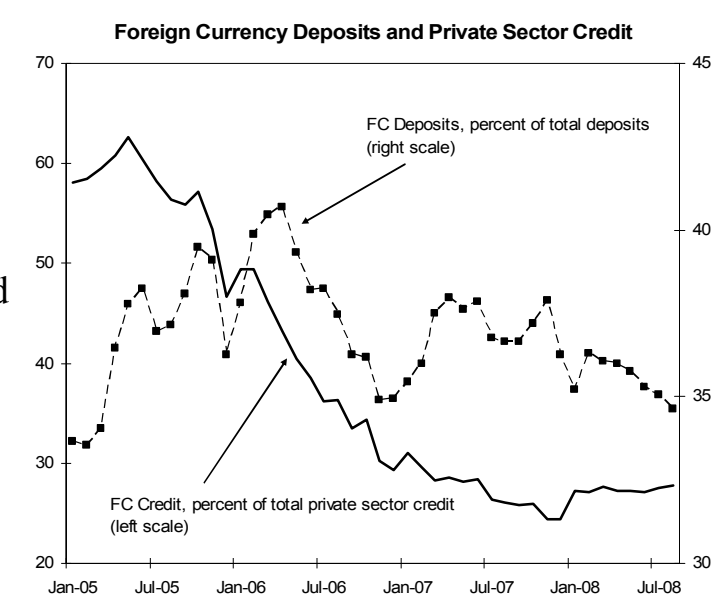




\section{MACROECONOMIC OUTLOOK FOR 2008 AND 2009}

\section{Mozambique's macroeconomic prospects remain solid, though weaker than} previously projected (see Table 1). Reflecting the external challenges, the outlook for real GDP growth has been lowered from 7 to 6.5 percent in 2008 and from 7 to 6.2 percent in 2009. Although robust, this growth is below the average of about 7.6 percent for the previous 5 years. The mid-2008 surge in international fuel and food prices will keep overall inflation at end-2008 above 10 percent, but recent international price declines will help bring it back towards 7 percent in 2009.

\section{A. Fiscal Policy}

12. The government is committed to its medium-term fiscal strategy of making room for private sector credit by avoiding recourse to domestic debt (see MEFP $\{3-5$ and Table 2). Budget implementation for 2008 is on track. The measures introduced in June 2008 to mitigate the social impact of higher import prices cost some 1.2 percent of GDP, but this is expected to be more than offset by cuts in nonpriority spending and additional budget support from the World Bank. Domestic revenue collection is expected to be slightly lower than programmed because of the fuel tax reductions introduced to mitigate the social impact of the fuel price increases. ${ }^{4}$ With somewhat lower than expected 2008 budget support, domestic debt repayment for the whole of 2008 is expected to be modestly lower than programmed. ${ }^{5}$ However, a large advance disbursement of World Bank 2009 budget support will allow the end-December target for net credit to government to be met comfortably.

\section{The government's fiscal program for 2009, as reflected in the budget presented} to Parliament in September, is in line with its medium-term strategy (see MEFP q2426). ${ }^{6}$ With the advance World Bank disbursement, domestic debt repayment in 2009 is expected to be about 0.1 percent of GDP. To meet this target, the government is committed to increasing its domestic revenue by another half percentage points of GDP. Together with the reversal of the June 2008 tax reductions, domestic revenues are thus expected to increase to 16.9 percent of GDP. Measures to mitigate the impact of higher food prices under the FPAP will continue ( 0.5 percent of GDP), and spending in priority areas will be maintained above 65 percent of total spending. Capital expenditures, mostly financed by donors, are expected to rise to 16.3 percent of GDP. The program accommodates an increase in the wage bill to 8.4 percent of GDP to meet the cost of additional education and health hiring and of

\footnotetext{
${ }^{4}$ Revenues in 2008 are expected to be 0.9 percent of GDP lower than programmed. This results from an upward revision of nominal GDP as well as the fuel tax reductions introduced in June.

${ }^{5}$ Disbursement of the World Bank's budget support for 2009 (US\$80 million or about 0.8 percent of GDP) was advanced from 2009 to December 2008 for administrative reasons.

${ }^{6}$ The authorities and Fund staff discussed the macroeconomic framework and the budget via videoconferences in July and August 2008.
} 
wage increases under the new pay policy. The program also incorporates the partial repayment of VAT refund arrears. The urban transportation subsidy has been removed.

\section{B. Monetary Policy}

\section{The BM is committed to a prudent monetary policy geared towards price} stability with reserve money as the intermediate target and in the context of a flexible exchange rate (see MEFP $\uparrow 11-12, \uparrow 32$ and Table 3 ). The BM has revised its money targets to reflect the impact on inflation of fluctuations in international fuel and food prices. In line with Fund staff advice, the BM has expanded monetary growth in the second half of 2008 to accommodate the pass-through of higher import prices to domestic prices. As a result, reserve money is now projected to rise 10 percent by year-end, prompting a revision to the end-December target. ${ }^{7}$ Core inflation is already below the BM's 5 percent medium-term target for overall inflation, and the large recent declines in international fuel and food prices are expected to be passed through to domestic prices. However, in order to avert the second round effects on inflation of the higher import prices during 2008, the BM will constrain reserve money growth to 11 percent in 2009, aiming to reduce overall inflation to about 7 percent by end-2009.

\section{The reserve money expansion will support} continued financial deepening, measured by the share of broad money in GDP, and will allow private sector credit in 2009 to expand about 25 percent. Consistent with Fund staff advice, the BM has continued to lean towards sales of external reserves rather than T-bills, to absorb liquidity so as to avoid crowding out private sector credit. However, Fund staff cautioned that the BM needs to be careful not to further weaken Mozambique's external competitiveness.

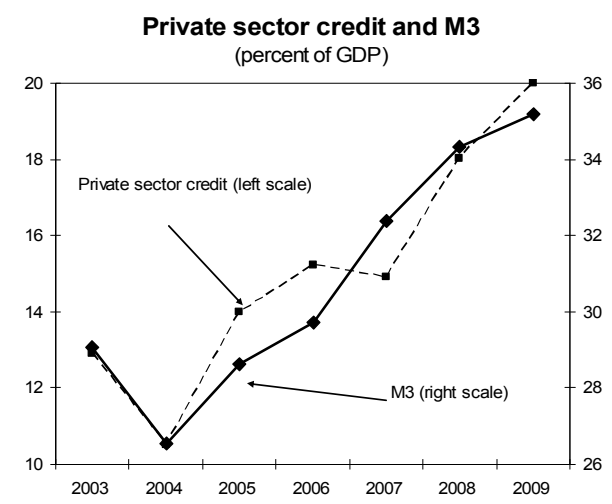

\begin{tabular}{|c|c|c|c|c|c|c|c|c|c|c|}
\hline & \multicolumn{2}{|c|}{2006} & \multicolumn{4}{|c|}{2007} & \multicolumn{4}{|c|}{2008} \\
\hline & Q3 & Q4 & Q1 & Q2 & Q3 & Q4 & Q1 & Q2 & Q3 & $\mathrm{Q}^{*}$ \\
\hline Total sterilization, (MT billions) & 2.2 & 2.8 & 5.3 & 2.4 & 3.7 & 3.8 & 6.2 & 3.7 & 4.9 & 6.6 \\
\hline Net foreign exchange sales & 2.5 & 3.0 & 3.1 & 2.2 & 2.3 & 2.7 & 4.6 & 2.3 & 3.3 & 5.7 \\
\hline Net T-bill issuance & -0.3 & -0.1 & 2.2 & 0.1 & 1.4 & 1.0 & 1.6 & 1.3 & 1.6 & 0.9 \\
\hline Total sterilization, (percent of total) & 100 & 100 & 100 & 100 & 100 & 100 & 100 & 100 & 100 & 100 \\
\hline Net foreign exchange sales & 115 & 105 & 59 & 94 & 62 & 73 & 75 & 64 & 68 & 86 \\
\hline Net T-bill issuance & -15 & -5 & 41 & 6 & 38 & 27 & 25 & 36 & 32 & 14 \\
\hline
\end{tabular}

Sources: Mozambican authorities; and IMF staff estimates. ${ }^{*}$ Projected.

\footnotetext{
${ }^{7}$ Programmed reserve money growth in 2008 took into account the overshooting of reserve money at end-2007 and the reduction in the liquidity reserve requirement in April 2008. However, the increase of currency in circulation during the second quarter was larger than expected. Preliminary data indicate that, though the BM mopped up part of the excess liquidity, reserve money at end-September was higher than programmed.
} 


\section{External Sector Policy}

16. The overall balance of payments in 2008 is expected to be somewhat better than previously envisaged (Table 4) as stronger capital inflows have offset a deterioration in the current account. Private capital inflows were substantially higher than envisaged and the advance World Bank disbursement has mostly offset a sizeable shortfall in project loans. Without the advance disbursement, the overall balance would be US $\$ 40$ million below expectations. The current account has worsened relative to program projections because of a deteriorated trade balance and larger income outflows. Higher commodity prices during the year have boosted import expenditures, especially on fuel, by more than export receipts. Traditional export receipts increased noticeably, but the positive impact on aluminium export receipts has been more than offset by lower export volumes because of disruptions in the supply of electricity.

\section{In 2009, the overall balance of payments is expected to show a small deficit.} Based on October WEO projections at the time of the mission, the current account before grants is expected to improve by 1.4 percentage points of GDP. However, this improvement would be more than offset by private capital and aid inflows, that are cautiously projected to be weaker than previously projected — partly because of the advanced World Bank disbursement. These projections are subject to substantial uncertainties related to international prices for fuel, food and aluminium, the impact of the increased volatility in exchange rate of major currencies, and the impact of the deteriorating international economic environment on private capital inflows and foreign aid. ${ }^{8}$

18. Mozambique's risk of external debt distress remains low. A joint Bank/Fund debt sustainability analysis (see Annex) indicates that the country's external debt will remain well below the indicative thresholds both under the baseline and under various stress tests. However, total public debt, including domestic debt, could rapidly become unsustainable if a large primary deficit persists, or if output growth slows significantly and permanently.

\section{Structural Policies}

\section{A. Fiscal and Monetary Operations (MEFP $q 6-15$ and $q 27-36)$}

19. The government has continued to bolster its domestic revenue effort with a focus on improving tax compliance, making revenue collection more efficient, and expanding the tax base. The revenue authority is moving to integrate revenue collection into the information management system, e-Sistafe (end-September 2009 structural benchmark). It will also allow

\footnotetext{
${ }^{8}$ International commodity prices, especially for fuel, have declined significantly since these projections were made in October. As noted above ( $(9)$, a further general decline in commodity prices will benefit Mozambique's external overall balance in the short term. However, the longer-term impact on growth would likely be negative because of adverse impact on investment and export volumes.
} 
for revenue payments through banks, simplify the collection of income taxes, and strengthen the audit of mega-projects.

20. Firming up public financial management is a priority. Recent reports on the fiscal observance of standards and codes (ROSC) and on public expenditure and financial accountability (PEFA) found significant improvements since previous assessments. The government is preparing a vision paper and an action plan for 2009-11 (end-March 2009 structural benchmark). It is expanding the e-Sistafe system to cover salary payments to all ministries by mid-2009. To strengthen project management, the government will encourage donors to transfer project aid through a multi-currency treasury account starting in early 2009.

21. The government plans to reform the civil service salary and pension policy and the social security system with fiscal sustainability in mind. In September, it approved the medium-term civil service pay policy, to be phased in through 2009, and will prepare a cost estimate by end-August 2009 (structural benchmark). The National Social Security Institute (INSS) has significant unfunded liabilities and the government has drafted a report, which will serve as a basis for making it financially sustainable.

22. The BM has embarked on a medium-term program to adapt monetary policy to better reflect the determinants of price stability, including agreement on a core inflation indicator (end-December 2009 structural benchmark). It is therefore reinforcing its monitoring, analysis and forecasting of macroeconomic and monetary indicators. It is also strengthening liquidity management, by introducing and expanding the use of repos.

23. To buttress banking supervision, the BM will adopt a risk-based framework by end-2009. It will also implement further measures to buttress its internal audit.

\section{B. The Business Environment}

24. With the help of development partners, the government is implementing reforms to support private sector development, natural resource management, and public sector governance (MEFP $₫ 16-21$ and $₫ \mid 37-42$ ).

25. It is stepping up efforts to reduce the cost of doing business, by simplifying taxes for small taxpayers, business licenses, and business inspections (end-March 2009 structural benchmark). By the end of 2009, it also intends to introduce simplified insolvency procedures and a one-stop window to facilitate trade.

\section{The government is pressing ahead to improve management of Mozambique's}

natural resources and is building its capacity to monitor and assess sectoral developments. In close coordination with the private sector and civil society, it will formally initiate the process for adhering to the EITI by end-June 2009 (structural benchmark); measures will then be required over three years for validation. 
27. Initiatives are underway to improve public sector governance. The preparatory study for a public enterprise law has been delayed (end-December 2008 structural benchmark); the government will now submit a draft law to the Council of Ministers by endSeptember 2009 (structural benchmark). In a related area, by the end of 2009, the government will prepare a public-private partnership law designed primarily to manage fiscal and quasi-fiscal risks. It is also working on a government decentralization strategy that will propose a clear legal, regulatory, and institutional framework for raising revenues, assigning spending responsibilities and delineating the functions of sub-national administrative units.

\section{Program Risks}

28. The global financial crisis and economic slowdown present considerable risks for Mozambique's macroeconomic prospects. The government will need to closely monitor developments and be prepared to take actions if needed. In the fiscal area, during an election year, it must resist unrealistic expenditure pressures and distractions from structural reform. The civil service salary and pension reforms and the unfunded liabilities of the INSS pose a risk for fiscal sustainability.

\section{Program Monitoring}

29. The revised program modifies the assessment criterion AC for reserve money for end-December 2008 as noted above (\$14). ACs for end-June and indicative targets for end-December 2009 are proposed and the Technical Memorandum of Understanding has been updated. ${ }^{9}$

\section{StafF ApPraisal}

30. Mozambique's overall macroeconomic performance has been satisfactory. Real GDP growth has been resilient to challenges from the external environment and core inflation has continued to decline. Mozambique has met all the quantitative and structural ACs and benchmarks to end-July 2008. It is also expected to meet the existing program quantitative ACs at end-December 2008 - except for the one on reserve money, given higher inflation arising from higher import prices; staff support the authorities' proposed modification of this AC.

31. The government has demonstrated its ability to mitigate the impact of internal and external shocks with a rapid and flexible policy responses. The government reacted well to the climate shocks of recent years, and in 2008 again responded well to higher fuel and food import prices with a good mix of fiscal and monetary responses.

\footnotetext{
${ }^{9}$ The aid adjuster for net credit to government and net international reserves has been increased from US\$50 million to US\$100 million because of increased uncertainties surrounding the US dollar value of donor disbursements denominated in other currencies.
} 
32. Macroeconomic prospects remain strong, but have been clouded by uncertainties related to the impact of the global financial crisis and economic slowdown. The authorities will need to monitor international and domestic developments closely and respond decisively if necessary. Their readiness to consult with Fund staff on possible measures is welcome.

33. The authorities are committed to the medium-term fiscal strategy of making room for private sector credit by avoiding recourse to domestic financing. The authorities will, however, need to step up their efforts to better manage capital expenditures. It is also vital that the authorities ensure that the civil service wage and pension reforms do not compromise fiscal sustainability. They also need to move quickly to restore the financial sustainability of the INSS.

34. The continued commitment of the authorities to prudent monetary policies with a flexible exchange rate is commendable. The BM's commitment to bringing inflation back to single digits in 2009 is particularly welcome.

35. The authorities have continued to make noticeable progress in strengthening implementation of fiscal and monetary policy. Staff encourage their efforts to mobilize more domestic revenue and improve public financial management. The steps being taken to strengthen monetary policy formulation and implementation are also welcome. In the current global financial crisis, heightened banking supervision is particularly important.

36. The authorities are making real progress on structural reforms to support private sector development, better manage Mozambique's natural resources, and improve public sector governance. Their intention to declare its adherence to the EITI is encouraging, but it will be important to accelerate progress in this critical area.

37. Based on performance to date and Mozambique's continued commitment to program implementation, staff recommend the completion of the third review of the PSI-supported program. 
Table 1. Mozambique: Selected Economic and Financial Indicators, 2007-11

\begin{tabular}{|c|c|c|c|c|c|c|c|}
\hline & \multirow{2}{*}{$\begin{array}{l}2007 \\
\text { Act. }\end{array}$} & \multicolumn{2}{|l|}{2008} & \multicolumn{2}{|l|}{2009} & \multirow{2}{*}{$\begin{array}{l}2010 \\
\text { Proj. }\end{array}$} & \multirow{2}{*}{$\begin{array}{l}2011 \\
\text { Proj. }\end{array}$} \\
\hline & & $\begin{array}{c}\text { Country Report } \\
08 / 220\end{array}$ & Proj. & $\begin{array}{c}\text { Country Report } \\
08 / 220\end{array}$ & Proj. & & \\
\hline & \multicolumn{7}{|c|}{ (Annual percentage change, unless otherwise indicated) } \\
\hline \multicolumn{8}{|l|}{ National income and prices } \\
\hline Nominal GDP (billions of MT) & 207.1 & 226.8 & 234.9 & 255.3 & 266.5 & 303.1 \# & 339.3 \\
\hline Nominal GDP growth & 14.9 & 12.6 & 13.4 & 12.6 & 13.4 & 13.7 & 11.9 \\
\hline Real GDP growth & 7.0 & 7.0 & 6.5 & 7.0 & 6.2 & 6.5 & 6.6 \\
\hline GDP per capita (U.S. dollars) & 397 & 453 & 469 & 474 & 502 & 531 & 568 \\
\hline Consumer price index (annual average) & 8.2 & 7.3 & 10.4 & 5.5 & 7.1 & 6.1 & 4.7 \\
\hline Consumer price index (end of period) & 10.3 & 6.0 & 9.2 & 5.2 & 7.6 & 4.8 & 5.2 \\
\hline \multicolumn{8}{|l|}{ External sector } \\
\hline Merchandise exports & 1.3 & 3.1 & 4.1 & 1.1 & -12.8 & 28.9 & 1.4 \\
\hline Merchandise exports, excluding megaprojects & -16.3 & 4.8 & 11.1 & 8.1 & 6.2 & 7.6 & 7.2 \\
\hline Merchandise imports & 6.1 & 14.5 & 17.5 & 3.5 & 1.1 & 2.9 & 2.4 \\
\hline Merchandise imports, excluding megaprojects & 8.6 & 18.4 & 20.5 & 1.9 & 1.6 & 0.8 & 2.3 \\
\hline Terms of trade & 4.2 & -8.4 & -14.5 & -7.0 & -4.4 & -0.7 & -4.4 \\
\hline Nominal effective exchange rate (end of period) ${ }^{1}$ & 2.2 & $\ldots$ & $\ldots$ & $\ldots$ & $\ldots$ & $\ldots$ & $\ldots$ \\
\hline Real effective exchange rate (end of period) ${ }^{1}$ & 6.5 & $\ldots$ & $\ldots$ & $\ldots$ & $\ldots$ & $\ldots$ & $\ldots$ \\
\hline
\end{tabular}

Money and credit ${ }^{4}$

Net foreign assets

Net domestic assets

Of which: net credit to the government ${ }^{3}$ credit to the economy

Broad money-M3 (12-month percent change)

M2 (12-month percent change)

Velocity (GDP/M2)

Interest rate for 90-day treasury bills (percent; end of period)

(Annual changes in percent of beginning-of-period stock of money-M3, unless otherwise indicated)

Investment and saving

Gross domestic investment

Government

Other sectors

Gross domestic savings (excluding grants)

Government

Other sectors

Current account, before grants

$\begin{array}{rrr}11.8 & 14.8 & 11.6 \\ 13.4 & 2.2 & 8.6 \\ 3.0 & -3.1 & -3.5 \\ 6.5 & 12.1 & 17.1 \\ 25.2 & 17.0 & 20.2 \\ 23.0 & 15.8 & 22.1 \\ 4.8 & 4.6 & 4.5 \\ 14.8 & \ldots & \end{array}$

$\begin{array}{rrr}11.6 & 3.5 & 7.6 \\ 8.6 & 11.0 & 8.5 \\ -3.5 & -0.3 & 2.3 \\ 17.1 & 13.0 & 13.5 \\ 20.2 & 14.5 & 16.1 \\ 22.1 & 14.5 & 15.8 \\ 4.5 & 4.5 & 4.4 \\ \ldots & \ldots & \ldots\end{array}$

(Percent of GDP)

Government budget

Total revenue

Total expenditure and net lending

Overall balance, before grants

Total grants

Overall balance, after grants

Domestic primary balance

External financing (incl. debt relief) ${ }^{3}$

Net domestic financing ${ }^{5}$

$\begin{array}{rrrrrrr}18.0 & 23.9 & 21.9 & 23.7 & 23.7 & 22.6 & 21.9 \\ 11.7 & 16.6 & 14.9 & 16.2 & 16.3 & 14.6 & 14.9 \\ 6.3 & 7.3 & 7.0 & 7.5 & 7.4 & 8.0 & 7.1 \\ 2.1 & 3.1 & 0.7 & 3.5 & 3.8 & 4.4 & 4.9 \\ 0.6 & 0.2 & -0.2 & 1.1 & 0.5 & 1.5 & 1.8 \\ 1.5 & 2.8 & 0.9 & 2.5 & 3.3 & 2.9 & 3.0 \\ -15.9 & -20.8 & -21.2 & -20.2 & -19.8 & -18.2 & -17.1 \\ & & & & & & \\ 16.0 & 16.9 & 15.8 & 17.4 & 16.9 & 17.4 & 17.9 \\ 28.2 & 35.1 & 32.4 & 34.5 & 34.4 & 32.1 & 32.6 \\ -13.1 & -18.3 & -16.5 & -17.0 & -17.5 & -14.7 & -14.7 \\ 9.3 & 12.8 & 12.0 & 11.9 & 12.1 & 10.1 & 10.5 \\ -3.8 & -5.5 & -4.5 & -5.1 & -5.4 & -4.6 & -4.2 \\ -3.4 & -3.2 & -3.6 & -3.1 & -4.0 & -2.9 & -2.6 \\ 3.6 & 6.0 & 5.6 & 5.2 & 4.3 & 4.6 & 4.4 \\ 0.2 & -0.5 & -1.1 & -0.1 & 0.7 & 0.0 & -0.2\end{array}$

External current account, before grants

Overall balance of payments

Net international reserves (end of period)

Gross international reserves (end of period)

In months of imports of goods and nonfactor services

In months of imports of goods and nonfactor services,

excl. megaprojects

In months of imports of goods and nonfactorservices,

excl. megaprojects and foreign financed capital imports

$-1,285$
279
1,504

1,504

1,520

5.0

6.4
(Millions of U.S. dollars, unless otherwise indicated)

$\begin{array}{rr}-1,954 & -2,058 \\ 81 & 121 \\ 1,589 & 1,625 \\ 1,605 & 1,641 \\ 4.6 & 4.6 \\ 5.7 & 5.8 \\ & \\ 7.9 & 7.7\end{array}$

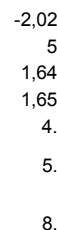

\section{$-2,107$
-17}

1,608

1,625

4.5

5.6

8.1 $\begin{array}{rr}-2,092 & -2,134 \\ 58 & 72\end{array}$

$1,666 \quad 1,739$

$1,682 \quad 1,754$

$4.5 \quad 4.6$

$\begin{array}{ll}5.8 & 5.9\end{array}$

$8.2 \quad 8.2$

Sources: Mozambican authorities; and IMF staff estimates and projections.

${ }^{1}$ Minus sign indicates depreciation.

${ }^{2}$ Includes the issuance of government securities for the central bank recapitalization in years 2005-07.

${ }^{3}$ Includes movement in the government account set abroad with the proceed of the Moatize coal mine concession.

${ }^{4}$ Data from 2008 onwards based on data following technical revisions to monetary survey finalized in January 2008

${ }^{5}$ Includes World Bank disbursement of 2009 budget support in 2008. 
Table 2. Mozambique: Government Finances, 2007-11

\begin{tabular}{|c|c|c|c|c|c|c|c|}
\hline & \multirow{2}{*}{$\begin{array}{r}2007 \\
\text { Act. }\end{array}$} & \multicolumn{2}{|l|}{2008} & \multicolumn{2}{|l|}{2009} & \multirow{2}{*}{$\begin{array}{c}2010 \\
\text { Proj. }\end{array}$} & \multirow{2}{*}{$\begin{array}{l}2011 \\
\text { Proj }\end{array}$} \\
\hline & & $\begin{array}{c}\text { Country Report } \\
08 / 220\end{array}$ & Proj. & $\begin{array}{c}\text { Country Report } \\
08 / 220\end{array}$ & Proj. & & \\
\hline & \multicolumn{7}{|c|}{ (Billions of MT) } \\
\hline Total revenue & 33.06 & 38.24 & 37.20 & 44.53 & 44.98 & 52.80 & 60.72 \\
\hline Tax revenue & 29.08 & 32.69 & 32.57 & 37.83 & 39.06 & 46.49 & 53.42 \\
\hline Taxes on income and profits & 9.28 & 10.00 & 11.10 & 12.80 & 12.11 & 17.83 & 20.51 \\
\hline Taxes on goods and services & 15.51 & 18.89 & 17.28 & 21.06 & 22.63 & 24.62 & 28.66 \\
\hline Of which: on petroleum products & 2.26 & 3.16 & 2.54 & 3.40 & 3.59 & 3.95 & 4.54 \\
\hline Taxes on international trade & 3.78 & 3.12 & 3.53 & 3.20 & 3.51 & 3.16 & 3.24 \\
\hline Other taxes & 0.52 & 0.68 & 0.66 & 0.77 & 0.81 & 0.87 & 1.00 \\
\hline Nontax revenue & 3.98 & 5.54 & 4.64 & 6.70 & 5.93 & 6.32 & 7.31 \\
\hline Total expenditure and net lending & 58.45 & 79.71 & 76.04 & 88.04 & 91.61 & 97.08 & 109.91 \\
\hline Current expenditure & 31.84 & 37.72 & 37.72 & 41.77 & 43.59 & 48.01 & 54.00 \\
\hline Compensation to employees & 16.09 & 18.82 & 18.86 & 20.59 & 22.46 & 24.19 & 26.47 \\
\hline Goods and services & 8.34 & 10.03 & 9.93 & 10.48 & 11.56 & 12.30 & 13.97 \\
\hline Interest on public debt & 1.28 & 1.61 & 1.31 & 2.15 & 1.39 & 1.61 & 1.90 \\
\hline Domestic & 0.87 & 1.12 & 0.82 & 1.48 & 0.85 & 0.90 & 0.88 \\
\hline External & 0.40 & 0.48 & 0.48 & 0.67 & 0.54 & 0.72 & 1.02 \\
\hline Transfer payments & 6.13 & 7.27 & 7.62 & 8.56 & 8.18 & 9.90 & 11.65 \\
\hline Domestic current primary balance & 2.50 & 2.12 & 0.79 & 4.90 & 2.78 & 6.41 & 8.63 \\
\hline Capital expenditure & 24.31 & 37.54 & 35.01 & 41.44 & 43.32 & 44.30 & 50.44 \\
\hline Grant finance projects & 6.64 & 11.76 & 11.84 & 12.41 & 13.89 & 12.60 & 14.26 \\
\hline Projet loans & 3.98 & 6.50 & 5.35 & 6.86 & 7.21 & 7.60 & 7.87 \\
\hline Locally financed & 7.94 & 9.61 & 9.51 & 13.01 & 13.45 & 15.62 & 17.78 \\
\hline Grant-financed special programs & 3.70 & 7.89 & 6.55 & 8.27 & 7.54 & 7.17 & 9.50 \\
\hline Direct financing & 0.85 & 0.85 & 0.85 & 0.89 & 1.24 & 1.31 & 1.02 \\
\hline Expenditure financed with fund for the concession mine of Tete & 1.20 & 0.92 & 0.92 & 0.00 & 0.00 & 0.00 & 0.00 \\
\hline Net lending & 2.30 & 4.45 & 3.31 & 4.83 & 4.70 & 4.78 & 5.48 \\
\hline Lending to Public Enterprises & 2.48 & 4.63 & 3.48 & 5.03 & 4.74 & 5.01 & 5.73 \\
\hline Locally financed & -0.18 & -0.18 & -0.17 & -0.20 & -0.05 & -0.23 & -0.26 \\
\hline Domestic primary balance, before grants, above the line ${ }^{1}$ & -5.27 & -7.32 & -8.56 & -7.90 & -10.62 & -8.98 & -8.89 \\
\hline Unallocated revenue $(+) /$ expenditure $(-)^{2}$ & -1.78 & 0.00 & 0.00 & 0.00 & 0.00 & 0.00 & 0.00 \\
\hline Overall balance, before grants (below the line) & -27.16 & -41.47 & -38.84 & -43.51 & -46.63 & -44.28 & -49.18 \\
\hline Grants received & 19.27 & 28.97 & 28.16 & 30.49 & 32.21 & 30.48 & 35.52 \\
\hline Project & 11.19 & 20.50 & 19.23 & 21.58 & 22.66 & 21.08 & 24.78 \\
\hline Investment Projects & 6.64 & 11.76 & 11.84 & 12.41 & 13.89 & 12.60 & 14.26 \\
\hline Special programs & 3.70 & 7.89 & 6.55 & 8.27 & 7.54 & 7.17 & 9.50 \\
\hline Direct financing & 0.85 & 0.85 & 0.85 & 0.89 & 1.24 & 1.31 & 1.02 \\
\hline Nonproject & 8.08 & 8.47 & 8.92 & 8.91 & 9.55 & 9.40 & 10.74 \\
\hline Overall balance, after grants & -7.90 & -12.51 & -10.69 & -13.03 & -14.42 & -13.80 & -13.66 \\
\hline Central bank transfer of HIPC Initiative assistance by the IMF & 0.04 & 0.00 & 0.00 & 0.00 & 0.00 & 0.00 & 0.00 \\
\hline Net external financing & 7.47 & 13.59 & 13.26 & 13.21 & 11.55 & 14.05 & 13.86 \\
\hline Disbursements & 8.29 & 13.58 & 13.23 & 14.48 & 12.74 & 15.66 & 15.68 \\
\hline Project & 3.98 & 6.50 & 5.35 & 6.86 & 7.21 & 7.60 & 7.80 \\
\hline Nonproject & 4.31 & 7.09 & 7.88 & 7.62 & 5.53 & 8.06 & 7.88 \\
\hline Budget Support & 2.59 & 2.46 & 4.41 & 2.59 & 0.78 & 3.05 & 3.00 \\
\hline Loan to Public Enterprises & 1.72 & 4.63 & 3.48 & 5.03 & 4.74 & 5.01 & 4.88 \\
\hline Cash amortization & -0.82 & -0.92 & -0.90 & -1.27 & -1.19 & -1.61 & -1.82 \\
\hline Investment abroad ${ }^{3}$ & 0.00 & 0.92 & 0.92 & 0.00 & 0.00 & 0.00 & 0.00 \\
\hline Net domestic financing ${ }^{4}$ & 0.38 & -1.20 & -2.69 & -0.18 & 1.82 & -0.25 & -0.20 \\
\hline Adjusted for World Bank advanced budget support ${ }^{5}$ & 0.38 & -1.20 & -0.73 & -0.18 & -0.14 & -0.25 & -0.20 \\
\hline Privatization & 0.00 & 0.12 & 0.12 & 0.00 & 1.04 & 0.00 & 0.00 \\
\hline \multicolumn{8}{|l|}{ Memorandum items: } \\
\hline Domestic primary balance, before grants, below the line ${ }^{1}$ & -7.04 & -7.32 & -8.56 & -7.90 & -10.62 & -8.98 & -8.89 \\
\hline Grants and loan disbursements & 27.56 & 42.55 & 41.38 & 44.97 & 44.95 & 46.14 & 51.20 \\
\hline Bonds issuance for strengthening the central bank balance sheet & 1.50 & 0.00 & 0.00 & 0.00 & 0.00 & 0.00 & 0.00 \\
\hline Nominal GDP & 207.14 & 226.79 & 234.94 & 255.33 & 266.48 & 303.09 & 339.25 \\
\hline
\end{tabular}

Sources: Mozambican authorities; and IMF staff estimates and projections.

${ }_{1}^{1}$ Revenue minus noninterest current expenditure minus locally financed capital expenditure and locally financed net lending. Unallocated revenue and expenditure are included in the primary balance.

${ }^{2}$ Residual discrepancy between identified sources and use of funds.

${ }^{3}$ Tracks the movements in the government account set up abroad with the proceeds of the Moatize coal mine concession.

${ }^{4}$ Excludes recapitilization bonds issued to the Bank of Mozambique.

${ }^{5} \mathrm{~A}$ World Bank disbursement of US $\$ 80$ million budget support designated for 2009 was advanced to 2008 . 
Table 2. Mozambique: Government Finances, 2007-11 (cont.)

\begin{tabular}{|c|c|c|c|c|c|c|c|c|}
\hline & \multirow{2}{*}{$\begin{array}{r}2007 \\
\text { Act. }\end{array}$} & \multicolumn{3}{|c|}{2008} & \multicolumn{2}{|l|}{2009} & \multirow{2}{*}{$\begin{array}{l}2010 \\
\text { Proj. }\end{array}$} & \multirow{2}{*}{$\begin{array}{l}2011 \\
\text { Proj. }\end{array}$} \\
\hline & & $\begin{array}{c}\text { Country Report } \\
08 / 220\end{array}$ & Proj. ${ }^{6}$ & Proj. & $\begin{array}{c}\text { Country Report } \\
08 / 220\end{array}$ & Proj. & & \\
\hline & \multicolumn{8}{|c|}{ (Percent of GDP, unless otherwise specified) } \\
\hline Total revenue & 16.0 & 16.9 & 16.4 & 15.8 & 17.4 & 16.9 & 17.4 & 17.9 \\
\hline Tax revenue & 14.0 & 14.4 & 14.4 & 13.9 & 14.8 & 14.7 & 15.3 & 15.7 \\
\hline Taxes on income and profits & 4.5 & 4.4 & 4.9 & 4.7 & 5.0 & 4.5 & 5.9 & 6.0 \\
\hline Taxes on goods and services & 7.5 & 8.3 & 7.6 & 7.4 & 8.2 & 8.5 & 8.1 & 8.4 \\
\hline Of which: on petroleum products & 1.1 & 1.4 & 1.1 & 1.1 & 1.3 & 1.3 & 1.3 & 1.3 \\
\hline Taxes on international trade & 1.8 & 1.4 & 1.6 & 1.5 & 1.3 & 1.3 & 1.0 & 1.0 \\
\hline Other taxes & 0.2 & 0.3 & 0.3 & 0.3 & 0.3 & 0.3 & 0.3 & 0.3 \\
\hline Nontax revenue & 1.9 & 2.4 & 2.0 & 2.0 & 2.6 & 2.2 & 2.1 & 2.2 \\
\hline Total expenditure and net lending & 28.2 & 35.1 & 33.5 & 32.4 & 34.5 & 34.4 & 32.0 & 32.4 \\
\hline Current expenditure & 15.4 & 16.6 & 16.6 & 16.1 & 16.4 & 16.4 & 15.8 & 15.9 \\
\hline Compensation to employees & 7.8 & 8.3 & 8.3 & 8.0 & 8.1 & 8.4 & 8.0 & 7.8 \\
\hline Goods and services & 4.0 & 4.4 & 4.4 & 4.2 & 4.1 & 4.3 & 4.1 & 4.1 \\
\hline Interest on public debt & 0.6 & 0.7 & 0.6 & 0.6 & 0.8 & 0.5 & 0.5 & 0.6 \\
\hline Domestic & 0.4 & 0.5 & 0.4 & 0.3 & 0.6 & 0.3 & 0.3 & 0.3 \\
\hline External & 0.2 & 0.2 & 0.2 & 0.2 & 0.3 & 0.2 & 0.2 & 0.3 \\
\hline Transfer payments & 3.0 & 3.2 & 3.4 & 3.2 & 3.4 & 3.1 & 3.3 & 3.4 \\
\hline Domestic current primary balance & 1.2 & 0.9 & 0.3 & 0.3 & 1.9 & 1.0 & 2.1 & 2.5 \\
\hline Capital expenditure & 11.7 & 16.6 & 15.4 & 14.9 & 16.2 & 16.3 & 14.6 & 14.9 \\
\hline Grant finance projects & 3.2 & 5.2 & 5.2 & 5.0 & 4.9 & 5.2 & 4.2 & 4.2 \\
\hline Projet loans & 1.9 & 2.9 & 2.4 & 2.3 & 2.7 & 2.7 & 2.5 & 2.3 \\
\hline Locally financed & 3.8 & 4.2 & 4.2 & 4.0 & 5.1 & 5.0 & 5.2 & 5.2 \\
\hline Grant-financed special programs & 1.8 & 3.5 & 2.9 & 2.8 & 3.2 & 2.8 & 2.4 & 2.8 \\
\hline Direct financing & 0.4 & 0.4 & 0.4 & 0.4 & 0.3 & 0.5 & 0.4 & 0.3 \\
\hline Expenditure financed with fund for the concession mine of Tete & 0.6 & 0.4 & 0.4 & 0.4 & 0.0 & 0.0 & 0.0 & 0.0 \\
\hline Net lending & 1.1 & 2.0 & 1.5 & 1.4 & 1.9 & 1.8 & 1.6 & 1.6 \\
\hline Lending to Public Enterprises & 1.2 & 2.0 & 1.5 & 1.5 & 2.0 & 1.8 & 1.7 & 1.7 \\
\hline Locally financed & -0.1 & -0.1 & -0.1 & -0.1 & -0.1 & 0.0 & -0.1 & -0.1 \\
\hline Domestic primary balance, before grants, above the line ${ }^{1}$ & -2.5 & -3.2 & -3.8 & -3.6 & -3.1 & -4.0 & -3.0 & -2.6 \\
\hline Unallocated revenue $(+) /$ expenditure $(-)^{2}$ & -0.9 & 0.0 & 0.0 & 0.0 & 0.0 & 0.0 & 0.0 & 0.0 \\
\hline Overall balance, before grants (below the line) & -13.1 & -18.3 & -17.1 & -16.5 & -17.0 & -17.5 & -14.6 & -14.5 \\
\hline Grants received & 9.3 & 12.8 & 12.4 & 12.0 & 11.9 & 12.1 & 10.1 & 10.5 \\
\hline Project & 5.4 & 9.0 & 8.5 & 8.2 & 8.5 & 8.5 & 7.0 & 7.3 \\
\hline Investment Projects & 3.2 & 5.2 & 5.2 & 5.0 & 4.9 & 5.2 & 4.2 & 4.2 \\
\hline Special programs & 1.8 & 3.5 & 2.9 & 2.8 & 3.2 & 2.8 & 2.4 & 2.8 \\
\hline Direct financing & 0.4 & 0.4 & 0.4 & 0.4 & 0.3 & 0.5 & 0.4 & 0.3 \\
\hline Nonproject & 3.9 & 3.7 & 3.9 & 3.8 & 3.5 & 3.6 & 3.1 & 3.2 \\
\hline Overall balance, after grants & -3.8 & -5.5 & -4.7 & -4.5 & -5.1 & -5.4 & -4.6 & -4.0 \\
\hline Central bank transfer of HIPC Initiative assistance by the IMF & 0.0 & 0.0 & 0.0 & 0.0 & 0.0 & 0.0 & 0.0 & 0.0 \\
\hline Net external financing & 3.6 & 6.0 & 5.8 & 5.6 & 5.2 & 4.3 & 4.6 & 4.1 \\
\hline Disbursements & 4.0 & 6.0 & 5.8 & 5.6 & 5.7 & 4.8 & 5.2 & 4.6 \\
\hline Project & 1.9 & 2.9 & 2.4 & 2.3 & 2.7 & 2.7 & 2.5 & 2.3 \\
\hline Nonproject & 2.1 & 3.1 & 3.5 & 3.4 & 3.0 & 2.1 & 2.7 & 2.3 \\
\hline Budget Support & 1.3 & 1.1 & 1.9 & 1.9 & 1.0 & 0.3 & 1.0 & 0.9 \\
\hline Loan to Public Enterprises & 0.8 & 2.0 & 1.5 & 1.5 & 2.0 & 1.8 & 1.7 & 1.4 \\
\hline Cash amortization & -0.4 & -0.4 & -0.4 & -0.4 & -0.5 & -0.4 & -0.5 & -0.5 \\
\hline Investment abroad ${ }^{3}$ & 0.0 & 0.4 & 0.4 & 0.4 & 0.0 & 0.0 & 0.0 & 0.0 \\
\hline Net domestic financing ${ }^{4}$ & 0.2 & -0.5 & -1.2 & -1.1 & -0.1 & 0.7 & -0.1 & -0.1 \\
\hline Adjusted for World Bank advanced budget support ${ }^{5}$ & 0.2 & -0.5 & -0.3 & -0.3 & -0.1 & -0.1 & -0.1 & -0.1 \\
\hline Privatization & 0.0 & 0.1 & 0.1 & 0.1 & 0.0 & 0.4 & 0.0 & 0.0 \\
\hline \multicolumn{9}{|l|}{ Memorandum items: } \\
\hline Domestic primary balance, before grants, below the line ${ }^{1}$ & -3.4 & -3.2 & -3.8 & -3.6 & -3.1 & -4.0 & -3.0 & -2.6 \\
\hline Grants and loan disbursements & 13.3 & 18.8 & 18.2 & 17.6 & 17.6 & 16.9 & 15.2 & 15.1 \\
\hline Bonds issuance for strengthening the central bank balance sheet & 0.7 & 0.0 & 0.0 & 0.0 & 0.0 & 0.0 & 0.0 & 0.0 \\
\hline Nominal GDP (billion of MT) & 207.1 & 226.8 & 226.8 & 234.9 & 255.3 & 266.5 & 303.1 & 339.3 \\
\hline
\end{tabular}

Sources: Mozambican authorities; and IMF staff estimates and projections.

${ }^{1}$ Revenue minus noninterest current expenditure minus locally financed capital expenditure and locally financed net lending. Unallocated revenue and expenditure are included in the primary balance.

${ }^{2}$ Residual discrepancy between identified sources and use of funds.

${ }^{3}$ Tracks the movements in the government account set up abroad with the proceeds of the Moatize coal mine concession.

${ }^{4}$ Excludes recapitilization bonds issued to the Bank of Mozambique.

${ }^{5}$ A World Bank disbursement of US $\$ 80$ million budget support designated for 2009 was advanced to 2008 .

${ }^{6}$ Percent of programmed nominal GDP. 
Table 3. Mozambique: Monetary Survey, 2007-091

(Billions of MT, unless otherwise stated)

\begin{tabular}{|c|c|c|c|c|c|}
\hline & 2007 & 2008 & & 2009 & \\
\hline & Act. & $\begin{array}{c}\text { Country Report } \\
08 / 220\end{array}$ & Proj. & $\begin{array}{c}\text { Country Report } \\
08 / 220\end{array}$ & Proj. \\
\hline Central bank & & & & & \\
\hline $\begin{array}{l}\text { Net foreign assets } \\
\text { (billions of U.S. dollars) } \\
\text { Net international reserves } \\
\text { (billions of U.S. dollars) } \\
\text { Net domestic assets } \\
\text { Credit to government (net) }{ }^{2} \\
\text { Credit to banks (net) } \\
\text { Credit to the economy } \\
\text { Other items (net; assets +) } \\
\text { Reserve money } \\
\text { Currency in circulation } \\
\text { Bank Deposits (reserves) in BM } \\
\text { Required reserves } \\
\text { Free reserves }\end{array}$ & $\begin{array}{r}36.95 \\
1.55 \\
35.81 \\
1.50 \\
-19.13 \\
-18.95 \\
0.09 \\
0.31 \\
-0.57 \\
17.82 \\
10.94 \\
6.88 \\
6.44 \\
0.44\end{array}$ & $\begin{array}{r}42.15 \\
1.64 \\
40.81 \\
1.59 \\
-23.22 \\
-19.60 \\
0.09 \\
0.31 \\
-4.02 \\
18.93 \\
11.66 \\
7.28 \\
6.88 \\
0.40\end{array}$ & $\begin{array}{r}40.88 \\
1.67 \\
39.84 \\
1.62 \\
-21.26 \\
-20.59 \\
0.26 \\
0.34 \\
-1.27 \\
19.62 \\
12.04 \\
7.59 \\
7.27 \\
0.31\end{array}$ & $\begin{array}{r}44.45 \\
1.69 \\
43.07 \\
1.64 \\
-23.24 \\
-18.71 \\
0.11 \\
0.31 \\
-4.94 \\
21.21 \\
12.97 \\
8.24 \\
7.84 \\
0.40\end{array}$ & $\begin{array}{r}43.77 \\
1.65 \\
42.65 \\
1.61 \\
-21.99 \\
-17.67 \\
0.25 \\
0.34 \\
-4.90 \\
21.79 \\
13.00 \\
8.79 \\
8.47 \\
0.31\end{array}$ \\
\hline Deposit money banks & & & & & \\
\hline $\begin{array}{l}\text { Net foreign assets } \\
\text { (billions of U.S. dollars) } \\
\text { Net domestic assets } \\
\text { Banks' reserves } \\
\text { Credit to central bank (net) } \\
\text { Credit to government (net) } \\
\text { Credit to the economy } \\
\text { Of which: in foreign currency } \\
\text { (billions of U.S. dollars) } \\
\text { Other items (net; assets }+ \text { ) } \\
\text { Deposits } \\
\text { Demand and savings deposits } \\
\text { Time deposits }\end{array}$ & $\begin{array}{r}17.33 \\
0.73 \\
40.21 \\
8.75 \\
-0.01 \\
8.97 \\
30.57 \\
7.53 \\
0.32 \\
-8.07 \\
57.55 \\
36.81 \\
18.62\end{array}$ & $\begin{array}{r}22.73 \\
0.89 \\
46.08 \\
9.27 \\
-0.09 \\
7.60 \\
38.71 \\
7.74 \\
0.30 \\
-9.41 \\
68.82 \\
45.80 \\
23.01\end{array}$ & $\begin{array}{r}21.21 \\
0.86 \\
49.14 \\
7.59 \\
-0.26 \\
8.23 \\
43.73 \\
10.93 \\
0.45 \\
-10.15 \\
70.35 \\
46.68 \\
23.66\end{array}$ & $\begin{array}{r}23.22 \\
0.89 \\
55.25 \\
9.80 \\
-0.11 \\
6.53 \\
48.94 \\
8.56 \\
0.33 \\
-9.90 \\
78.47 \\
52.15 \\
26.33\end{array}$ & $\begin{array}{r}24.46 \\
0.92 \\
57.52 \\
8.79 \\
-0.25 \\
7.14 \\
54.21 \\
12.20 \\
0.46 \\
-12.37 \\
81.98 \\
54.53 \\
27.45\end{array}$ \\
\hline Monetary survey & & & & & \\
\hline $\begin{array}{l}\text { Net foreign assets } \\
\text { (billions of U.S. dollars) } \\
\text { Net domestic assets } \\
\text { Domestic credit } \\
\text { Credit to government (net) } \\
\text { Credit to the economy } \\
\text { Of which: in foreign currency } \\
\text { Of which: in domestic currency } \\
\text { Other items (net; assets +) } \\
\text { Money and quasi money (M3) } \\
\text { Foreign currency deposits } \\
\text { (billions of U.S. dollars) } \\
\text { M2 Currency outside banks } \\
\text { Domestic currency deposits }\end{array}$ & $\begin{array}{r}54.28 \\
2.28 \\
12.82 \\
20.89 \\
-9.99 \\
30.88 \\
7.53 \\
23.35 \\
-8.08 \\
67.10 \\
24.33 \\
1.02 \\
42.77 \\
8.95 \\
33.82\end{array}$ & $\begin{array}{r}64.89 \\
2.53 \\
13.59 \\
27.03 \\
-11.99 \\
39.02 \\
7.80 \\
31.21 \\
-13.44 \\
78.48 \\
28.94 \\
1.13 \\
49.54 \\
9.66 \\
39.88\end{array}$ & $\begin{array}{r}62.08 \\
2.53 \\
18.60 \\
30.02 \\
-12.36 \\
42.38 \\
10.59 \\
31.78 \\
-11.42 \\
80.68 \\
28.47 \\
1.16 \\
52.21 \\
10.34 \\
41.87\end{array}$ & $\begin{array}{r}67.67 \\
2.58 \\
22.22 \\
37.06 \\
-12.19 \\
49.25 \\
8.62 \\
40.63 \\
-14.84 \\
89.89 \\
33.15 \\
1.26 \\
56.74 \\
11.42 \\
45.32\end{array}$ & $\begin{array}{r}68.23 \\
2.57 \\
25.47 \\
42.75 \\
-10.53 \\
53.28 \\
11.99 \\
41.29 \\
-17.27 \\
93.71 \\
33.25 \\
1.25 \\
60.46 \\
11.73 \\
48.73\end{array}$ \\
\hline Memorandum items: & & & & & \\
\hline $\begin{array}{l}\text { Credit to government (net) Prog. } \\
\text { Credit to government (net flows) Prog. }\end{array}$ & $\begin{array}{r}-10.68 \\
-0.71\end{array}$ & $\begin{array}{r}-11.62 \\
-1.20\end{array}$ & $\begin{array}{r}-13.37 \\
-2.69\end{array}$ & $\begin{array}{r}-11.80 \\
-0.18\end{array}$ & $\begin{array}{r}-11.55 \\
1.82\end{array}$ \\
\hline $\begin{array}{l}\text { (percent change of beginning period M3) } \\
\text { Net foreign assets } \\
\text { Net domestic assets } \\
\text { Credit to government (net) } \\
\text { Credit to the economy } \\
\text { Other items (net; asset +) }\end{array}$ & $\begin{array}{r}12.3 \\
11.8 \\
3.9 \\
8.1 \\
-0.3\end{array}$ & $\begin{array}{r}14.8 \\
2.2 \\
-3.1 \\
12.1 \\
-6.9\end{array}$ & $\begin{array}{r}11.6 \\
8.6 \\
-3.5 \\
17.1 \\
-5.0\end{array}$ & $\begin{array}{r}3.5 \\
11.0 \\
-0.3 \\
13.0 \\
-1.8\end{array}$ & $\begin{array}{r}7.6 \\
8.5 \\
2.3 \\
13.5 \\
-7.3\end{array}$ \\
\hline $\begin{array}{l}\text { Reserve money (percent change) } \\
\text { M2 (percent change) } \\
\text { M3 (percent change) } \\
\text { M2 (percent of GDP) } \\
\text { M3 (percent of GDP) }\end{array}$ & $\begin{array}{l}20.9 \\
21.6 \\
24.1 \\
20.6 \\
32.4\end{array}$ & $\begin{array}{r}6.2 \\
15.8 \\
17.0 \\
21.8 \\
34.6\end{array}$ & $\begin{array}{l}10.1 \\
22.1 \\
20.2 \\
22.2 \\
34.3\end{array}$ & $\begin{array}{l}12.0 \\
14.5 \\
14.5 \\
22.2 \\
35.2\end{array}$ & $\begin{array}{l}11.0 \\
15.8 \\
16.1 \\
22.7 \\
35.2\end{array}$ \\
\hline $\begin{array}{l}\text { Credit to the economy (percent change) } \\
\text { Credit to the economy (percent of GDP) } \\
\text { Currency outside banks (percent of M3) } \\
\text { Foreign currency deposits (percent of M3) }\end{array}$ & $\begin{array}{l}16.6 \\
14.9 \\
13.3 \\
36.3\end{array}$ & $\begin{array}{l}26.4 \\
17.2 \\
12.3 \\
36.9\end{array}$ & $\begin{array}{l}37.2 \\
18.0 \\
12.8 \\
35.3\end{array}$ & $\begin{array}{l}26.2 \\
19.3 \\
12.7 \\
36.9\end{array}$ & $\begin{array}{l}25.7 \\
20.0 \\
12.5 \\
35.5\end{array}$ \\
\hline $\begin{array}{l}\text { Money multiplier (M2/reserve money) } \\
\text { Money multiplier (M3/reserve money) } \\
\text { Velocity (GDP/M2) } \\
\text { Velocity (GDP/M3) }\end{array}$ & $\begin{array}{l}2.40 \\
3.76 \\
4.84 \\
3.09\end{array}$ & $\begin{array}{l}2.62 \\
4.14 \\
4.58 \\
2.89\end{array}$ & $\begin{array}{l}2.66 \\
4.11 \\
4.50 \\
2.91\end{array}$ & $\begin{array}{l}2.68 \\
4.24 \\
4.50 \\
2.84\end{array}$ & $\begin{array}{l}2.77 \\
4.30 \\
4.41 \\
2.84\end{array}$ \\
\hline $\begin{array}{l}\text { Nominal GDP } \\
\text { Nominal GDP growth }\end{array}$ & $\begin{array}{r}207 \\
14.9\end{array}$ & $\begin{array}{r}227 \\
12.6\end{array}$ & $\begin{array}{r}235 \\
13.4\end{array}$ & $\begin{array}{r}255 \\
12.6\end{array}$ & $\begin{array}{r}266 \\
13.4\end{array}$ \\
\hline
\end{tabular}

Sources: Bank of Mozambique; and IMF staff estimates and projections.

${ }^{1}$ Following the adoption of a Chart of Accounts in line with the IFRS, the monetary survey was revised back to January 1997 to incorporate more detailed information provided by banks regarding the residency and use of monetary instruments.

${ }^{2}$ Includes MDRI assistance which was transferred to a government blocked deposit account at the BM. The balance is being drawn down

by 0.5 percent of GDP per annum in line with an agreed increase in MDRI-financed government expenditures. 
Table 4. Mozambique: Balance of Payments, 2007-11

(Millions of U.S. dollars, unless otherwise specified)

\begin{tabular}{|c|c|c|c|c|c|c|c|}
\hline & \multirow{2}{*}{$\begin{array}{l}2007 \\
\text { Act. }\end{array}$} & \multicolumn{2}{|l|}{2008} & \multicolumn{2}{|l|}{2009} & \multirow{2}{*}{$\begin{array}{l}2010 \\
\text { Proj. }\end{array}$} & \multirow{2}{*}{$\begin{array}{l}2011 \\
\text { Proj. }\end{array}$} \\
\hline & & $\begin{array}{l}\text { Country Report } \\
08 / 220\end{array}$ & Proj. & $\begin{array}{l}\text { Country Report } \\
08 / 220\end{array}$ & Proj. & & \\
\hline Trade balance & -680 & $-1,052$ & $-1,122$ & $-1,149$ & $-1,483$ & -958 & $-1,009$ \\
\hline Exports, f.o.b. & 2,412 & 2,488 & 2,511 & 2,515 & 2,190 & 2,823 & 2,864 \\
\hline Megaprojects & 1,841 & 1,889 & 1,876 & 1,867 & 1,515 & 2,098 & 2,086 \\
\hline Other exports & 571 & 599 & 635 & 647 & 674 & 726 & 778 \\
\hline Imports, c.i.f. & $-3,092$ & $-3,540$ & $-3,633$ & $-3,664$ & $-3,673$ & $-3,781$ & $-3,872$ \\
\hline Megaprojects & -689 & -693 & -738 & -764 & -731 & -816 & -838 \\
\hline Other imports & $-2,403$ & $-2,847$ & $-2,895$ & $-2,900$ & $-2,942$ & $-2,965$ & $-3,035$ \\
\hline Services and incomes (net) & -605 & -901 & -936 & -876 & -624 & $-1,135$ & $-1,126$ \\
\hline Receipts & 684 & 693 & 706 & 710 & 724 & 742 & 760 \\
\hline Expenditures & $-1,289$ & $-1,594$ & $-1,642$ & $-1,586$ & $-1,349$ & $-1,877$ & $-1,886$ \\
\hline Of which: interest on public debt & -16 & -21 & -19 & -31 & -21 & -42 & -52 \\
\hline Current account, before grants & $-1,285$ & $-1,954$ & $-2,058$ & $-2,025$ & $-2,107$ & $-2,093$ & $-2,135$ \\
\hline Unrequited official transfers & 790 & 1,244 & 1,237 & 1,251 & 1,306 & 1,156 & 1,215 \\
\hline Current account, after grants ${ }^{1}$ & -495 & -710 & -821 & -775 & -802 & -936 & -920 \\
\hline Capital account & 519 & 791 & 805 & 828 & 785 & 993 & 991 \\
\hline Foreign borrowing & 1,464 & 832 & 914 & 954 & 847 & 977 & 986 \\
\hline Public (including IMF) & 325 & 563 & 539 & 569 & 508 & 578 & 564 \\
\hline Private $^{2}$ & 1,139 & 269 & 375 & 386 & 340 & 398 & 422 \\
\hline Amortization & $-1,140$ & -406 & -427 & -447 & -476 & -485 & -530 \\
\hline Public & -32 & -16 & -37 & -18 & -47 & -20 & -25 \\
\hline Private $^{3}$ & $-1,108$ & -390 & -390 & -429 & -429 & -466 & -505 \\
\hline Direct investment (net) ${ }^{4}$ & 427 & 457 & 455 & 489 & 471 & 501 & 534 \\
\hline Change in commercial bank NFA & -203 & -108 & -137 & 0 & -57 & 0 & 0 \\
\hline Short-term capital and errors and omissions (net) & 255 & 0 & 137 & 0 & 0 & 0 & 0 \\
\hline Overall balance & 279 & 81 & 121 & 53 & -17 & 57 & 71 \\
\hline Financing & -279 & -81 & -121 & -53 & 17 & -57 & -71 \\
\hline $\begin{array}{l}\text { Of which: Bank of Mozambique } \\
\text { gross reserve assets (increase -) }\end{array}$ & \multicolumn{6}{|c|}{ Of which: Bank of Mozambique } & -71 \\
\hline Financing gap & 0 & 0 & 0 & 0 & 0 & 0 & 0 \\
\hline \multicolumn{8}{|l|}{ Memorandum items: } \\
\hline Use of Fund credit (net) & 2 & 0 & 0 & 0 & 0 & 0 & -2 \\
\hline \multicolumn{8}{|l|}{ Current account deficit (percent of GDP) } \\
\hline Before grants & 15.9 & 20.8 & 21.2 & 20.2 & 19.8 & 18.2 & 17.1 \\
\hline After grants & 6.1 & 7.6 & 8.4 & 7.7 & 7.5 & 8.2 & 7.4 \\
\hline Net international reserves ${ }^{5}$ & 1,504 & 1589 & 1,625 & 1,642 & 1,608 & 1,665 & 1,738 \\
\hline Gross international reserves & 1,520 & 1605 & 1,641 & 1,658 & 1,625 & 1,681 & 1,752 \\
\hline In months of imports of GNFS & 5.0 & 4.6 & 4.6 & 4.6 & 4.5 & 4.5 & 4.6 \\
\hline In months of imports of GNFS, excluding megaprojects & 6.4 & 5.7 & 5.8 & 5.8 & 5.6 & 5.8 & 5.9 \\
\hline $\begin{array}{l}\text { In months of imports of goods and nonfactorservices, excl. } \\
\text { megaprojects and foreign financed capital imports }\end{array}$ & 8.2 & 7.9 & 7.7 & 8.1 & 8.1 & 8.2 & 8.2 \\
\hline
\end{tabular}

Sources: Mozambican authorities; and IMF staff estimates and projections.

${ }^{1}$ Since this presentation still follows the fourth balance of payments manual, MDRI and HIPC grants from the IMF are included in the current account.

${ }^{2}$ Private borrowing, not guaranteed by the government or the Bank of Mozambique. Includes HCB borrowing in 2007.

${ }^{3}$ Includes HCB amortization in 2007.

${ }^{4}$ Projections are underestimated due to lack of information on new megaproject investments.

${ }^{5}$ Based on the new monetary survey from 2008. 


\section{APPENDIX I \\ Republic of Mozambique: Letter of Intent}

October 29, 2008

Mr. Dominique Strauss-Kahn

Managing Director

International Monetary Fund

Washington, D.C. 20431

U.S.A.

Dear Mr. Strauss-Kahn:

1. The Government of Mozambique is implementing a financial and economic program consistent with its medium-term Plano de Acção para a Redução da Pobreza Absoluta II (PARPA II), and with support from the IMF through the Policy Support Instrument (PSI). We have concluded discussions with IMF staff for the third review under the PSI-supported program and our policies under the program for 2009 are summarized in the attached Memorandum of Economic and Financial Policies (MEFP). The technical memorandum of understanding (TMU) defines the terms and conditions of the program.

2. Implementation of the PSI-supported program has been satisfactory. All quantitative and structural assessment criteria (AC) and benchmarks through end-September 2008 were met. The program is broadly on track and we expect to meet the ACs and benchmarks for end-December 2008, except for the AC on reserve money and the end-December benchmark to undertake a study that would serve as a basis for drafting a Public Enterprises Law. As a consequence of the increase in international import prices during 2008, the end-December $\mathrm{AC}$ for reserve money has been modified, as set out in Table 1. The benchmark on the study of Public Enterprises is expected to be met by March 2009. On this basis, we request completion of the third review under the PSI-supported program.

3. The policies set out in the MEFP aim at consolidating macroeconomic stability and sustaining strong broad-based growth through a second wave of reforms towards the achievement of the Millennium Development Goals. Under the program, we have agreed with IMF staff on ACs and benchmarks for 2009. We anticipate that the fourth review on the basis of program performance to end-December 2008 will be conducted by end-June 2009 and that the fifth review on the basis of program performance to end-June 2009 will be conducted by end-December 2009 . 
4. The Government of Mozambique will provide the Fund with such information as the Fund may request in connection with progress in implementing the economic and financial policies and achieving the objectives of the program.

5. The Government of Mozambique believes that the policies set forth in the MEFP are adequate to achieve the objectives of our PSI program; it will take additional measures to that end if deemed necessary. During the implementation of the program, the Government of Mozambique will consult with the Managing Director on the adoption of these measures and in advance of revisions to the policies contained in the PSI, at the initiative of the Government or whenever the Managing Director requests such a consultation.

Sincerely yours,

\author{
/ s / \\ Manuel Chang \\ Minister of Finance
}

\author{
/ s / \\ Ernesto Gouveia Gove \\ Governor \\ Bank of Mozambique
}

Attachments: $\quad$ Memorandum of Economic and Financial Policies

Technical Memorandum of Understanding 


\section{ATtachment I}

\section{Republic of Mozambique: Memorandum of Economic and Financial Policies of the Government of Mozambique for the Third Review of the PSI Supported-Program}

October 29, 2008

1. The Government of Mozambique is committed to further consolidating macroeconomic stability to achieve sustained economic growth and poverty reduction through the pursuit of prudent macroeconomic policies and a second wave of structural reforms. This MEFP reviews the performance under the PSI-supported program with the IMF through September 2008 and describes the policies and targets for 2009.

\section{Program Implementation in $\mathbf{2 0 0 8}$}

\section{A. Background}

2. Mozambique's macroeconomic performance in 2008 has remained strong, although it has been adversely affected by the increase in international food and fuel prices in mid-year. In the 12 months to September, domestic gasoline and food prices rose 30 and 16 percent respectively and headline inflation at the end of 2008 is now expected to be higher than initially projected. Performance was also adversely affected by disruptions to the transmission of electricity via South Africa with consequences for manufacturing and aluminum exports. As a result, projected real economic growth in 2008 has been revised down from 7.0 to 6.5 percent. The current account balance was also adversely affected and, including grants, is expected to deteriorate to 7.9 percent of GDP. This deterioration was mitigated by a decline in the volume of oil imports and more than offset by higher capital inflows, so that the real effective exchange rate appreciated through most of the year and international reserve accumulation by end-2008 is projected to be larger than envisaged.

\section{B. Fiscal Sector}

3. The fiscal strategy during 2008 remained in line with the Government's medium-term strategy to constrain domestic financing to make room for private sector credit. Budget implementation during the year encountered major challenges arising from the sharp increase in fuel and food prices and from difficulties in executing capital expenditures, due to procurement procedures.

4. During the first half of the year, net credit to the Government was better than envisaged under the program, despite a substantial delay of program aid to the second semester. Domestic revenues during the first half of 2008 were stronger than projected, but capital spending fell well short of projections mainly because of procurement constraints. 
5. The Government's fiscal plans were revised in July, in consultation with IMF staff, to reflect measures to mitigate the social impact of higher fuel and food prices. The Government had already introduced an urban transportation subsidy in February which was incorporated into the fiscal program. In June, the Government deferred fuel-related taxes until end-2008, and launched the Food Production Action Plan (FPAP), together costing 1.3 percent of GDP. These additional measures have been largely financed by reductions in non-priority spending and additional World Bank budget support. As a result, the Government's target for net credit to the Government in 2008 has been revised from about 0.5 percent of GDP under the program to about 0.3 percent of GDP (excluding a substantial World Bank disbursement in support of the 2009 budget expected in late 2008).

6. The Revenue Authority (Autoridade Tributária-AT) took steps to improve its operational capacity. With the help of donors, a revised 2009-10 strategy was approved in October. The AT has established indicators of overall performance and a framework for assessing staff performance. Its audit capacity was strengthened through additional hiring, so that the increase in tax audits and coverage was broadly in line with 2008 targets. The AT will develop a strategy on managing and controlling revenues from mega-projects. The implementation of the PDTI - an information technology master plan-will be initiated in early 2009.

7. The study on the stock of VAT arrears due to contractors of large infrastructure projects and covering 80 percent of claims was completed in July. The audit identified MT 1.285 million in arrears, of which MT 200 million is budgeted for clearance in 2009.

8. Several tax policy measures are being introduced to reduce the cost of doing business while increasing the tax base. A bill to simplify taxes for small taxpayers was submitted to Parliament. The law proposes a direct tax on petty trading and small-scale agricultural and industrial activities and aims to reduce tax collection costs and facilitate tax compliance. In October, the adjusted tax benefit code was approved by the Council of Ministers and submitted to Parliament.

9. Public Financial Management (PFM) reforms are progressing well. Most importantly, the second phase of the e-Sistafe reform is being successfully concluded. The sectional classification was completed in May 2008 and the first pilot of the UTRAFE budget initiated. The decree on the multi-currency single treasury account (CUT) was approved in April 2008. On the basis of the civil servants census, the list of e-Sistafe operators will be updated by early 2009. The system for program budgeting was used to prepare the 2009 budgets of 5 ministries, linking their programs to the PARPA sub-programs. Salary payments through eSistafe was initiated in April 2008 in the office of the Inspector General of Finance (IGFInspecção Geral das Finanças), and rolled out to twenty institutions at the national level. The e-Sistafe system was rolled out to a total of twenty State organs at the central level. The census of civil service pensioners was finalized in October 2008. Due to external financing 
constraints, the study of public enterprises will be delayed to 2009 (end-December 2008 structural benchmark).

10. In September 2008, the Council of Ministers approved the medium-term civil service pay policy, which will be implemented as from 2009 in a fiscally sustainable manner. In October 2008 the Government published for the first time the statistical yearbook on civil servants based on the census of civil servants.

11. The MF has continued to restructure the debt management department. The MF has developed strategies aimed at strengthening automated record keeping and analysis. The MF has completed a work plan to implement and strengthen the new departmental structure. It has also reviewed the legislation relating to debt management as the basis for revising the legislation. For 2008, the MF will publish the annual debt management report recently designed by the debt management department.

\section{Monetary Sector}

12. Monetary policy in 2008 has been geared towards price stability. Reserve money expansion to end-June was in line with the program, in an environment of reduced liquidity reserve requirements since April 2008. Implementation of monetary policy in the second quarter was complicated by an unusually large seasonal surge in currency demand. Moreover, as import prices increased beyond program projections, the Bank of Mozambique (BM) has sought to accommodate the pass-through to domestic prices while averting a second-round impact on inflation. As a result, reserve money is now projected to rise 10 percent during the year to end-December 2008. Moreover, headline inflation is expected to be higher than initially programmed at end-2008, inflation excluding food and energy related items will likely remain below 4 percent.

13. The impact of higher import prices on the balance of payments was offset by higher capital inflows so that, until August, the nominal exchange rate versus the US dollar depreciated by around 2 percent and the real effective exchange rate appreciated by 7 percent. Moreover, despite some program aid delays in mid-year, the BM met its external reserve target at both end-June and end-September.

14. The BM has continued to strengthen its monetary and exchange rate policy and operations. To strengthen policy formulation, the BM has stepped up its monitoring, analyzing and forecasting of macroeconomic and monetary indicators. To strengthen operations, the BM introduced repos in July 2008 and will implement measures during the remainder of 2008 to expand their use in place of treasury bills. In June 2008, the Meticalnet was adapted to allow for outright operations for the secondary market (structural benchmark for June 2008). To strengthen liquidity management, the Ministry of Finance (MF) is now providing cash flow projections to the BM on weekly basis. In its foreign exchange operations, the BM has made a sharper delineation between the use of direct bilateral transactions for regular sales of aid inflows and the use of auctions for controlling liquidity. 
15. The BM has implemented measures to strengthen its internal audit. The BM has conducted an external quality assurance review of its internal audit function in accordance with international standards. An internal audit charter has been adopted and the BM has initiated periodic reporting to the BM's Executive Board on the implementation of audit recommendations (end-July structural benchmark). Pending the approval of the new central bank law, the Audit Board has assumed the role of an audit committee. New terms of reference for the audit board were approved, to make it more focused on matters related to accountability, transparency, and internal control and provide oversight over financial reporting, external and internal audits, and systems of internal control (end-June structural AC). The tightening of control over reserve management has experienced some delays: (i) The installation of physical access controls in SWIFT operations is still ongoing and is expected to be completed by end-December 2008; and (ii) the procurement for devices allowing the recording of dealer communications on trading activities, which is expected to be completed by end-December 2008.

\section{Other Structural Policies}

16. To strengthen the management and transparency of mineral resources, in June 2007 the laws no. 11/2007 and no. 12/2007 for specific taxation in the mining and petroleum sectors were approved, respectively. Also in June 2007, the law no. 13/2007, which defines the incentives and benefits applicable on the mining and petroleum sector, was approved. The complementary regulations for both laws (11/2007 and 12/2007) were approved by decrees $\mathrm{n}^{\circ} 4$ and 5/2008. All new agreements in mining and petroleum and gas concession are following the new fiscal legislation and relevant new framework agreements. The MF has started to compile and consolidate data on mega-projects, which is the basis to prepare an annex to the budget execution report. As noted below, the Government intends to accede to the EITI.

17. Mozambique continues its trade liberalization efforts. The SADC free trade area came into effect in January 2008, but problems related rules-of-origin have limited duty-free treatment within SADC. In November 2007, Mozambique, together with other SADC members, reached an interim Economic Partnership Agreement (EPA) with the EU. Members of the SADC EPA will retain quota- and duty-free access to the EU and, on a reciprocal basis, will reduce their customs tariffs on EU imports. Mozambique will implement this agreement from January 2009, subject to the approval by the Parliament. Negotiations with the EU on a final EPA are continuing.

18. The Government has stepped up its efforts to reduce the costs of doing business. The Government approved a decree to consolidate business inspections in selected sectors. A revised draft corporate insolvency law to simplify the business closing process and increase the recovery rate will be re-submitted to the Council of Ministers by end November. The Decree for Simplified Licensing came into force in October, 2008. A proposal to revoke the bank-deposit and eliminate the minimum capital requirements to start up a business was 
submitted to Parliament. A proposal was submitted to the Council of Ministers to introduce the electronic one-stop window, aimed at facilitating cross-border trading. The Council of Ministers approved the creation of an SME Institute. Following the adoption of a competition policy in 2007, a draft competition law and competition authority's statute will be submitted to the Council of Ministers.

19. Progress in judicial reform remains a priority. The Integrated Strategic Plan for the Justice sector was approved by the Council of Ministers in October. The Integrated Strategic Plan will be used as an instrument for strengthening the management and monitoring the capacity of the justice institutions aimed at increasing the provision of legal services to the public. The organic law of the Institute of Judicial Assistance (Instituto de Patrocínio e Assistência Jurídica) was formulated and discussed with national and international partners in October, 2008.

20. The Government is reinvigorating its anti-corruption efforts. After the abolition of the Anti-Corruption Forum in January 2008, the implementation of the Anti-Corruption Strategy has been monitored as part of the public sector reform program by the Public Sector InterMinisterial Committee (CIRESP), chaired by the Prime Minister. In the first semester of 2008, 31 cases were sentenced by the courts for corruption. Furthermore, the Council of Ministers approved in August, 2008 the Report on the implementation of the Anti-Corruption Strategy formulated for the year 2007.

21. Significant progress has been made with respect to improving capacity on producing and disseminating macroeconomic statistics, especially at the INE and the MF. INE has prepared a Statistical Strategic Plan (2008-12), which is integrated with the poverty reduction strategy paper-PARPA II (2006-09) and the MF is increasingly using eSISTAFE to generate public finance statistics not yet in compliance with the 2001 GFS manual.

\section{Outlook and Policies for 2009}

\section{A. Overview}

22. Mozambique's prospects for 2009 and the medium term remain robust. However, there are considerable risks arising from the volatility and uncertainties concerning the impact of the current global financial crisis. Large variations in international prices and the recent substantial price declines for commodities including oil, gas and aluminium have a substantial impact on Mozambique's external trade balance and a deeper slowdown in global demand would also affect export volumes. Moreover, the global financial crisis may adversely affect private capital inflows and the turbulence could spread to the Mozambican financial system. In addition, the economy will remain heavily dependent on inflows from the international donor community for many years to come. Mindful of these risks, the Government will monitor international and domestic developments in these areas closely and stands ready to take actions as needed, in close consultation with the IMF. 
23. On the basis of the current global outlook and notwithstanding the above risks, economic prospects for 2009 remain strong. However, the outlook for real GDP growth has been reduced from 7.0 to 6.7 to accommodate the impact of the exogenous shocks (oil and food prices). The analysis made on the impact of the international financial crisis points to a growth of 6.2 percent of GDP. Lower commodity prices will help headline inflation decline to around 7 percent by the end of 2009. Lower import prices will also help mitigate the impact of lower exports on the current account deficit. However, the weaker global environment could also deter capital investment inflows so that external reserves are expected to decline during the year.

\section{B. Fiscal Sector}

24. In line with the Government's medium-term fiscal strategy, the 2009 budget aims to support economic growth and poverty reduction while limiting domestic financing to provide room for private sector credit growth. The fiscal framework under the program, which is broadly in line with the budget presented to Parliament, envisages a modest net domestic debt repayment of 0.1 percent of GDP (including a substantial World Bank disbursement in support of the 2009 budget expected in late 2008). The Government recognizes there are risks to revenue collection and foreign assistance in the current global economic environment. Should any revenue or financing shortfalls arise, the Government will implement measures to cut nonpriority spending to maintain positive domestic debt repayments.

25. Under the program, domestic revenue is expected to rise from 15.8 percent of GDP in 2008 to 16.9 percent of GDP in 2009. As intended, this incorporates a reversal of the deferral in fuel-related taxes implemented in mid-2008 ( 0.6 percent of GDP). The Government remains committed to raising the domestic tax efforts by 0.5 percent of GDP. This is expected to come mainly from higher excise taxes on tobacco and alcoholic beverages (0.1 percent GDP) implementation of simplified taxes for small taxpayers, implementation of new code of benefits and improvements in revenue administration ( 0.4 percent of GDP). The latter includes more personnel and equipment, including scanner at international entry ports, the collection of tax arrears, and raising the number of taxpayers. Donors have made commitments to provide support around 18 percent of GDP that is similar to 2008 (including the advanced WB disbursement). In addition, the Government expects receipts of about 0.4 percent of GDP from privatization of parastatals.

26. Expenditures are in line with the revised Medium-Term Fiscal Framework (Cenário Fiscal de Médio Prazo-CFMP) and based on donors' aid commitments provided by the donors. Expenditures take into account continued measures to mitigate the impact of high food prices as part of the FPAP (1.2 percent of GDP). The wage bill will rise 0.4 percentage points to reach 8.4 percent of GDP and includes additional hiring of 12,000 teachers and 1,500 health workers. Under the Medium-Term Pay Policy approved in September 2008, the Government is committed to reduce the wage bill back to 8 percent of GDP and below. 
Spending on priority sectors will remain above 65 percent and will be closely monitored. The fiscal framework incorporates election-related costs and of the deferral of the fuel-related taxes introduced in June 2008. Capital expenditures, largely financed by foreign aid, are envisaged to reach 16.3 percent of GDP, 1.4 percentage points higher than in 2008. These scaled-up expenditures would help address infrastructure deficiencies, particularly in the energy sector.

27. The Government will continue to improve the efficiency of the tax system. The AT will implement a revenue payment system through banks. Based on the approved Strategic and Tactical Plans - the Authority will prepare operational plans with specific performance indicators for every department. Furthermore, the AT will improve and simplify the reimbursement system for VAT as well as the payments of personal and corporate income taxes. The authority will elaborate a strategy and action plan to strengthen the audit capacity of the mega-projects unit by end December 2009. The Government is committed to clearing all VAT arrears by 2011, including those already identified in the external audit and will formulate a repayment schedule to this effect.

28. In preparation of the third phase of the e-Sistafe reform, the Government is drafting a PFM vision paper and an action plan for 2009-11, taking into account the PEFA, FiscalROSC and other diagnostic assessments. This will be approved by the MF by end-March 2009 (structural benchmark). The e-Sistafe will be rolled out to additional jurisdictions, bringing the total of districts using e-Sistafe to 42 districts. Salary payments through the eSistafe will be expanded to all central Government ministries, by June 2009. The corporate income tax prototype project in e-Tributação will be implemented by end-September (structural benchmark) with adequate donor funding, and will be expanded to all other taxes during 2011. The MF will publish public domestic and external debt data by end-December 2009. The Multi Currency Treasury Account (CUT) will be available by early 2009 and the Government will encourage donors to transfer project aid through this account.

29. The MF in coordination with the Ministry of Civil Service will prepare an estimate of the new pay policy for the fiscal years 2010 to 2012 by end-August 2009 (structural benchmark).

30. The MF's debt management will be further improved under the FSTAP program by strengthening the institutional capacity of the debt management department, strengthening its procedures and controls, finalizing the consolidation and reconciliation of the debt database for all public debt, and improving the Treasury cash management policies and procedures with assistance of the World Bank. By December, 2009 the MF will start publishing its regular debt sustainability analysis reports.

31. To strengthen monitoring of net lending to public enterprises a draft public enterprises law will be submitted to the Council of Ministers by end-September 2009 
(structural benchmark). It will include the requirement for monthly financial reporting to the $\mathrm{MF}$ and the ring-fencing of commercial activities.

\section{Monetary Sector}

32. The BM is committed to pursuing a prudent monetary policy within the context of a flexible exchange rate regime. Monetary policy will remain geared towards price stability with the objective of reducing inflation to about 7 percent by end-2009. Consistent with this objective, the BM aims to increase reserve money, its operational target, by 11 percent during 2009, which will allow broad money to rise by about 16 percent. Following the large increase in external reserves anticipated in 2008, partly because of the accelerated World Bank disbursements, the BM will target a modest fall in reserves during 2009 to about 4.6 months of imports. In implementing monetary policy, the BM will also continue to rely more heavily on sales of foreign exchange reserves rather than Government securities to sterilize liquidity to make room for credit to the private sector to rise by about 28 percent.

33. The BM will continue refining its monetary policy framework and instruments for implementing policy. To buttress monetary policy formulation and support the decisions of the Monetary Policy Committee, the BM will allocate more resources to monitoring, analyzing and forecasting macroeconomic and monetary indicators. The BM with technical assistance support of the Fund will design a plan to strengthen the monetary policy framework comprising all intermediate steps, including agreement on a core inflation indicator by end-December 2009 (structural benchmark). The BM will also follow up on plans to strengthen policy implementation. In this regard, the BM will continue to focus on strengthening liquidity management, paying particular attention to improving coordination with the MF on its cash flow projections and with banks on their liquidity requirements. To help strengthen the interbank market and liquidity management, the BM will broaden its use of repos to build a flexible and more realistic corridor that better reflects market conditions. To further strengthen the interbank market, the BM will also stimulate the market's use of repos by improving confidence between the market players as result of the increase of volume and frequency of transactions.

34. The BM will implement measures to further buttress its internal audit. In accordance with the audit charter, the internal audit office will report periodically to the BM's Board on the implementation of audit recommendations and on financial developments at the BM. The Government will draft a new central bank law for Mozambique once the SADC central bank model law has been approved (expected in 2010). It will also publish its audited financial statements for 2008 in compliance with IFRS by end-June 2009.

35. Under the strategic plan (2008-10), the BM will continue to strengthen its banking supervision framework by adopting a risk-based approach to supervise financial institutions. The Risk-Based Supervision (RBS) Framework is expected to be finalized by end-2009. A consultant has been appointed under FSTAP to assist BM on the designing of the road map 
for the implementation of Basel II in Mozambique. The consultant will assess the impact of Basel II on financial sector and prepare a transition strategy, procedure manuals, and circulars on procedures.

36. A new foreign exchange law which fully liberalizes current account transactions is currently with Parliament. Following its approval, the Government intends to accept the obligations under Article VIII sections 2, 3, and 4 of the Fund's Articles of Agreement. The recent regulation (Aviso 2/06) pertaining to the current import and export transactions remains suspended.

\section{Other Structural Policies}

37. Following the recent adoption of a new law on social protection, the Government intends to strengthen the social security and supplementary pension system. As part of the restructuring of the social security system, the National Social Security Institute (INSS), the Government has drafted a report on an actuarial study and on a new investment management strategy, that will provide the basis for a plan to reform the INSS into a financially sustainable institution. The Government is finalizing a census of pensioners covered by the civil service pension system managed by the MF and has requested an actuarial study with the World Bank's support. Efforts are also underway to strengthen the regulatory and supervisory framework of the insurance sector and to strengthen the institutional capacity of the Inspecção Geral de Seguros (IGS).

38. To manage the budgetary and macroeconomic implications of megaprojects the Government intends to expand the prerogative of the Institute for National Statistics to collect data on megaprojects so as to develop a comprehensive data system that also satisfies the needs of MF and BM. The Government will strengthen the analytical and forecasting capabilities at the MF in close coordination with other ministries and the BM. The Government will strictly implement the mining and petroleum fiscal regime and related regulations and model contracts to all new projects and continue to adopt nonrecourse financing clauses in future megaprojects.

39. To ensure a transparent management of mineral resources, the Government organized a seminar with representatives of the Parliament, Government, development partners, private sector, and civil society. By June 2009 the Government will formally submit a request to start the process to become a member of the EITI (structural benchmark). The Government, the private sector and civil society will be required to undertake a sequence of actions required for the validation of Mozambique's adherence to the EITI. This process is expected to take at least three years.

40. Based on the strategy approved in February 2008, the Government is accelerating reforms to reduce the costs of doing business. The Government will implement the new corporate insolvency law once approved by the Parliament; implement the one-stop window 
to facilitate trade across borders by December 2009; submit the draft competition law to Parliament; simplify and facilitate electronic tax filing (covered in e-Tributação); amend legislation to accelerate property registration; consolidate progress in streamlining licenses, and establish the SME Institute. Moreover, by end-March 2009, the Ministry of Industry and Trade will issue a decree streamlining the business inspection process (structural benchmark).

41. The Government places particular emphasis on implementing the second phase of the public sector reform program (2006-11), which consists of four pillars: improving service delivery to the citizen and the private sector; strengthening the capacity of local governments, with special emphasis on the Districts (including the decentralization strategy); creating a more professional public sector (including payroll systems, performance evaluation and wage policy); and strengthening good governance systems and the fight against corruption. By end 2009, the Government will draft a Public-Private Partnership Law aimed primarily to avoid, prevent and manage fiscal and para-fiscal risks, which could also be applicable to megaprojects.

42. The Government will prepare a decentralization strategy with the help of the World Bank and other partners. The strategy will propose, among other things, a clear legal, regulatory, and institutional framework for revenue raising and spending responsibilities and functions of sub-national units (provinces, districts, and municipalities), as well as monitoring of their fiscal operations. The strategy will pay due regard to the administrative capacity of sub-national units and need to maintain fiscal control as well as fiscal sustainability in planning, finance, execution and control. A draft decree will be adopted by the Government outlining the general principles of the repayment framework of the OIIL district revolving funds, as well as their management structure.

\section{Program Monitoring}

43. The revised quantitative assessment criteria for end-December 2008 and the new assessment criteria and indicative targets for 2009 are shown in Table 1, with further definitions and explanations contained in the Technical Memorandum of Understanding. Table 3 lists the structural benchmarks for 2009 . 
Table 1. Mozambique: Quantitative Assessment Criteria and Indicative Targets, 2007-09'

\begin{tabular}{|c|c|c|c|c|c|c|c|c|c|c|c|}
\hline & \multicolumn{7}{|c|}{2008} & \multicolumn{4}{|c|}{2009} \\
\hline & \multicolumn{3}{|c|}{$\begin{array}{c}\text { End-June } \\
\text { Assessment } \\
\text { Criteria }\end{array}$} & \multicolumn{2}{|c|}{$\begin{array}{c}\text { End-Sept. } \\
\text { Indicative } \\
\text { Target } \\
\end{array}$} & \multicolumn{2}{|c|}{$\begin{array}{c}\text { End-Dec. } \\
\text { Assessment } \\
\text { Criteria } \\
\end{array}$} & \multirow{2}{*}{$\begin{array}{r}\begin{array}{r}\text { End-March } \\
\text { Indicative } \\
\text { Target }\end{array} \\
\text { Prog. }\end{array}$} & \multirow{2}{*}{$\begin{array}{r}\begin{array}{r}\text { End-June } \\
\text { Assessment } \\
\text { Criteria }\end{array} \\
\text { Prog. } \\
\end{array}$} & \multirow{2}{*}{$\begin{array}{r}\begin{array}{c}\text { End-Sept } \\
\text { Indicative } \\
\text { Target }\end{array} \\
\text { Prog. } \\
\end{array}$} & \multirow{2}{*}{$\begin{array}{r}\begin{array}{r}\text { End-Dec } \\
\text { Indicative } \\
\text { Target }\end{array} \\
\text { Prog. }\end{array}$} \\
\hline & $\begin{array}{c}\text { Country Report } \\
08 / 220\end{array}$ & Adj. & Act. & $\begin{array}{l}\text { Country Report } \\
08 / 220\end{array}$ & Prel. & $\begin{array}{l}\text { Country Report } \\
08 / 220\end{array}$ & Prog. & & & & \\
\hline Net credit to the government (cumulative ceiling) & $-3,210$ & $-2,003$ & $-4,436$ & $-1,332$ & ... & $-1,202$ & $-1,202$ & 133 & $-1,064$ & 446 & 1,825 \\
\hline Stock of reserve money (ceiling) & 17,044 & 17,044 & 17,026 & 17,347 & 17,506 & 18,934 & 19,622 & 17,622 & 19,333 & 19,544 & 21,788 \\
\hline Stock of net international reserves of the BM (floor, in millions of U.S. dollars) & 1,704 & 1,604 & 1,689 & 1,627 & 1,666 & 1,589 & 1,589 & 1,632 & 1,676 & 1,635 & 1,608 \\
\hline $\begin{array}{l}\text { New nonconcessional external debt contracted or guaranteed by the central government or the BM with maturity } \\
\text { of one year or more (ceiling) }\end{array}$ & 5 & & 5 & 5 & 5 & 5 & 5 & 5 & 5 & 5 & 5 \\
\hline Stock of short-term external public debt outstanding (ceiling) & 0 & & 0 & 0 & 0 & 0 & 0 & 0 & 0 & 0 & 0 \\
\hline External payments arrears (ceiling) & 0 & & 0 & 0 & 0 & 0 & 0 & 0 & 0 & 0 & 0 \\
\hline $\begin{array}{l}\text { Indicative targets: } \\
\text { Balance of the government's savings account set up abroad with proceeds from the coal exploration contract }\end{array}$ & & & & & & & & & & & \\
\hline 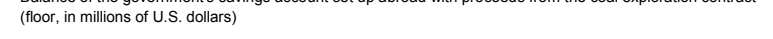 & 0 & & 0 & 0 & 0 & 0 & 0 & 0 & 0 & 0 & 0 \\
\hline Government revenue (floor) & 17,287 & & 17,762 & 26,682 & ... & 37,864 & 37,202 & 9,075 & 20,725 & 32,383 & 44,984 \\
\hline
\end{tabular}

'For definition and adjustors see the attached Program Monitoring Section of Memorandum of Economic and Financial Polices and the Technical Memorandum of Understanding. 
Table 2. Mozambique: Structural Assessment Criteria and Benchmarks Under the Policy Supported Instrument (March-December 2008)

\begin{tabular}{|c|c|c|}
\hline Actions & $\begin{array}{l}\text { Expected Date of } \\
\text { Implementation }\end{array}$ & Comments \\
\hline \multicolumn{3}{|l|}{ Structural assessment criteria } \\
\hline Finalization of a new terms of reference for the Audit Board of the Bank of Mozambique. & June 2008 & Met \\
\hline \multicolumn{3}{|l|}{ Structural benchmarks } \\
\hline $\begin{array}{l}\text { An integrated and e-SISTAFE compatible payroll database will start to be used by the Ministry } \\
\text { of Finance to carry out salary payments via e-SISTAFE. }\end{array}$ & April 2008 & Met \\
\hline A list of additional district and municipalities for the rollout of e-SISTAFE will be defined. & End-June 2008 & Met \\
\hline $\begin{array}{l}\text { The Meticalnet will be adapted to allow for outright operations of treasury bills for the } \\
\text { secondary market. }\end{array}$ & End-June 2008 & Met \\
\hline $\begin{array}{l}\text { The BM adopts an internal audit charter and commences periodic reporting to the Executive } \\
\text { Board on the implementation of audit recommendations. }\end{array}$ & July 2008 & Met \\
\hline $\begin{array}{l}\text { A study, with appropriate technical assistance, will be undertaken that will serve as a basis for } \\
\text { drafting the public enterprises law. }\end{array}$ & December 2008 & $\begin{array}{l}\text { Delayed until } \\
\text { March } 2009\end{array}$ \\
\hline
\end{tabular}


Table 3. Mozambique: Structural Benchmarks Under the Policy Supported Instrument (December 2008-December 2009)

\section{Actions}

Expected Date of Implementation

The Ministry of Finance will approve a PFM vision paper, as the basis for the Sistafe Action Plan and Budget for 2009-2011.

end-March 2009

The Ministry of Industry and Trade will issue a decree streamlining the business inspection process.

end-March 2009

The Government will formally submit a request to start the process to become a member of EITI.

end-June 2009

The Ministry of Finance in coordination with the Ministry of Public Service will prepare an estimate of the new pay policy for the fiscal years 2010-2012.

end-August 2009

A draft public enterprises law will be submitted to the Council of Ministers.

end-September 2009

The central revenue authority and UTRAFE, will implement the pilot project on corporate income tax for the revenue collection module (e-Tributação) of the e-Sistafe.

end-September 2009

The BM with technical assistance support of the Fund will design a plan to strengthen the Monetary Policy framework comprising all intermediate steps, including agreement on a core inflation indicator. 


\section{ATTACHMENT II}

\section{Republic of Mozambique: Technical Memorandum of Understanding on Selected Concepts, Definitions, and Data Reporting Under Mozambique's Policy Support Instrument Program}

October 29, 2008

1. The purpose of this technical memorandum of understanding (TMU) is to describe the concepts and definitions that will be used in monitoring the Policy Support Instrument (PSI) program, including the following:

- $\quad$ net credit to the central government by the banking system;

- $\quad$ government revenue;

- $\quad$ net international reserves, and reserve money of the Bank of Mozambique (BM);

- new nonconcessional external debt contracted or guaranteed by the central government or the BM with a maturity of more than one year;

- $\quad$ short-term external public debt outstanding;

- $\quad$ external payments arrears; and

- $\quad$ foreign program assistance and external debt service.

This memorandum also describes the adjusters that will be applied to certain quantitative assessment criteria of the program.

\section{Definitions}

\section{Net credit to the central government}

2. Net credit to the central government (NCG) by the banking system is defined as the difference between the outstanding amount of bank credits to the central government and the central government's deposits with the banking system, excluding deposits in project accounts with the banking system, recapitalization bonds issued to the BM, and proceeds from the signing fee for coal exploration. Credits comprise bank loans and advances to the government and holdings of government securities and promissory notes. NCG will be calculated based on data from balance sheets of the monetary authority and commercial banks as per the monetary survey. The limits on the change in net credit to the central government by the banking system will be cumulative from end-December of the previous year.

3. The government encompasses all institutions whose revenue and expenditure are included in the state budget (Orçamento do Estado): central government ministries, agencies, 
and the administration of 11 provinces. Although local governments (33 municipalities or autarquias) are not included because they are independent, the bulk of their revenue is registered in the state budget as transfers to local governments.

\section{Government revenue and financing}

4. Revenue is defined to include all receipts of General Directorate of Tax (Direcção Geral dos Impostos or DGI), the General Directorate of Customs (Direcção Geral das Alfândegas, DGA), and nontax revenue, including certain own-generated revenues of districts and some line ministries as defined in the budget. Net receipts from privatization received by the National Directorate of State Assets (Direcção Nacional do Património do Estado) and unrealized profits transferred by the central bank to the treasury will not be considered as revenue (above the line) and will be accounted for as other domestic financing (below the line).

5. For the purpose of program monitoring, revenue is considered as collected at the time when it is received by the DGI from private agents or other government collecting agencies, in cash or checks, or through transfers into a DGI bank account.

\section{Reserve money}

6. Reserve money is defined as the sum of currency issued by BM and the commercial banks' deposits in the BM. The commercial bank deposits include the statutory required reserves and excess reserves held at the BM. The reserve money stock will be monitored by the monetary authority and provided to the IMF by the BM.

\section{Net international reserves}

7. Net international reserves of the BM are defined as reserve assets minus reserve liabilities. The BM's reserve assets include (a) monetary gold; (b) holdings of SDRs; (c) reserve position at the IMF; (d) holdings of foreign exchange; and (e) claims on nonresidents, such as deposits abroad (excluding the government's savings account related to the Moatize coal mine concession). Reserve assets exclude assets pledged or otherwise encumbered, including but not limited to assets used as collateral or guarantee for a thirdparty external liability (assets not readily available.) The BM's reserve liabilities include (a) all short-term foreign exchange liabilities to nonresidents with original maturity of up to and including one year; and (b) all liabilities to the IMF.

8. For the purposes of program monitoring and in order to exclude valuation changes, net international reserves will be measured at the official exchange rates prevailing at end2007 and used by the BM.

9. The BM will publish the exchange rates quoted by commercial banks on average as the market rates. 


\section{New nonconcessional external debt contracted or guaranteed by the central government or the BM with maturity of more than one year}

10. The term 'debt' will have the meaning set forth in Point 9 of the Guidelines on Performance Criteria with Respect to Foreign Debt adopted on August 24, 2000. Government debt is outstanding debt owed or guaranteed by the central government or the BM (but does not include debt of any political subdivision or government-owned entity with a separate legal personality that is not otherwise owed or guaranteed by the central government).

11. The central government will limit contracting or guaranteeing external debt up to US\$5 million each year with original maturity of one year or more with a grant element of less than 35 percent, calculated using currency-specific discount rates based on the Organization for Economic Cooperation and Development (OECD) commercial interest reference rates in accordance with the annual Budget Law. This assessment criterion applies not only to debt as defined in point 9 of the Guidelines on Performance Criteria with Respect to Foreign Debt adopted on August 24, 2000, but also to commitments contracted or guaranteed for which value has not been received. This assessment criterion will be assessed on a continuous basis.

\section{Stock of short-term external public debt outstanding}

12. The central government will not contract or guarantee external debt with original maturity of less than one year. This assessment criterion applies not only to debt as defined in point 9 of the Guidelines on Performance Criteria with Respect to Foreign Debt adopted on August 24, 2000, but also to commitments contracted or guaranteed for which value has not been received. Excluded from this assessment criterion are short-term, import-related trade credits. This assessment criterion will be assessed on a continuous basis.

\section{External payments arrears}

13. The government undertakes not to incur payments arrears on external debt owed or guaranteed by the central government, with the exception of external payments arrears arising from government debt that is being renegotiated with creditors, including Paris Club creditors. This assessment criterion will be assessed on a continuous basis.

\section{Foreign program assistance}

14. Foreign program assistance is defined as grants and loans received by the Ministry of Finance through BM accounts excluding those related to projects (Table 1).

\section{Actual external debt-service payments}

15. Actual external debt-service payments are defined as cash payments on external debtservice obligations of the government and central bank, including obligations to Paris Club 
and other bilateral creditors rescheduled under enhanced HIPC Initiative completion point terms, multilateral creditors, and private creditors, but excluding obligations to the IMF (Table 1).

\section{Adjusters}

\section{Net international reserves}

16. The quantitative targets (floors) for the central bank's net international reserves will not be adjusted for any excess in disbursements of foreign program assistance (and any shortfall in external debt service), compared to the program baseline. These targets will be adjusted downward by 100 percent of any shortfall in external program aid (up to a maximum of US $\$ 100$ million) and any excess in external debt service payments, compared to the program baseline (Table 1).

17. The quantitative targets (floors) for the central bank's net international reserves will be adjusted downward/upward for any revision made to the end-year figures corresponding to the previous year.

18. They will also be adjusted upward for the full amount of any external privatization proceeds in excess of those envisaged in the program, unless these proceeds are deposited in the government's savings account abroad. If they are deposited abroad, the indicative targets on the balance in this account will be adjusted upward for the full amount of the higher than envisaged proceeds, net of any costs related to the privatizations, including severance payments.

19. The quantitative targets (floors) for the central bank's net international reserves will be adjusted downward up to a maximum of US\$50 million per annum by the amount that the actual fuel import bill is higher due to a rise in the average fuel import price. This adjustment will be equal to the difference between the realized and the programmed average fuel import price, multiplied by the volume of total fuel imports progammed for each quarter (Table 1).

20. The quantitative target (floor) on net international reserves will be adjusted downward to accommodate the possible need for higher external outlays to deal with natural disasters, up to a total limit of US\$20 million.

\section{Net credit to government}

21. The quantitative targets (ceilings) for net credit on the central government (NCG) will not be adjusted for any excess in disbursements of foreign program assistance (and any shortfall in external debt service), compared to the program baseline. These targets will be adjusted upward by 100 percent of any shortfall in external program aid (up to a maximum of US\$100 million) and any excess in external debt service payments, compared to the program baseline (Table 1). 
22. These targets will also be adjusted downward for the full amount of any privatization proceeds in excess of those envisaged in the program, unless these proceeds are deposited in the government's savings account abroad.

23. The quantitative targets (ceilings) for NCG will be adjusted downward (upward) for any increase (decrease) in domestic financing from the non-financial private sector. The government expenditures contingent on higher disbursements of foreign program assistance (and lower external debt service) will be used to finance greater "priority" spending identified in the budget.

24. The quantitative target (ceiling) for net credit to the central government (NCG) will be adjusted upward to accommodate the possible need for higher locally financed government outlays to deal with natural disasters, up to a total limit of MT 500 million.

\section{Reserve money}

25. The reserve money ceiling will be adjusted equivalently upward up to a maximum of MT 500 million to the extent that the outstanding stock of currency issued by the BM exceeds those projected in Table 1. The maximum at end-December will be further adjusted by an additional MT 500 million because of the seasonal increase in the demand for currency during the festive season in the fourth quarter of the year.

\section{Data Reporting}

26. In addition to providing the monthly and quarterly data needed to monitor program implementation in relation to the programs' quantitative targets and broader economic developments, the authorities will provide weekly updates of the daily data set out in Table 1 as well as the weekly data set out in Table 4 of the TMU dated May 26, 2005. Monthly updates will also be provided of the foreign exchange cash flow of the BM.

27. The government will continue to provide Fund staff with the data corresponding to monthly government revenues (in detail according to the fiscal table), with a lag not exceeding one month. In addition, the government will continue to publish and provide Fund staff with the quarterly budget execution reports with a time lag not exceeding 45 days.

28. In addition, the government will provide monthly information on the balance of its savings account abroad and will start developing and providing information on domestic arrears on a quarterly basis.

29. From December 2005 onwards, the monetary survey made available by the BM will clearly identify donor-financed project deposits (with a breakdown between foreign and domestic currency) included in net credit to the government in both the central bank's and commercial banks' balance sheets. 
Mozambique: Technical Memorandum of Understanding, 2007-09

\begin{tabular}{|c|c|c|c|c|c|c|c|c|c|c|c|c|c|c|c|}
\hline & \multicolumn{10}{|c|}{2008} & \multicolumn{5}{|c|}{2009} \\
\hline & Q1 & & Q2 & & Q3 & & Q4 & & Year & & Q1 & Q2 & Q3 & Q4 & Year \\
\hline & Country Report 08/220 & Act. & Country Report 08/220 & Act. & Country Report 08/220 & Proj. & Country Report 08/220 & Proj. & Country Report 08/220 & Proj. & Proj. & Proj. & Proj. & Proj. & Proj. \\
\hline Net foreign program assistance (US\$ mn) & 268 & 280 & 77 & 8 & 2 & 76 & 45 & 137 & 392 & 501 & 153 & 105 & 42 & 55 & 356 \\
\hline Gross foreign program assistance & 280 & 292 & 100 & 17 & 14 & 87 & 56 & 156 & 451 & 552 & 164 & 117 & 63 & 69 & 413 \\
\hline Program grants & 218 & 230 & 100 & 17 & 13 & 87 & 17 & 36 & 349 & 371 & 164 & 117 & 63 & 39 & 382 \\
\hline Program loans & 63 & 62 & 0 & 0 & 1 & 0 & 38 & 120 & 102 & 182 & 0 & 0 & 0 & 31 & 31 \\
\hline External debt service & 12 & 12 & 23 & 9 & 12 & 11 & 11 & 20 & 59 & 52 & 11 & 11 & 21 & 14 & 57 \\
\hline Net foreign program assistance (MT mn) & 6,466 & 6,701 & 1,856 & 164 & 38 & 1,747 & 1,106 & 3,337 & 9,465 & 11,950 & 3,776 & 2,338 & 1,203 & 1,258 & 8,575 \\
\hline Gross foreign program assistance & 6,756 & 7,027 & 2,419 & 409 & 335 & 2,085 & 1,359 & 3,808 & 10,870 & 13,330 & 4,055 & 2,905 & 1,574 & 1,771 & 10,305 \\
\hline Program grants & 5,241 & 5,542 & 2,419 & 409 & 320 & 2,085 & 425 & 887 & 8,405 & 8,923 & 4,055 & 2,905 & 1,574 & 990 & 9,523 \\
\hline Program loans & 1,515 & 1,486 & 0 & 0 & 16 & 0 & 934 & 2,921 & 2,465 & 4,407 & 0 & 0 & 0 & 782 & 782 \\
\hline External debt service & 290 & 326 & 563 & 245 & 298 & 338 & 253 & 471 & 1,404 & 1,380 & 279 & 567 & 371 & 513 & 1,730 \\
\hline Stock of outstanding currency (MT bm) & 9,055 & 9,046 & 10,772 & 10,745 & 10,854 & 10,439 & 11,659 & 12,036 & 11,659 & 12,036 & 10,075 & 11,323 & 11,383 & 13,000 & 13,000 \\
\hline Average global oil price, APSP, US\$/barrel & 87 & 95 & 87 & 121 & 86 & 116 & 85 & 96 & 86 & 107 & 72 & 74 & 76 & 78 & 75 \\
\hline Average fuel import price, US $\$ /$ barrel (10 barrels per mt) & 87 & 90 & 84 & 109 & 82 & 84 & 77 & 127 & 83 & 102 & 68 & 67 & 55 & 70 & 65 \\
\hline Volume of fuel imports (1000s mt) & 125 & 141 & 195 & 122 & 183 & 184 & 134 & 173 & 637 & 620 & 154 & 133 & 202 & 280 & 770 \\
\hline Ttoal fuel import bill (USSm) & 109 & 127 & 164 & 133 & 150 & 155 & 103 & 220 & 527 & 635 & 105 & 89 & 110 & 196 & 500 \\
\hline
\end{tabular}


INTERNATIONAL MONETARY FUND

REPUBLIC OF MOZAMBIQUE

\section{Third Review Under the Policy Support Instrument \\ Informational Annex}

Prepared by the African Department

(In consultation with other departments)

December 23, 2008

- Relations with the Fund. Describes financial and technical assistance by the IMF.

- World Bank-IMF Joint Management Action Plan. Constitutes a matrix on work program of both the World Bank and the IMF.

- $\quad$ Statistical Issues. Assesses the quality and timeliness of statistical data and identifies areas for improvement. 


\section{Mozambique: Relations with the Fund}

(as of November 30, 2008)

I. Membership Status: Joined: September 24, 1984;

Article XIV

II. General Resources Account:

SDR Million

113.60

113.60

Fund holdings of currency

Reserve Position

Holdings Exchange Rate

III. SDR Department:

Holdings

IV. Outstanding Purchases and Loans:

PRGF Arrangements
SDR Million

0.09

SDR Million

9.74
$\%$ Quota

100.00

100.00

0.01
\%Allocation

N/A

\%Quota

8.57

V. Latest Financial Arrangements:

$\begin{array}{llcrr}\text { Type } & \begin{array}{c}\text { Date of } \\ \text { Arrangement }\end{array} & \begin{array}{c}\text { Expiration } \\ \text { Date }\end{array} & \begin{array}{c}\text { Amount Approved } \\ \text { (SDR Million) }\end{array} & \begin{array}{c}\text { Amount Drawn } \\ \text { (SDR Million) }\end{array} \\ \text { PRGF } & \text { Jul 06, 2004 } & \text { Jul 05, 2007 } & 11.36 & 11.36 \\ \text { PRGF } & \text { Jun 28, 1999 } & \text { Jun 28, 2003 } & 87.20 & 78.80 \\ \text { PRGF } & \text { Jun 21, 1996 } & \text { Jun 27, 1999 } & 75.60 & 75.60\end{array}$

VI. Projected Payments to Fund ${ }^{1}$

(SDR Million; based on existing use of resources and present holdings of SDRs):

\begin{tabular}{lccccc} 
& \multicolumn{5}{c}{ Forthcoming } \\
\cline { 2 - 6 } & $\underline{2008}$ & $\underline{2009}$ & $\underline{2010}$ & $\underline{2011}$ & $\underline{2012}$ \\
Principal & $\underline{20016}$ & & 0.97 & 1.46 \\
Total & $\underline{0.02}$ & $\underline{0.05}$ & $\underline{0.05}$ & $\underline{0.05}$ & $\underline{0.04}$ \\
\hline
\end{tabular}

1 When a member has overdue financial obligations outstanding for more than three months, the amount of such arrears will be shown in this section. 
VII. Implementation of HIPC Initiative:

\begin{tabular}{|c|c|c|c|}
\hline \multirow[b]{2}{*}{ I. Commitment of HIPC assistance } & \multicolumn{2}{|c|}{ Original Enhanced } & \multirow[b]{2}{*}{$\underline{\text { Total }}$} \\
\hline & Framework & Framework & \\
\hline Decision point date & Apr 1998 & Apr 2000 & \\
\hline Assistance committed & & & \\
\hline by all creditors (US\$ Million) ${ }^{1}$ & $1,716.00$ & 306.00 & \\
\hline Of which: IMF assistance (US\$ million) & 124.60 & 18.46 & \\
\hline (SDR equivalent in millions) & 93.17 & 13.73 & \\
\hline Completion point date & Jun 1999 & Sep 2001 & \\
\hline \multicolumn{4}{|l|}{ II. Disbursement of IMF assistance (SDR Million) } \\
\hline Assistance disbursed to the member & 93.17 & 13.73 & 106.90 \\
\hline Interim assistance & -- & 2.31 & 2.31 \\
\hline Completion point balance & 93.17 & 11.42 & 104.58 \\
\hline Additional disbursement of interest income ${ }^{2}$ & -- & 1.10 & 1.10 \\
\hline Total disbursements & 93.17 & 14.83 & 108.00 \\
\hline
\end{tabular}

${ }_{1}$ Assistance committed under the original framework is expressed in net present value (NPV) terms at the completion point, and assistance committed under the enhanced framework is expressed in NPV terms at the decision point. Hence these two amounts can not be added.

${ }^{2}$ Under the enhanced framework, an additional disbursement is made at the completion point corresponding to interest income earned on the amount committed at the decision point but not disbursed during the interim period.

VIII. Implementation of Multilateral Debt Relief Initiative (MDRI):

\begin{tabular}{|c|c|c|c|c|}
\hline . & MDRI-eligible debt (SDR Million) $^{1}$ & & & 106.56 \\
\hline & Financed by: MDRI Trust & & & 83.05 \\
\hline & Remaining HIPC resources & & & 23.51 \\
\hline \multirow[t]{5}{*}{ II. } & Debt Relief by Facility (SDR Million) & & & \\
\hline & & \multicolumn{2}{|c|}{ Eligible Debt } & \\
\hline & \multicolumn{3}{|l|}{ Delivery } & \\
\hline & Date & GRA & PRGF & $\underline{\text { Total }}$ \\
\hline & January 2006 & N/A & 106.56 & 106.56 \\
\hline
\end{tabular}

1 The MDRI provides 100 percent debt relief to eligible member countries that qualified for the assistance. Grant assistance from the MDRI Trust and HIPC resources provide debt relief to cover the full stock of debt owed to the Fund as of end-2004 that remains outstanding at the time the member qualifies for such debt relief. 


\section{Safeguards assessment}

At the request of the Bank of Mozambique, FIN conducted a voluntary safeguards assessment which was concluded in June 2008. This assessment updated the findings of an earlier assessment completed in August 2004. The 2008 assessment noted progress in implementing the recommendations of the earlier assessment. However, weaknesses remain in the external and internal audit functions, financial reporting, and control systems.

\section{Exchange arrangements}

Mozambique's exchange system is a managed float. Commercial banks may buy and sell foreign exchange to individual customers on a fully negotiable basis. The Bank of Mozambique introduced a foreign exchange auction system in January 2005. Auctions are held bi-weekly.

Mozambique still avails itself of the transitional arrangements under Article XIV of the Fund Articles of Agreement, but has eliminated all Article XIV restrictions. It maintains, however, restrictions on the making of payments and transfers for current international transactions subject to Fund approval under Article VIII, as evidenced by (i) the discretionary prior approval for remittances of family living expenses; (ii) the authorization for the purchase of foreign exchange in excess of US $\$ 5,000$ for certain transactions; (iii) the prohibition for the conversion of balances of nonresidents' domestic currency accounts into foreign currency or transfer abroad; (iv) the prohibition on advance payments for a service; and (v) the prohibition on advance payments for the import of goods. On June 18, 2007, the Fund granted approval for the retention of these five exchange restrictions until May 31, 2008. At the authorities' request, an Article VIII mission was conducted by LEG and PDR in March 2004. A new foreign exchange law was submitted to the Assembly in May 2007. Following enactment of the new law and issuance of related regulations, the authorities intend to accept their obligations under Article VIII sections 2, 3, and 4 of the Fund's Articles of Agreement.

\section{Article IV consultation}

In accordance with Decision No. 12794-(02/76), as amended by Decision No 12854-(02/96), Mozambique is on a 24-month Article IV cycle due to the approval of a PSI in June 2007. The 2007 Article IV consultation was completed by the Executive Board on June 18, 2007 (Country Report No. 07/262).

In concluding the 2007 Article IV consultation, Executive Directors commended the Mozambican authorities on the impressive macroeconomic performance and overall satisfactory program performance since the last Article IV consultation. At the same time, they noted there are policy challenges related to sustaining Mozambique's growth takeoff and to achieving the MDGs. In this regard, they agreed that the strategy to consolidate macroeconomic stability in the context of a continued scaling-up of foreign aid and the 
acceleration of a second wave of reforms outlined in the authorities' poverty reduction plan for 2006-09 (PARPA II) would be essential to overcome such challenges. In particular, they looked forward to the timely implementation of reforms to (i) increasing tax revenues; (ii) strengthening public sector operations; (iii) reducing the costs of doing business; and (iv) improving the management of natural resources.

\section{Ex-post assessment of performance under Fund-supported programs}

An ex post assessment (EPA) of Mozambique's performance under Fund-Supported Programs since 1987 was undertaken in December 2003 under the guidelines on assessments of countries with a longer-term program engagement. The EPA was circulated to the Executive Board on November 21, 2003 (Country Report No. 04/53).

Directors commended the authorities for their pursuit of sound macroeconomic policies and wide ranging structural reforms over the past fifteen years which contributed to strong growth of the economy, and a steady decline in poverty rates. Noting that despite considerable progress Mozambique remains a very poor and vulnerable country, Directors urged the authorities to persevere in their efforts to consolidate macroeconomic stability and accelerate and deepen structural reforms with a view to sustaining economic growth, encouraging employment creation, and further reducing poverty.

\section{FSAP participation and ROSCs}

A Financial Sector Assessment Program (FSAP) for Mozambique was undertaken during the first quarter of 2003. The related Financial Sector Stability Assessment was circulated to the Executive Board on November 19, 2003 (Country Report No. 04/52). An update to the FSAP is scheduled in early 2009. A ROSC on fiscal transparency was issued on February 22, 2001. This ROSC was updated in the context of the 2002 Article IV consultation (Country Report No. 02/140) and the 2003 Article IV consultation (Country Report No. 04/50). The ROSC on fiscal transparency was updated in May 2008. A Report on the Observance of Standards and Codes (ROSC) data module was prepared in June 2002 and issued on March 5, 2003. This data module was updated in August 2005.

\section{Management's visit}

At the invitation of the authorities, Mr. Kato, Deputy Managing Director, visited Maputo, Mozambique in July 2005.The Managing Director visited Mozambique on August 2007 for a meeting with the African Consultative Group on Quotas, Voice, and Representation. 


\begin{tabular}{|c|c|c|c|c|}
\hline \multicolumn{5}{|c|}{$\begin{array}{l}\text { IMF Technical Assistance Provided to Mozambique } \\
\qquad(2005-08)\end{array}$} \\
\hline Departments & Timing & Form & Purpose & Counterparts \\
\hline Finance & February 2008 & Mission & Update of safeguard assessment & Bank of Mozambique \\
\hline \multirow[t]{13}{*}{ Fiscal Affairs } & April-May 2008 & Mission & Revenue Administration reform & Ministry of Finance \\
\hline & Oct-Nov 2007 & Mission & Tax Administration inspection & Ministry of Finance \\
\hline & September 2007 & Mission & $\begin{array}{l}\text { Public Private Partnerships and } \\
\text { Fiscal Risks }\end{array}$ & Ministry of Finance \\
\hline & August 2007 & Peripatetic expert & Tax Policy and Administration & Ministry of Finance \\
\hline & August 2007 & Mission & $\begin{array}{l}\text { Fiscal ROSC update and Public } \\
\text { Financial Management inspection }\end{array}$ & Ministry of Finance \\
\hline & April-May 2007 & Mission & Tax Administration inspection & Ministry of Finance \\
\hline & April-May 2007 & Mission & Petroleum Fiscal Regime & $\begin{array}{l}\text { Ministry of Finance } \\
\text { Ministry of Mineral } \\
\text { Resources }\end{array}$ \\
\hline & December 2006 & Mission & Tax Administration & Ministry of Finance \\
\hline & March 2006 & Mission & Tax Policy review & Ministry of Finance \\
\hline & November 2005 & Mission & Public expenditure management & Ministry of Finance \\
\hline & October 2005 & $\begin{array}{l}\text { Fourth multipartite } \\
\text { review of the joint } \\
\text { IMF/SECO/ DANIDA } \\
\text { tax and customs } \\
\text { administration reform } \\
\text { project; inspection } \\
\text { mission }\end{array}$ & $\begin{array}{l}\text { Reform of the tax system and its } \\
\text { administration }\end{array}$ & Ministry of Finance \\
\hline & May 2005 & Inspection mission & Public expenditure management & Ministry of Finance \\
\hline & April-May 2005 & $\begin{array}{l}\text { Mid-term review of the } \\
\text { joint } \\
\text { IMF/SECO/DANIDA } \\
\text { domestic tax and } \\
\text { administration reform } \\
\text { project }\end{array}$ & $\begin{array}{l}\text { Reform of the tax system and its } \\
\text { administration; advice on the } \\
\text { creation of a Central Revenue } \\
\text { Authority. }\end{array}$ & Ministry of Finance \\
\hline \multirow[t]{4}{*}{$\begin{array}{l}\text { Monetary and } \\
\text { Capital Markets }\end{array}$} & May 2008 & Mission & $\begin{array}{l}\text { Strengthening Monetary Policy } \\
\text { Formulation and Implementation }\end{array}$ & Bank of Mozambique \\
\hline & December 2007 & Mission & $\begin{array}{l}\text { Implementation of IFRS for Central } \\
\text { Bank accounts. }\end{array}$ & Bank of Mozambique \\
\hline & August 2007 & Mission & $\begin{array}{l}\text { Post-FSAP TA assessment } \\
\text { banking supervision, monetary } \\
\text { Policy and operations }\end{array}$ & Bank of Mozambique \\
\hline & April 2007 & Short-term consultant & Banking supervision & Bank of Mozambique \\
\hline
\end{tabular}




\begin{tabular}{|c|c|c|c|c|}
\hline \multicolumn{5}{|c|}{$\begin{array}{l}\text { IMF Technical Assistance Provided to Mozambique } \\
\qquad(2005-08)\end{array}$} \\
\hline \multirow[t]{7}{*}{ Departments } & Timing & Form & Purpose & Counterparts \\
\hline & October 2006 & Mission & $\begin{array}{l}\text { Post- FSAP TA assessment } \\
\text { banking supervision, monetary } \\
\text { Policy and operations }\end{array}$ & Bank of Mozambique \\
\hline & Feb-March 2006 & Mission & AML/CFT legislation & Bank of Mozambique \\
\hline & Jan-Feb 2006 & Short-term consultant & Monetary policy framework & Bank of Mozambique \\
\hline & Jan-Feb 2006 & Short-term consultant & Monetary operations & Bank of Mozambique \\
\hline & January 2006 & Short-term consultant & Exchange rate management & Bank of Mozambique \\
\hline & September 2005 & Mission & $\begin{array}{l}\text { Post- FSAP TA assessment } \\
\text { banking supervision, monetary } \\
\text { Policy and operations }\end{array}$ & Bank of Mozambique \\
\hline \multirow[t]{9}{*}{ Statistics } & $\begin{array}{l}\text { March-April } \\
2008\end{array}$ & Mission & National accounts statistics & $\begin{array}{l}\text { National Institute of } \\
\text { Statistics }\end{array}$ \\
\hline & February 2008 & Mission & Balance of payments statistics & Bank of Mozambique \\
\hline & December 2007 & Mission & Balance of payments statistics & Bank of Mozambique \\
\hline & November 2007 & Mission & Monetary and financial statistics & Bank of Mozambique \\
\hline & June-July 2006 & Mission & Consumer price statistics & $\begin{array}{l}\text { National Institute of } \\
\text { Statistics (INE) }\end{array}$ \\
\hline & $\begin{array}{l}\text { August 2005- } \\
\text { July } 2006\end{array}$ & Long-term consultant & National accounts statistics & $\begin{array}{l}\text { National Institute of } \\
\text { Statistics }\end{array}$ \\
\hline & July 2005 & Mission & Government finance statistics & $\begin{array}{l}\text { National Institute of } \\
\text { Statistics }\end{array}$ \\
\hline & June-July 2005 & Mission & Consumer price statistics & $\begin{array}{l}\text { National Institute of } \\
\text { Statistics }\end{array}$ \\
\hline & May 2005 & Mission & Balance of payments statistics & Bank of Mozambique \\
\hline
\end{tabular}

Resident Representative: Mr. Felix Fischer has been the IMF's resident representative to Mozambique since March 14, 2006. 


\begin{tabular}{|c|c|c|c|}
\hline \multicolumn{4}{|c|}{ Mozambique: World Bank-IMF Joint Management Action Plan } \\
\hline Title & Products & Timing of Missions & Expected delivery date \\
\hline \multicolumn{4}{|c|}{$\begin{array}{l}\text { A. Mutual information on relevant work } \\
\text { program in next } 12 \text { months }\end{array}$} \\
\hline \multirow[t]{4}{*}{ Bank work program } & CEM on export competitiveness and regional integration & Feb-May 2008 & Jan-2009 \\
\hline & $\begin{array}{l}\text { PRSC5 supporting government reforms program in PFM, governance and } \\
\text { economic development reforms }\end{array}$ & & Nov 2008 (delivered) \\
\hline & FSTAP project support to financial sector reforms & Oct-08 & ongoing \\
\hline & ESW on inter-goverrnmental fiscal architecture (for decentralization) & & July 2008 (delivered) \\
\hline \multirow[t]{4}{*}{ Fund work program } & Third Review of the PSI & Oct-08 & Dec-08 \\
\hline & Follow up mission on Tax Administration & Apr-08 & May-08 \\
\hline & Inspection mission on PFM & Aug- 08 & Sep-08 \\
\hline & $\begin{array}{l}\text { Follow up mission on Monetary Policy and Operations, Banking } \\
\text { Supervision and Accounting }\end{array}$ & Sep-08 & Nov-08 \\
\hline \multicolumn{4}{|c|}{$\begin{array}{l}\text { B. Requests for work program inputs } \\
\text { with summary justification }\end{array}$} \\
\hline \multirow[t]{2}{*}{ Bank request to the Fund } & Updated Macro Framework data and PSI review Staff Report & PSI reviews & April and October 2009 \\
\hline & $\begin{array}{l}\text { Board Chairman Summing up from PSI review discussion (or Assessment } \\
\text { letter) not older than six-months }\end{array}$ & & Sep-09 \\
\hline Fund request to the Bank & $\begin{array}{l}\text { Updates on advancing structural reforms: procurement, decentralization, } \\
\text { business environment, financial sector }\end{array}$ & & March and September 2008 \\
\hline \multicolumn{4}{|c|}{$\begin{array}{l}\text { C. Agreements on joint products and } \\
\text { missions }\end{array}$} \\
\hline \multirow[t]{8}{*}{ Joint products } & Policy advice on subsidies (food and energy) & May-September 2008 & June 2008 (delivered) \\
\hline & $\begin{array}{l}\text { Policy discussion on improving the domestic benefits of large investment } \\
\text { projects }\end{array}$ & Feb-May 2008 & May 2008 (delivered) \\
\hline & Policy advice on Public Sector Salary Reforms (incl. pensions) & ongoing & ongoing \\
\hline & FSAP & Feb-09 & Apr-09 \\
\hline & $\begin{array}{l}\text { PFM update work plan for 2009-11 in light of findings from PEFA and } \\
\text { ROSC }\end{array}$ & Meeting in February 2009 & Apr-09 \\
\hline & PFM: continue to monitor consolidation and expansion of e-SISTAFE. & ongoing & ongoing \\
\hline & DSA & Oct-08 & Dec-08 \\
\hline & Support to EITI membership, and scoping report on EITI++ & ongoing & Oct 2008 (delivered) \\
\hline
\end{tabular}




\section{Mozambique: Statistical Issues}

1. Data provision has some shortcomings, but is broadly adequate for surveillance. Despite the increase in budget resources allocated to the compilation of official statistics, continued high reliance on external funding raises concerns about the sustainability of the programs of the National Institute of Statistics (INE). The authorities are making efforts to improve the quality and timeliness of economic and financial data through participation in the Fund's General Data Dissemination System (GDDS) and in the external sector module of the GDDS Project for Anglophone African Countries (funded by the U.K. Department for International Development (DFID)). GDDS metadata have been posted on the Fund's Dissemination Standards Bulletin Board (DSBB) since November 2003.

2. In May 2005, a STA mission updated the data module of the Report on the Observance of Standards and Codes (ROSC) prepared in June 2002. The mission noted that significant effort has been made to address previously identified shortcomings. The improvements in the institutional environment and the increased allocation of resources for the compilation of national accounts, balance of payments, and more recently, government finance statistics should help to address weaknesses in the prerequisites for the quality of the statistics. The methodological soundness, accuracy, and reliability of macroeconomic statistics are starting to show improvements as a result of these actions.

\section{National accounts}

3. The national accounts are prepared by the INE in accordance with the 1993 System of National Accounts (1993 SNA). In August 2007, the INE released for the first time quarterly GDP estimates at constant prices covering 2000-07, along with a revised annual GDP series for the period 1991-2006, having 2003 as the new base year. A (March-April 2008) STA mission advised the authorities on further improvements needed to advance the quarterly national accounts compilation at current prices, that could be released starting in mid-2009. The INE has launched a new household income and expenditure survey and new economic censuses leading to a new business registry. Furthermore, it is compiling more comprehensive and timely foreign trade data based on improved classification systems.

\section{Prices and labor market}

4. A revamped consumer price index for Maputo based on weights derived from the 2002-03 household survey was released in February 2006. A national index obtained by integrating the indices for Maputo, Beira, and Nampula was released in April 2006. A STA mission in June 2006 found important advances regarding the use of the COICOP classification and the strengthening of human resources in the compilation of the CPI. Several issues remain outstanding, however, such as price collection procedures not fully consolidated at the provincial level, imputation methods, and possible inconsistencies in the overlapping period between the new and old series. 
5. There are insufficient sectoral labor market and employment data, and where available, they have limited coverage. A one-year labor market survey of the entire country, undertaken by INE in collaboration with the Labor Ministry, was launched in October 2004.

\section{Monetary statistics}

6. A STA mission in September 2004 prepared a work plan for the implementation of the Monetary and Financial Statistics Manual (MFSM) and the development of an integrated monetary database (IMD) to meet the needs of the Bank of Mozambique (BM), AFR, and STA. The mission recommended improving the classification and valuation of some financial instruments, estimating the full instrument and sectoral breakdown based on available information, and expanding the coverage of the survey on other depository corporations to include credit cooperatives.

7. A follow-up monetary and financial statistics mission in November 2007 assisted the authorities in finalizing the IMD, which allow the derivation of accurate and timely monthly monetary statistics, while reducing BM's reporting burden. Mozambique now reports monthly monetary data to STA using the standardized reporting forms (SRFs). As a result, five-year historical enhanced data are being published in the International Financial Statistics (IFS) Supplement.

\section{External sector statistics}

8. With assistance from STA, provided in the context of the GDDS regional project, the $\mathrm{BM}$ has made significant progress toward compiling and disseminating balance of payments (BOP) and international investment position (IIP) statistics that are fully aligned with the Balance of Payments Manual, fifth edition (BPM5).

9. The BM has an adequate institutional framework for the compilation of BOP and IIP statistics, and has implemented many of the recommendations made by the four technical assistance missions conducted since mid-2003. However, in order for the work on institutional capacity building to be consolidated, the Foreign Exchange Law has to be approved (incorporating the definition of residency in line with the BPM5 methodology), training on BOP statistics has to be sustained, and the project to computerize the balance of payments compilation system has to be completed. Although, major improvements were achieved in the basic data sources since the beginning of the project, remaining concerns include (i) the coverage and quality of the data obtained in the enterprise surveys; (ii) the quality of external trade data, especially with regard to price and volume indices; (iii) the coverage, time of recording, and classification of the data on foreign investment and private loans; (iv) completeness of data for the International Reserves Template and their reconciliation with the reserve component of the balance of payments; and (v) compiling the IIP using the sources that are used for the balance of payments compilation. To improve the collection, timeliness, and quality of merchandise trade data, a panel on foreign trade 
statistics was established, comprising representatives from the Directorate General of Customs, National Statistics Institute, and BM.

10. In 2007, the BM started to compile and report to the STA comprehensive IIP data. Previously, only partial IIP data had been reported and published in the Balance of Payments Statistics Yearbook.

\section{Government finance statistics}

11. A July 2005 STA mission found serious weaknesses in fiscal statistics. The budget accounting system, broadly aligned to the GFSM 1986 analytical framework, had shortcomings relating to: definitions and coverage of institutional units included in the general government sector; budgetary classifications not fully meeting GFS needs; and the omission of certain revenues and expenditures. Mozambique does not report fiscal data for publication in the IFS or the Government Finance Statistic Yearbook (GFSY). 
Mozambique: Table of Common Indicators Required for Surveillance

(As of December 4, 2008)

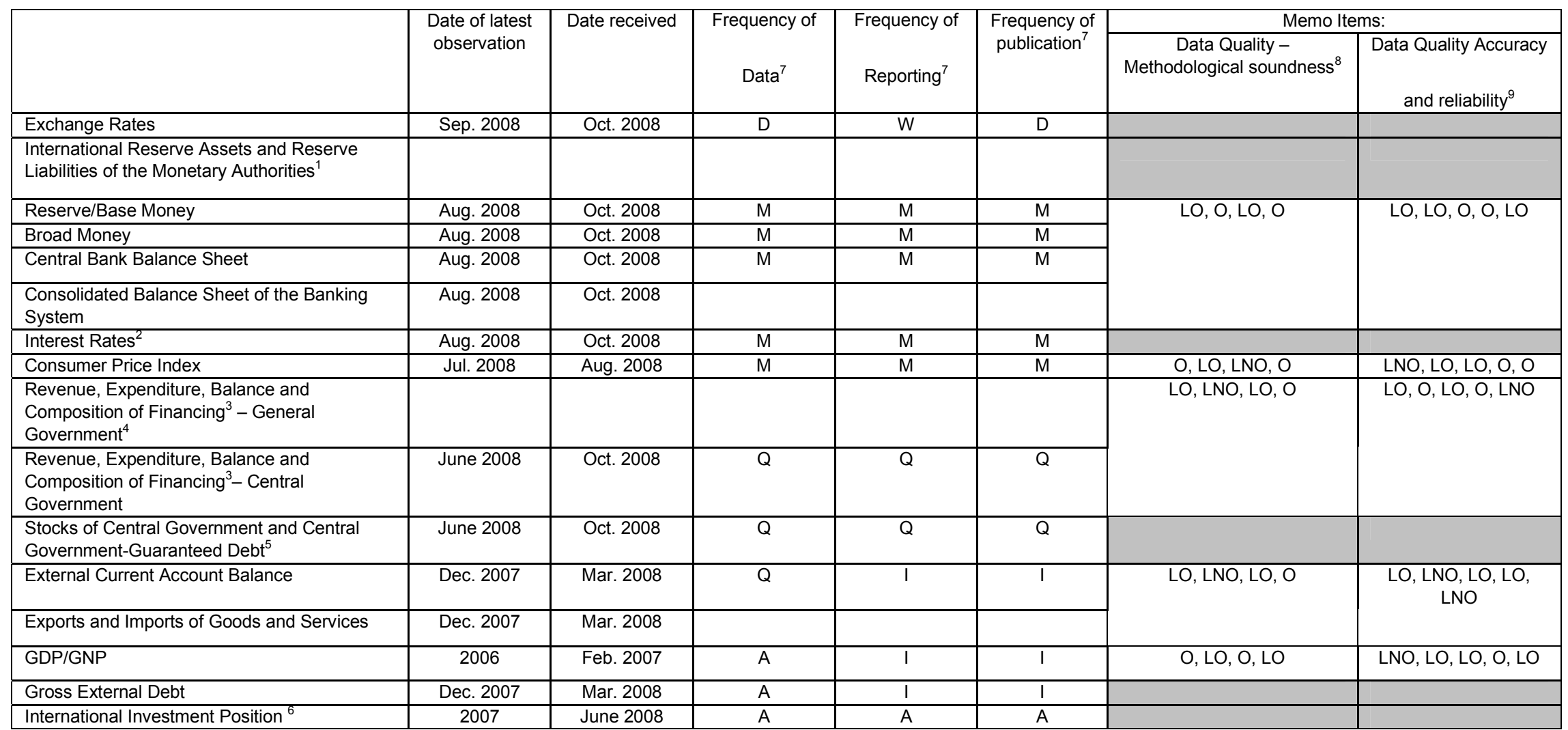

${ }^{1}$ Includes reserve assets pledged or otherwise encumbered as well as net derivative positions.

${ }^{2}$ Both market-based and officially determined, including discount rates, money market rates, rates on treasury bills, notes and bonds.

${ }^{3}$ Foreign, domestic bank, and domestic nonbank financing.

${ }^{4}$ The general government consists of the central government (budgetary funds, extrabudgetary funds, and social security funds) and state and local governments.

Including currency and maturity composition.

${ }^{6}$ Includes external gross financial asset and liability positions vis-à-vis nonresidents.

${ }^{7}$ Includes external gross financial asset and liability positions vis-à-vis nonresidents.

${ }^{8}$ Daily (D), Weekly (W), Monthly (M), Quarterly (Q), Annually (A), and Not Available (NA).

${ }^{9}$ Reflects the assessment provided in the data ROSC or the Substantive Update for the dataset corresponding to the variable in each row. The assessment indicates whether international standards concerning

concepts and definitions, scope, classification/sectorization, and basis for recording are fully observed (O); largely observed (LO); largely not observed (LNO); not observed (NO); and not available (NA). 


\title{
INTERNATIONAL DEVELOPMENT ASSOCIATION AND INTERNATIONAL MONETARY FUND
}

\author{
REPUBLIC OF MOZAMBIQUE
}

\section{Joint World Bank/IMF Debt Sustainability Analysis}

\author{
Prepared by the staffs of the International Development Association and \\ the International Monetary Fund \\ Approved by Carlos Braga and Sudhir Shetty (World Bank) \\ and Mark Plant and Dominique Desruelle (IMF)
}

December 23, 2008

The joint IMF-World Bank low-income country debt sustainability analysis (LIC DSA) indicates that Mozambique's risk of debt distress remains classified as low. ' The country's external debt is expected to remain well below the indicative thresholds both under the baseline and under various stress tests. Similarly, public debt is expected to decline over the medium-to-long term under the baseline. Stress tests indicate, however, that the public debt trajectory could become unsustainable if the country continues running primary deficits at the order of 4 percent of GDP as expected in 2008. With substantially lower real growth rates over the projection period, public debt could also rise to an unsustainable level over time.

\section{BACKGROUND}

1. At end-2007, Mozambique's external public and publicly guaranteed (PPG) debt stock, including arrears, ${ }^{2}$ was US\$3.3 billion in nominal terms or US\$832 million in NPV terms (Table 1). Of this, 47 percent was owed to multilateral creditors, 53 percent to bilateral creditors, and less than 1 percent to commercial creditors. Thanks to the Multilateral Debt Relief Initiative (MDRI), from the African Development Fund (AfDF), the

\footnotetext{
${ }^{1}$ The 2006 and 2007 DSAs already concluded that the risk of debt distress for Mozambique was low, even with the inclusion of the domestic debt, which was absent in the 2006 DSA. Some of the expected bilateral debt relief agreements envisaged in the 2007 DSA did not materialize but are expected to be concluded during the course of this year.

${ }^{2}$ As of end-2007, arrears to bilateral creditors amounted to approximately US\$959 million of which US\$799 million to Paris Club creditors and US\$160 million to non-Paris Club creditors. This amount was reduced substantially through a debt relief agreement concluded with Portugal, and will further be reduced when Japan and Russia deliver their debt relief. Following the a debt-buy back in 2007, there were no more arrears to commercial creditors.
} 
International Development Association (IDA) of the World Bank, and the IMF, Mozambique's debt stock fell by US $\$ 1.9$ billion in nominal terms in $2006 .{ }^{3}$

Table 1. Mozambique: External and Domestic Nominal Debt Outstanding at End-2007

\begin{tabular}{|c|c|c|c|}
\hline & $\begin{array}{c}\text { Millions } \\
\text { of U.S. dollars }\end{array}$ & $\begin{array}{c}\text { Percent } \\
\text { of total external debt }\end{array}$ & Percent of GDP \\
\hline Public and Publicly Guaranteed Debt & $3,660.2$ & 71.8 & 42.1 \\
\hline Domestic Debt ${ }^{1}$ & 337.6 & 6.6 & 3.9 \\
\hline External Debt & $3,322.6$ & 65.2 & 38.2 \\
\hline Multilateral Creditors & $1,549.1$ & 30.4 & $\mathbf{1 7 . 8}$ \\
\hline IDA & 902.1 & 17.7 & 10.4 \\
\hline IMF & 15.3 & 0.3 & 0.2 \\
\hline African Development Bank & 295.0 & 5.8 & 3.4 \\
\hline Other Multilaterals & 336.7 & 6.6 & 3.9 \\
\hline Bilateral Creditors $^{2}$ & $1,763.6$ & 34.6 & 20.3 \\
\hline Paris Club & 799.5 & 15.7 & 9.2 \\
\hline Non-Paris Club & 964.1 & 18.9 & 11.1 \\
\hline Commercial Creditors $^{2}$ & 9.9 & 0.2 & 0.1 \\
\hline Total Private Non-guaranteed Debt & $1,436.0$ & 28.2 & 16.5 \\
\hline \multicolumn{4}{|l|}{ Memorandum items: } \\
\hline Total External debt & $5,096.1$ & 100.0 & 58.6 \\
\hline PV of external debt & $2,275.4$ & - & 28.2 \\
\hline PV of PPG external debt & 839.4 & - & 10.4 \\
\hline
\end{tabular}

\section{Mozambique had previously benefited from assistance under the HIPC Initiative} provided by multilateral and Paris Club bilateral creditors. ${ }^{4}$ At end-2007, bilateral agreements were reached with all Paris Club creditors except Portugal, Russia, and Japan, who together accounted for about 20 percent of total Paris Club debt in NPV terms at end2000, the reference year for enhanced HIPC debt relief. In July 2008, an agreement with Portugal was signed, in which Portugal canceled all Mozambican debt which amounted to US\$393.5 million. The Mozambican authorities are making best efforts to reach agreement

\footnotetext{
${ }^{3}$ The amount of MDRI relief provided by the AfDF was US $\$ 464.5$ million; IDA provided US $\$ 1.3$ billion; and IMF provided US\$120.6 million.

${ }^{4}$ See "Mozambique-HIPC Debt Initiative: President's Memorandum and Recommendation and Completion Point Document" (IDA/R99-139), and "Mozambique-Enhanced HIPC Debt Initiative: President's Memorandum and Recommendation and Completion Point Document" (IDA/R2001-0150).
} 
on debt cancellation with Japan and Russia, both of which have announced their intentions to cancel a 100 percent to HIPCs that reach completion point.

3. Mozambique has experienced some difficulties in negotiating debt relief agreements with some of its non-Paris Club bilateral creditors. Negotiations are still ongoing with Algeria, Angola, Bulgaria, India, Iraq, Libya, and Poland. Negotiations with Libya are reportedly difficult. The Chinese government cancelled Mozambique's outstanding debt in February 2007, Hungary cancelled 96.75 percent of Mozambique's debt in September 2007, and Kuwait provided comparable treatment with the Paris Club.

4. Mozambique benefited from a commercial debt buy-back operation in 2007. This operation covered all outstanding commercial debt in arrears, which amounted to US\$153 millions. ${ }^{5}$ The Government of Norway and IDA financed the operation through grants.

5. Total external debt in Mozambique has a significant private component due to "megaproject" related lending. Most of the megaproject investments were debt-creating. In 2007, mega projects borrowing were mainly explained by Cahora Bassa's large borrowing of about US $\$ 805$ million. Private external debt accounts for 28 percent of the country's total external debt at end-2007 (Table 1).

6. Mozambique's domestic government debt stock is dominated by recapitalization bonds. The total stock of domestic government debt was about 3.9 percent of GDP at end2007, which is low by regional levels. The Ministry of Finance is committed to non-recourse to domestic financing and has not issued treasury bills for deficit financing purposes since 2006. The current stock of outstanding treasury bills has all been issued by the Bank of Mozambique for sterilization purposes. The majority of the stock of treasury bonds is accounted for by bonds issued to strengthen the central bank's balance sheet in 2005, 2006 and 2007, and related to the process of restructuring commercial banks, which is now complete.

7. A debt management reform program is ongoing, which should address some of the weaknesses in debt management. Debt management functions have recently been moved from the Central Bank to the Treasury, and a process is underway to organize operations according to international standards. The government is also working on a debt strategy, which is expected to be published in 2009 following consultations with stakeholders. The debt office has a complete set of external debt records, but the way disbursements are processed at present often leads to delays in accurately recording the amounts outstanding and disbursed. On the domestic debt side, there is no complete set of data originating from honored guarantees. The debt office does not currently produce a statistical bulletin, although its annual report of activities does contain some debt data.

\footnotetext{
${ }^{5}$ The amount was initially estimated at US\$175.4 million at end-2006.
} 


\section{Methodology AND Key Assumptions}

8. Following the guidelines of the LIC debt sustainability framework, staffs have analyzed the evolution of the external public debt stock and debt service indicators for Mozambique under a baseline scenario and a series of stress tests. ${ }^{6}$ The stress tests are designed to assess the probability of Mozambique facing debt distress in the future under a set of shocks.

9. The analysis is guided by indicative, performance-based debt burden thresholds, which take into account the empirical finding that the debt levels that a low-income country can sustain increase with the quality of its policies and institutions. The quality of policies and institutions is measured by the three-year average of Country Policy and Institutional Assessment (CPIA) scores of the World Bank, according to which Mozambique ranks as a 'medium performer'. The indicative external debt burden thresholds for countries in this category are an NPV of debt-to-exports ratio of 150 percent, an NPV of debt-torevenue ratio of 250 percent, an NPV of debt-to-GDP ratio of 40 percent, and debt-serviceto-exports and debt-service-to-revenue ratios of 20 and 30 percent, respectively.

10. The baseline scenario is subject to a number of assumptions. The underlying macroeconomic assumptions, summarized in Box 1, are consistent with the medium-term macroeconomic framework described in the IMF staff report for the $3^{\text {rd }}$ review under the PSI. In addition, the external debt numbers take into account the debt cancellation signed with Portugal in July 2008 and assume the full delivery of HIPC debt relief by all creditors, 100 percent debt reduction by Russia and Japan. Finally, the authorities are assumed to borrow predominantly from the IDA and AfDF, resulting in an average grant element on new borrowing of 50 percent over the projection period. This assumption is based on the authorities' medium-term projections, which were informed by consultations with donors.

\section{EXTERnAL DEbT SuSTAInABILITY}

11. Under the baseline scenario presented in Table 2, all the debt indicators remain below their respective thresholds. The PV of PPG external debt-to-GDP ratio is projected to rise from 11.6 percent in 2008 to 20.8 percent by 2021, after which it slowly declines over the remainder of the projection period to 19.1 percent by 2028. It thus remains well below the country-specific threshold of 40 percent. The PV of PPG debt-to-exports ratio increases from 35.7 percent in 2008 to 72.5 percent by 2017 - also far below the threshold of 150 percentbefore falling back to about 49.4 percent again by 2028. The NPV of PPG debt-to-revenue ratio increases to a peak of 107.1 percent in 2017, significantly below the threshold of

\footnotetext{
${ }^{6}$ The executive Boards of the Fund and the Bank approved the Operational Framework for Debt Sustainability Assessments in Low-Income Countries in April 2005 and reviewed it in April 2006 and in November 2006 (www.imf.org).
} 
250 percent. It then declines rapidly to 69.9 percent by 2028 , driven in part by the assumed increase in revenue collection.

12. The debt service indicators also remain below their thresholds under the

baseline. As a result of the assumed full delivery of remaining HIPC debt relief in 2008, the PPG debt would fall from 2.6 percent in 2006 to 1.2 percent in 2008 . It would then increase to 4.0 percent in 2023 , then decreasing to 3.6 percent by 2028 , and always well below the 20 percent threshold. The ratio of PPG debt service to fiscal revenues falls from 3.9 percent in 2007 to 2.2 percent in 2009, increasing slowly to a peak of 5.8 percent in 2023 before declining to 5.1 by 2028 , well below the 30 -percent threshold (Table 2).

\section{As in the 2007 DSA, external debt sustainability appears resilient to a number of} shocks. As indicated in Table 3 and Figure 1, even under the most extreme shocks, all the ratios would remain below their indicative threshold. The debt ratios appear particularly sensitive to a 30 percent nominal depreciation against the dollar (scenario B6) and to a decline in transfers and FDI (scenario B4). In scenario B6, the ratio of NPV of debt-to GDP would reach 29 percent in 2018, the NPV of debt-to-revenue ratio would reach 150 percent. In both cases this is a 40 percent increase over the baseline. In scenario B4, the NPV of debtto GDP would jump from 12 percent in 2008 to 24 percent in 2010 and 28 percent in 2018; the debt-to-export ratio would almost double by 2009 to 70 percent and 100 percent as early as 2013; and the debt-to-revenue would reach 143 percent in 2018. Debt ratios would, under all scenarios, remain very low, but appear particularly sensitive to the depreciation of the currency and to borrowing on terms less favorable than in the baseline.

\section{Public Sector debt Sustainability}

\section{Mozambique's public debt (including domestic debt) as percent of GDP is} expected to increase steadily until 2016 and then begin to decline under the baseline scenario (Table 4 and Figure 2). These projections rest on the assumption that there will be no recourse to domestic financing over the long run. In addition, the revenue to GDP ratio will continue to increase (by 0.5 percent per year until 2011 and then by a smaller magnitude afterward) and foreign aid gradually falls as a percentage of GDP (Box 1), while government expenditures will increase only gradually over time. After peaking at about 35 percent of GDP, the debt ratio eventually falls back to close to its 2008 level of about 25 percent of GDP. This debt dynamics is largely driven by developments in external debt, given the low and declining level of domestic debt as a result of the discontinuation of treasury bill issuance for deficit financing purposes, the gradual redemption of bonds issued to restructure the banking system, and predominant use of foreign exchange sales for sterilization purposes. The NPV of public sector debt-to GDP ratio shows a similar pattern over time, as do the NPV of debt-to-revenue and debt service-to-revenue ratios. However, the NPV of debt-torevenue ratio falls faster and to a significantly lower level than in 2008 after reaching its peak in 2017, whereas the debt service-to-revenue ratio begins to fall much later (in 2024) and more slowly. 
15. The large proportion of external debt makes the public debt burden vulnerable to the same set of shocks as external debt. There are, however, some additional risks related to the potential sterilization needs to mop up excess liquidity, which would increase in the event of lower revenues and/or higher expenditures - even if financed by higher aid, as long as its disbursement is volatility and/or there is reluctance to sell foreign exchange due to fears of possible Dutch-Disease effects of aid inflows. The stress tests indicate that public sector debt ratios are most vulnerable to a persistent large primary deficit and a permanent lower GDP growth, which would result in sharp increases in the public debt ratios over time.

\section{Conclusions}

16. In staffs' view, Mozambique faces a low risk of debt distress. Mozambique's external debt levels are expected to remain well below their indicative thresholds for debt distress both under the baseline and under various stress tests. Mozambique's public debt is expected to decline over the medium-to-long term under the baseline, while the stress tests indicate that public sector debt ratios are most vulnerable to a permanently large primary deficit and lower GDP growth.

17. A prudent new borrowing policy and fiscal stance remain important. Where possible, the authorities should continue to rely on concessional borrowing and grants to minimize future debt service, and any non-concessional financing of new projects ought to be considered case by case based on economic return, impact on debt sustainability, and potential effects on the financing decisions of donors and concessional lenders. Also, the authorities would still need to closely monitor any substantial scaling-up of concessional external borrowing and to avoid issuance of a large amount of domestic debt to sterilize the additional liquidity injected from increased government spending. 


\section{Box 1. Macroeconomic Assumptions 2007-28}

The medium-term assumptions in the baseline scenario for 2008-28 are consistent with the medium-term macroeconomic framework described in the IMF staff report for the $3^{\text {rd }}$ review under the PSI and in the authorities' medium-term macroeconomic framework described in the government's Plano de Acção para Redução da Pobreza Absoluta II (PARPA II).

Real GDP growth is projected to average 6.5 percent per year during 2008-12 and 6.8 percent thereafter, representing a deceleration from the annual average of 8.4 percent achieved during 2001-07.

Consumer price inflation is projected to fall to about $4 \frac{1}{2}$ percent during $2008-28$, as oil and food prices stabilize.

Export growth is projected to accelerate slowly from about 5 percent per year over 2008-13 to about 8 percent thereafter. This is driven to a large extent by the growth of traditional (non-megaproject) exports at the rate of import demand growth in Mozambique's trade partners and by the prospects for megaproject exports that are affected by world prices of aluminum and gas.

Import growth is projected to increase slightly from about 6 percent per year over the period 2008-13 to 7 percent in the long term. Import growth associated with megaprojects is assumed to equal the rate of megaproject export growth, assuming that the import content of megaprojects remains roughly constant on average. All other imports are assumed to grow at the rate of real GDP growth.

The non-interest current account deficit after grants is projected to decline slightly from 5.5 percent of GDP during 2008-13 to 5.0 percent in 2013-26.

Fiscal revenue is expected to rise from about 15 percent of GDP in 2006 to just under 18 percent of GDP in 2011, largely reflecting a 0.5 percent of GDP annual revenue effort on account of improved revenue administration and a broadening tax base. ${ }^{7}$ Over time, non-tax revenues from natural resource exploitation, particularly megaprojects, are expected to make a growing fiscal contribution, but the increase of overall revenue effort slows somewhat after 2011. Nevertheless, total revenue is projected to reach about $23 \frac{1}{2}$ percent of GDP by 2028, of which about 21 percent of GDP comes from taxes, a level close to Mozambique's potential tax ratio as estimated by a number of studies. ${ }^{8}$ Total expenditures as a percent of GDP are projected to increase only moderately over time.

External financing. External grants are projected to remain high over the medium term. They would nonetheless decline slightly from an average of 13.5 percent of GDP during 2001-07 to 10.8 percent during 2008-15. No grant financing is assumed to come forward from IDA after 2008 (a grant of US $\$ 10$ million was provided in 2008 from IDA's Food Price Crisis Response Trust Fund). Public sector loans for the period 2008-15 are projected to remain at their average level during 2005-07, or 4.3 percent of GDP. This high level of external financing over the medium term will help additional reforms that are ongoing to sustain broad-based growth and to achieve the Millennium Development Goals by 2015. After 2015, external financing is expected to decline as a share of GDP to an average of about 10 percent of GDP for grants and about 2.5 percent of GDP for public loans.

\footnotetext{
${ }^{7}$ In 2008, the tax-to-GDP ratio is affected by the suspension of the fuel-related taxes to limit pressures on domestic prices arising from higher world prices. This suspension will end in 2009.
}

${ }^{8}$ IMF, 2007, "Mozambique: Evaluation of the Post-Reforms Tax System". 
Table 2. Mozambique: External Debt Sustainability Framework, Baseline Scenario, 2005-28 ${ }^{1}$

(Percent of GDP, unless otherwise indicated)

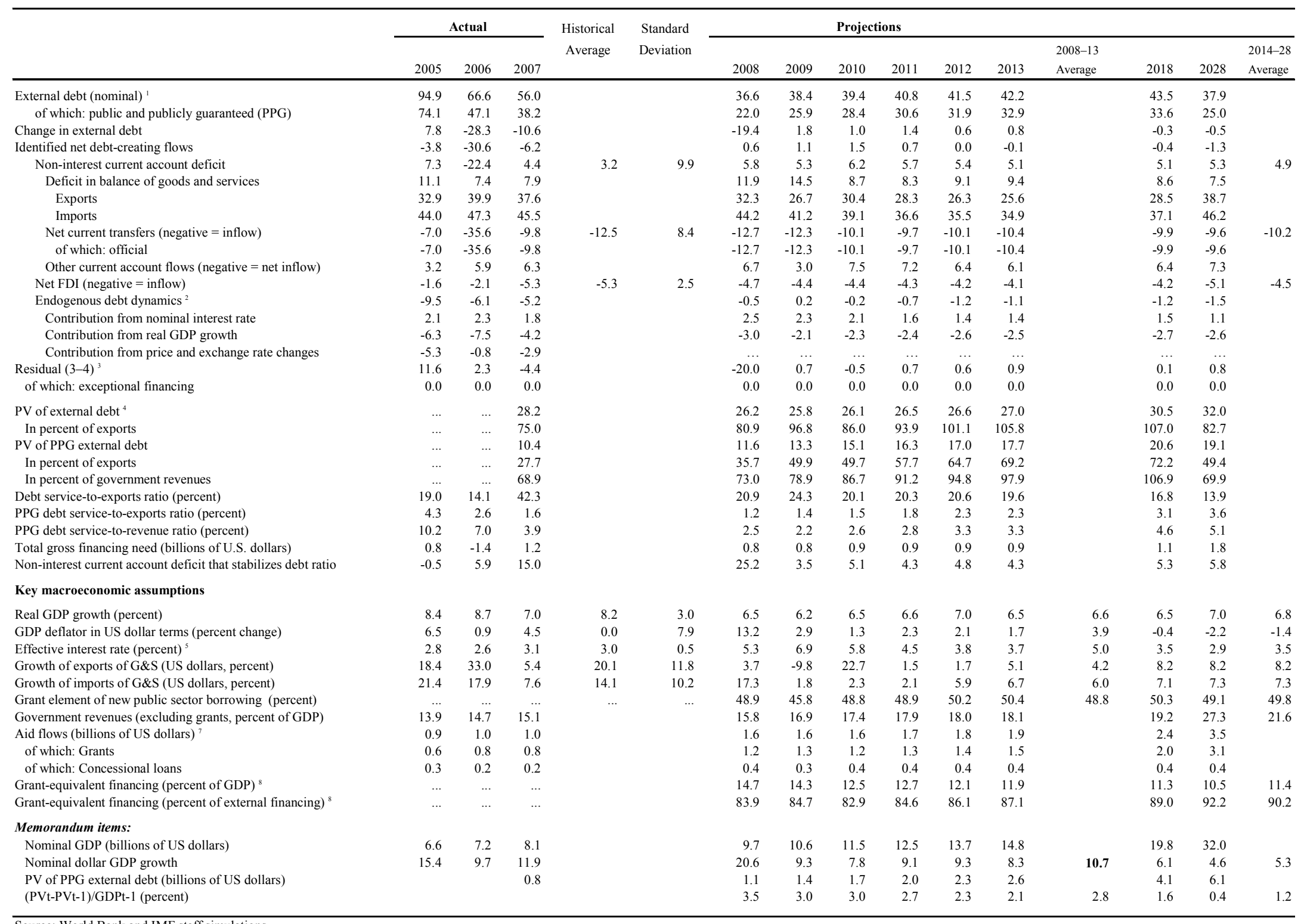

Source: World Bank and IMF staff simulations.

'Includes both public and private sector external debt.

Derived as $[\mathrm{r}-\mathrm{g}-\mathrm{r}(1+\mathrm{g})](1+\mathrm{g}+\mathrm{r}+\mathrm{gr})$ times previous period debt ratio, with $\mathrm{r}=$ nominal interest rate; $\mathrm{g}=$ real GDP growth rate, and $\mathrm{r}=$ growth rate of GDP deflator in U.S. dollar terms.

Includes exceptional financing (i.e., changes in arrears and debt relief); changes in gross foreign assets; and valuation adjustments. For projections also includes contribution from price and exchange rate changes.

Assumes that PV of private sector debt is equivalent to its face value.

Current-year interest payments divided by previous period debt stock.

Historical averages and standard deviations are generally derived over the past 10 years, subject to data availability.

Defined as grants, concessional loans, and debt relief.

${ }^{8}$ Grant-equivalent financing includes grants provided directly to the government and through new borrowing (difference between the face value and the PV of new debt). 
Table 3. Mozambique: Sensitivity Analysis for Key Indicators of Public and Publicly Guaranteed External Debt, 2008-28

\begin{tabular}{|c|c|c|c|c|c|c|c|c|}
\hline & \multicolumn{8}{|c|}{ Projections } \\
\hline & 2008 & 2009 & 2010 & 2011 & 2012 & 2013 & 2018 & 2028 \\
\hline \multicolumn{9}{|c|}{ PV of debt-to GDP ratio } \\
\hline Baseline & 12 & 13 & 15 & 16 & 17 & 18 & 21 & 19 \\
\hline \multicolumn{9}{|l|}{ A. Alternative Scenarios } \\
\hline A1. Key variables at their historical averages in $2008-28^{1}$ & 12 & 14 & 15 & 16 & 17 & 18 & 19 & 18 \\
\hline A2. New public sector loans on less favorable terms in $2008-28^{2}$ & 12 & 14 & 16 & 18 & 19 & 20 & 23 & 24 \\
\hline \multicolumn{9}{|l|}{ B. Bound Tests } \\
\hline B1. Real GDP growth at historical average minus one standard deviation in 2009-10 & 12 & 13 & 15 & 17 & 17 & 18 & 21 & 20 \\
\hline B2. Export value growth at historical average minus one standard deviation in $2009-10^{3}$ & 12 & 10 & 11 & 13 & 14 & 14 & 18 & 18 \\
\hline B3. U.S. dollar GDP deflator at historical average minus one standard deviation in 2009-10 & 12 & 15 & 19 & 20 & 21 & 22 & 25 & 24 \\
\hline B4. Net non-debt creating flows at historical average minus one standard deviation in $2009-10^{4}$ & 12 & 19 & 24 & 25 & 25 & 26 & 28 & 23 \\
\hline B5. Combination of B1-B4 using one-half standard deviation shocks & 12 & 13 & 15 & 16 & 17 & 18 & 21 & 21 \\
\hline B6. One-time 30 percent nominal depreciation relative to the baseline in $2009^{5}$ & 12 & 19 & 21 & 23 & 24 & 25 & 29 & 28 \\
\hline \multicolumn{9}{|c|}{ PV of debt-to-exports ratio } \\
\hline Baseline & 36 & 50 & 50 & 58 & 65 & 69 & 72 & 49 \\
\hline \multicolumn{9}{|l|}{ A. Alternative Scenarios } \\
\hline A1. Key variables at their historical averages in $2008-28^{1}$ & 36 & 51 & 49 & 57 & 64 & 69 & 67 & 47 \\
\hline A2. New public sector loans on less favorable terms in $2008-28^{2}$ & 36 & 51 & 53 & 62 & 71 & 77 & 82 & 62 \\
\hline \multicolumn{9}{|l|}{ B. Bound Tests } \\
\hline B1. Real GDP growth at historical average minus one standard deviation in 2009-10 & 36 & 50 & 50 & 57 & 64 & 69 & 72 & 51 \\
\hline B2. Export value growth at historical average minus one standard deviation in $2009-10^{3}$ & 36 & 32 & 35 & 42 & 49 & 53 & 58 & 45 \\
\hline B3. U.S. dollar GDP deflator at historical average minus one standard deviation in 2009-10 & 36 & 50 & 50 & 57 & 64 & 69 & 72 & 51 \\
\hline B4. Net non-debt creating flows at historical average minus one standard deviation in 2009-10 ${ }^{4}$ & 36 & 70 & 80 & 89 & 96 & 100 & 97 & 61 \\
\hline B5. Combination of B1-B4 using one-half standard deviation shocks & 36 & 36 & 37 & 43 & 49 & 53 & 57 & 42 \\
\hline B6. One-time 30 percent nominal depreciation relative to the baseline in $2009^{5}$ & 36 & 50 & 50 & 57 & 64 & 69 & 72 & 51 \\
\hline \multicolumn{9}{|c|}{ PV of debt-to-revenue ratio } \\
\hline Baseline & 73 & 79 & 87 & 91 & 95 & 98 & 107 & 70 \\
\hline \multicolumn{9}{|l|}{ A. Alternative Scenarios } \\
\hline A1. Key variables at their historical averages in $2008-28^{1}$ & 73 & 80 & 85 & 90 & 94 & 97 & 100 & 66 \\
\hline A2. New public sector loans on less favorable terms in $2008-28^{2}$ & 73 & 81 & 92 & 99 & 104 & 108 & 122 & 89 \\
\hline \multicolumn{9}{|l|}{ B. Bound Tests } \\
\hline B1. Real GDP growth at historical average minus one standard deviation in 2009-10 & 73 & 80 & 88 & 93 & 96 & 99 & 109 & 74 \\
\hline B2. Export value growth at historical average minus one standard deviation in $2009-10^{3}$ & 73 & 62 & 65 & 71 & 75 & 79 & 92 & 67 \\
\hline B3. U.S. dollar GDP deflator at historical average minus one standard deviation in 2009-10 & 73 & 88 & 106 & 112 & 116 & 120 & 131 & 89 \\
\hline B4. Net non-debt creating flows at historical average minus one standard deviation in $2009-10^{4}$ & 73 & 110 & 140 & 140 & 141 & 142 & 143 & 86 \\
\hline B5. Combination of B1-B4 using one-half standard deviation shocks & 73 & 78 & 84 & 90 & 95 & 99 & 111 & 79 \\
\hline B6. One-time 30 percent nominal depreciation relative to the baseline in $2009^{5}$ & 73 & 111 & 122 & 128 & 133 & 137 & 150 & 103 \\
\hline \multicolumn{9}{|c|}{ Debt service-to-exports ratio } \\
\hline Baseline & 1.2 & 1.4 & 1.5 & 1.8 & 2.3 & 2.3 & 3.1 & 3.6 \\
\hline \multicolumn{9}{|l|}{ A. Alternative Scenarios } \\
\hline A1. Key variables at their historical averages in $2008-28^{1}$ & 1.2 & 1.4 & 1.5 & 1.8 & 2.3 & 2.3 & 2.9 & 2.8 \\
\hline A2. New public sector loans on less favorable terms in $2008-28^{2}$ & 1.2 & 1.4 & 1.5 & 2.2 & 3.0 & 3.4 & 3.9 & 3.5 \\
\hline \multicolumn{9}{|l|}{ B. Bound Tests } \\
\hline B1. Real GDP growth at historical average minus one standard deviation in 2009-10 & 1.2 & 1.4 & 1.5 & 1.8 & 2.3 & 2.3 & 3.1 & 3.6 \\
\hline B2. Export value growth at historical average minus one standard deviation in $2009-10^{3}$ & 1.2 & 1.2 & 1.2 & 1.4 & 1.9 & 1.9 & 2.3 & 3.1 \\
\hline B3. U.S. dollar GDP deflator at historical average minus one standard deviation in 2009-10 & 1.2 & 1.4 & 1.5 & 1.8 & 2.3 & 2.3 & 3.1 & 3.6 \\
\hline B4. Net non-debt creating flows at historical average minus one standard deviation in 2009-10 ${ }^{4}$ & 1.2 & 1.4 & 1.9 & 2.5 & 3.0 & 3.0 & 4.3 & 4.5 \\
\hline B5. Combination of B1-B4 using one-half standard deviation shocks & 1.2 & 1.1 & 1.2 & 1.4 & 1.8 & 1.9 & 2.4 & 2.9 \\
\hline B6. One-time 30 percent nominal depreciation relative to the baseline in $2009^{5}$ & 1.2 & 1.4 & 1.5 & 1.8 & 2.3 & 2.3 & 3.1 & 3.6 \\
\hline Debt service-to & & & & & & & & \\
\hline Baseline & 2.5 & 2.2 & 2.6 & 2.8 & 3.3 & 3.3 & 4.6 & 5.1 \\
\hline A. Alternative Scenarios & & & & & & & & \\
\hline A1. Key variables at their historical averages in $2008-28^{1}$ & 2.5 & 2.3 & 2.6 & 2.8 & 3.4 & 3.3 & 4.3 & 3.9 \\
\hline A2. New public sector loans on less favorable terms in $2008-28^{2}$ & 2.5 & 2.2 & 2.7 & 3.5 & 4.4 & 4.7 & 5.8 & 4.9 \\
\hline B. Bound Tests & & & & & & & & \\
\hline B1. Real GDP growth at historical average minus one standard deviation in 2009-10 & 2.5 & 2.3 & 2.6 & 2.9 & 3.4 & 3.4 & 4.7 & 5.3 \\
\hline B2. Export value growth at historical average minus one standard deviation in $2009-10^{3}$ & 2.5 & 2.2 & 2.2 & 2.4 & 2.9 & 2.9 & 3.7 & 4.7 \\
\hline B3. U.S. dollar GDP deflator at historical average minus one standard deviation in 2009-10 & 2.5 & 2.5 & 3.2 & 3.5 & 4.1 & 4.1 & 5.6 & 6.3 \\
\hline B4. Net non-debt creating flows at historical average minus one standard deviation in $2009-10^{4}$ & 2.5 & 2.2 & 3.3 & 4.0 & 4.4 & 4.3 & 6.3 & 6.4 \\
\hline B5. Combination of B1-B4 using one-half standard deviation shocks & 2.5 & 2.4 & 2.7 & 2.9 & 3.5 & 3.5 & 4.7 & 5.5 \\
\hline B6. One-time 30 percent nominal depreciation relative to the baseline in $2009^{5}$ & 2.5 & 3.2 & 3.6 & 4.0 & 4.7 & 4.7 & 6.4 & 7.3 \\
\hline Memorandum item: & & & & & & & & \\
\hline Grant element assumed on residual financing (i.e., financing required above baseline) ${ }^{6}$ & 46.0 & 46.0 & 46.0 & 46.0 & 46.0 & 46.0 & 46.0 & 46.0 \\
\hline
\end{tabular}

Source: World Bank and IMF staff projections and simulations.

' Variables include real GDP growth, growth of GDP deflator (in U.S. dollar terms), non-interest current account in percent of GDP (excluding 2006, an outlier because of the MDRI grants), and non-debt creating flows.

${ }^{2}$ Assumes that the interest rate on new borrowing is by 2 percentage points higher than in the baseline., while grace and maturity periods are the same as in the baseline.

${ }^{3}$ Exports values are assumed to remain permanently at the lower level, but the current account as a share of GDP is assumed to return to its baseline level after the shock (implicitly assuming an offsetting adjustment in import levels).

${ }^{4}$ Includes official and private transfers and FDI.

${ }^{5}$ Depreciation is defined as percentage decline in dollar/local currency rate, such that it never exceeds 100 percent.

${ }^{6}$ Applies to all stress scenarios except for A2 (less favorable financing) in which the terms on all new financing are as specified in footnote 2 . 
Figure 1. Mozambique: Indicators of Public and Publicly Guaranteed External Debt under Alternatives Scenarios, 2008-28 ${ }^{1}$
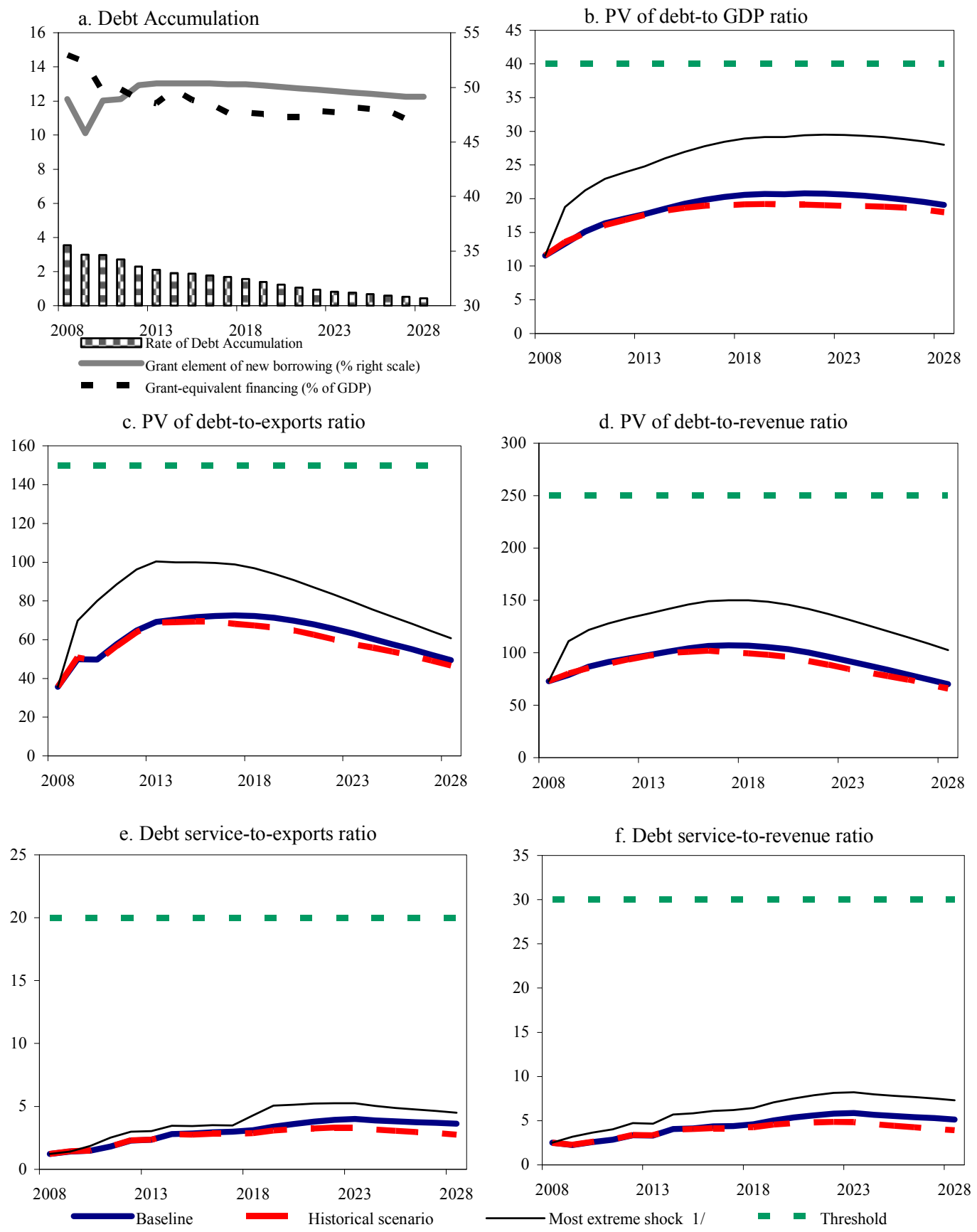

Source: World Bank and IMF staff projections and simulations.

${ }^{1}$ The most extreme stress test is the test that yields the highest ratio in 2018. In figure b. it corresponds to a one-time depreciation shock; in c. to a non-debt flows shock; in d. to a one-time depreciation shock; in e. to a Non-debt flows shock and in picture f. to a one-time depreciation shock 
Table 4. Mozambique: Public Sector Debt Sustainability Framework, Baseline Scenario, 2005-28

(Percent of GDP, unless otherwise indicated)

\begin{tabular}{|c|c|c|c|c|c|c|c|c|c|c|c|c|c|c|c|}
\hline & \multicolumn{3}{|c|}{ Actual } & \multirow[b]{2}{*}{ Average $^{5}$} & \multirow[b]{2}{*}{$\begin{array}{c}\text { Standard } \\
\text { Deviation }^{5}\end{array}$} & \multicolumn{5}{|l|}{ Estimate } & \multicolumn{5}{|c|}{ Projections } \\
\hline & 2005 & 2006 & 2007 & & & 2008 & 2009 & 2010 & 2011 & 2012 & 2013 & $\begin{array}{c}2008-13 \\
\text { Average }\end{array}$ & 2018 & 2028 & $\begin{array}{c}2014-28 \\
\text { Average }\end{array}$ \\
\hline Public sector debt ${ }^{1}$ & 81.0 & 53.5 & 42.1 & & & 24.3 & 28.6 & 30.7 & 32.6 & 33.6 & 34.4 & & 34.5 & 25.3 & \\
\hline of which: foreign-currency denominated & 74.1 & 47.1 & 38.2 & & & 22.0 & 25.9 & 28.4 & 30.6 & 31.9 & 32.9 & & 33.6 & 25.0 & \\
\hline Change in public sector debt & 11.1 & -27.5 & -11.4 & & & -17.8 & 4.3 & 2.1 & 1.9 & 1.0 & 0.7 & & -0.5 & -1.1 & \\
\hline Identified debt-creating flows & 2.9 & -7.6 & -6.9 & & & 0.4 & 3.6 & 1.7 & 1.6 & 1.0 & 0.7 & & -0.3 & -0.1 & \\
\hline Primary deficit & -0.9 & 0.8 & 3.2 & 1.0 & 3.1 & 4.0 & 4.9 & 4.0 & 3.5 & 3.2 & 2.8 & 3.7 & 1.9 & 1.2 & 1.5 \\
\hline Revenue and grants & 22.9 & 25.3 & 24.4 & & & 27.8 & 29.0 & 27.5 & 28.4 & 28.1 & 28.1 & & 29.2 & 36.9 & \\
\hline of which: grants & 9.0 & 10.6 & 9.3 & & & 12.0 & 12.1 & 10.1 & 10.5 & 10.1 & 10.1 & & 9.9 & 9.6 & \\
\hline Primary (noninterest) expenditure & 22.0 & 26.1 & 27.6 & & & 31.8 & 33.8 & 31.5 & 31.8 & 31.3 & 30.9 & & 31.0 & 38.1 & \\
\hline Automatic debt dynamics & 5.5 & -7.4 & -9.7 & & & -3.4 & -0.7 & -2.2 & -1.9 & -2.2 & -2.1 & & -2.2 & -1.3 & \\
\hline Contribution from interest rate/growth differential & -8.6 & -6.8 & -5.2 & & & -6.4 & -1.6 & -1.6 & -2.0 & -2.3 & -2.1 & & -1.5 & -0.9 & \\
\hline of which: contribution from average real interest rate & -3.2 & -0.3 & -1.7 & & & -3.9 & -0.2 & 0.1 & -0.1 & -0.1 & 0.0 & & 0.7 & 0.9 & \\
\hline of which: contribution from real GDP growth & -5.4 & -6.5 & -3.5 & & & -2.6 & -1.4 & -1.7 & -1.9 & -2.1 & -2.1 & & -2.1 & -1.7 & \\
\hline Contribution from real exchange rate depreciation & 14.1 & -0.6 & -4.5 & & & 3.0 & 0.9 & -0.6 & 0.1 & 0.1 & 0.0 & & $\ldots$ & $\ldots$ & \\
\hline Other identified debt-creating flows & -1.7 & -1.1 & -0.3 & & & -0.2 & -0.5 & 0.0 & 0.0 & 0.0 & 0.0 & & 0.0 & 0.0 & \\
\hline Privatization receipts (negative) & -0.3 & 0.0 & 0.0 & & & -0.1 & -0.4 & 0.0 & 0.0 & 0.0 & 0.0 & & 0.0 & 0.0 & \\
\hline Recognition of implicit or contingent liabilities & 0.0 & 0.0 & 0.0 & & & 0.0 & 0.0 & 0.0 & 0.0 & 0.0 & 0.0 & & 0.0 & 0.0 & \\
\hline Debt relief (HIPC and other) & -1.5 & -1.1 & -0.3 & & & -0.1 & -0.1 & 0.0 & 0.0 & 0.0 & 0.0 & & 0.0 & 0.0 & \\
\hline Other (specify, e.g. bank recapitalization) & 0.0 & 0.0 & 0.0 & & & 0.0 & 0.0 & 0.0 & 0.0 & 0.0 & 0.0 & & 0.0 & 0.0 & \\
\hline Residual, including asset changes & 8.2 & -19.9 & -4.5 & & & -18.2 & 0.6 & 0.4 & 0.4 & 0.0 & 0.0 & & -0.2 & -1.0 & \\
\hline \multicolumn{16}{|l|}{ Other Sustainability Indicators } \\
\hline PV of public sector debt & 6.9 & 6.3 & 13.5 & & & 14.0 & 16.8 & 17.9 & 18.9 & 19.5 & 19.9 & & 20.2 & 15.0 & \\
\hline of which: foreign-currency denominated & 0.0 & 0.0 & 9.7 & & & 11.7 & 14.1 & 15.6 & 16.9 & 17.7 & 18.4 & & 19.4 & 14.7 & \\
\hline of which: external & $\ldots$ & $\ldots$ & 9.7 & & & 11.7 & 14.1 & 15.6 & 16.9 & 17.7 & 18.4 & & 19.4 & 14.7 & \\
\hline $\mathrm{PV}$ of contingent liabilities (not included in public sector debt) & $\ldots$ & $\ldots$ & $\ldots$ & & & $\ldots$ & $\ldots$ & $\ldots$ & $\ldots$ & $\ldots$ & $\ldots$ & & $\ldots$ & $\ldots$ & \\
\hline Gross financing need $^{2}$ & 10.8 & 4.0 & 5.0 & & & 7.1 & 4.9 & 5.3 & 4.7 & 4.2 & 4.0 & & 3.2 & 2.9 & \\
\hline PV of public sector debt-to-revenue and grants ratio (percent) & 29.9 & 25.0 & 55.5 & & & 50.4 & 57.9 & 65.0 & 66.6 & 69.2 & 70.7 & & 69.3 & 40.8 & \\
\hline PV of public sector debt-to-revenue ratio (percent) & 49.1 & 43.0 & 89.6 & & & 88.5 & 99.4 & 102.5 & 105.6 & 108.2 & 110.0 & & 105.1 & 55.2 & \\
\hline of which: external ${ }^{3}$ & & & 63.9 & & & 74.1 & 83.5 & 89.4 & 94.5 & 98.5 & 101.7 & & 100.6 & 54.0 & \\
\hline Debt service-to-revenue and grants ratio (percent) ${ }^{4}$ & 9.0 & 6.8 & 5.1 & & & 3.5 & 2.4 & 3.0 & 3.0 & 3.2 & 3.1 & & 3.6 & 4.1 & \\
\hline Debt service-to-revenue ratio (percent) ${ }^{4}$ & 6.5 & 5.6 & 4.1 & & & 3.7 & 3.2 & 3.2 & 3.2 & 3.1 & 3.1 & & 2.7 & 1.5 & \\
\hline Primary deficit that stabilizes the debt-to-GDP ratio & -12.0 & 28.3 & 14.6 & & & 21.8 & 0.6 & 1.9 & 1.5 & 2.2 & 2.0 & & 2.4 & 2.3 & \\
\hline \multicolumn{16}{|l|}{ Key macroeconomic and fiscal assumptions } \\
\hline Real GDP growth (percent) & 8.4 & 8.7 & 7.0 & 8.2 & 3.0 & 6.5 & 6.2 & 6.5 & 6.6 & 7.0 & 6.5 & 6.6 & 6.5 & 7.0 & 6.8 \\
\hline Average nominal interest rate on forex debt (percent) & 0.5 & 0.4 & 0.5 & 1.4 & 1.2 & 0.7 & 1.1 & 1.2 & 1.2 & 1.2 & 1.2 & 1.1 & 1.2 & 1.2 & 1.2 \\
\hline Average real interest rate on domestic debt (percent) & 4.7 & 8.8 & 3.0 & 24.5 & 24.3 & -2.7 & 12.7 & 11.1 & 10.1 & 10.7 & 11.1 & 8.8 & 12.9 & 15.1 & 14.1 \\
\hline Real exchange rate depreciation (percent, + indicates depreciation) & 28.0 & 7.4 & -8.3 & 9.4 & 20.1 & 3.0 & & & & & $\ldots$ & $\ldots$ & $\ldots$ & $\ldots$ & $\ldots$ \\
\hline Inflation rate (GDP deflator, percent) & 6.5 & 0.9 & 4.5 & 0.0 & 7.9 & 13.2 & 2.9 & 1.3 & 2.3 & 2.1 & 1.7 & 3.9 & -0.4 & -2.2 & -1.4 \\
\hline Growth of real primary spending (deflated by GDP deflator, percent) & -0.1 & 0.3 & 0.1 & 0.1 & 0.2 & 0.2 & 0.1 & 0.0 & 0.1 & 0.1 & 0.1 & 0.1 & 0.1 & 0.1 & 0.1 \\
\hline Grant element of new external borrowing (percent) & $\ldots$ & $\ldots$ & $\ldots$ & $\ldots$ & $\ldots$ & 48.9 & 45.8 & 48.8 & 48.9 & 50.2 & 50.4 & 48.8 & 50.3 & 49.1 & $\ldots$ \\
\hline
\end{tabular}

Sources: Mozambican authorities; and World Bank and IMF staff estimates and projections.

1 [Indicate coverage of public sector, e.g., general government or nonfinancial public sector. Also whether net or gross debt is used.]

${ }^{2}$ Gross financing need is defined as the primary deficit plus debt service plus the stock of short-term debt at the end of the last period.

${ }^{3}$ Revenues excluding grants.

${ }^{4}$ Debt service is defined as the sum of interest and amortization of medium and long-term debt.

${ }^{5}$ Historical averages and standard deviations are generally derived over the past 10 years, subject to data availability. 
Table 5. Mozambique: Sensitivity Analysis for Key Indicators of Public Debt 2008-28

\begin{tabular}{|c|c|c|c|c|c|c|c|c|}
\hline & \multicolumn{8}{|c|}{ Projections } \\
\hline & 2008 & 2009 & 2010 & 2011 & 2012 & 2013 & 2018 & 2028 \\
\hline \multicolumn{9}{|c|}{ PV of Debt-to-GDP Ratio } \\
\hline Baseline & 14 & 17 & 18 & 19 & 19 & 20 & 20 & 15 \\
\hline \multicolumn{9}{|l|}{ A. Alternative scenarios } \\
\hline A1. Real GDP growth and primary balance are at historical averages & 14 & 13 & 11 & 10 & 9 & 8 & 5 & 4 \\
\hline A2. Primary balance is unchanged from 2008 & 14 & 16 & 17 & 19 & 20 & 21 & 29 & 46 \\
\hline A3. Permanently lower GDP growth ${ }^{1}$ & 14 & 17 & 18 & 20 & 21 & 22 & 27 & 42 \\
\hline \multicolumn{9}{|l|}{ B. Bound tests } \\
\hline B1. Real GDP growth is at historical average minus one standard deviations in $2009-10$ & 14 & 17 & 19 & 20 & 21 & 22 & 24 & 22 \\
\hline B2. Primary balance is at historical average minus one standard deviations in $2009-10$ & 14 & 16 & 17 & 18 & 19 & 19 & 20 & 15 \\
\hline B3. Combination of B1-B2 using one half standard deviation shocks & 14 & 15 & 14 & 16 & 16 & 17 & 17 & 11 \\
\hline B4. One-time 30 percent real depreciation in 2009 & 14 & 21 & 21 & 21 & 21 & 21 & 21 & 18 \\
\hline B5. 10 percent of GDP increase in other debt-creating flows in 2009 & 14 & 26 & 26 & 27 & 27 & 28 & 27 & 20 \\
\hline \multicolumn{9}{|c|}{ PV of Debt-to-Revenue Ratio ${ }^{2}$} \\
\hline Baseline & 50 & 58 & 65 & 67 & 69 & 71 & 69 & 41 \\
\hline \multicolumn{9}{|l|}{ A. Alternative scenarios } \\
\hline A1. Real GDP growth and primary balance are at historical averages & 50 & 45 & 42 & 37 & 34 & 30 & 19 & 12 \\
\hline A2. Primary balance is unchanged from 2008 & 50 & 55 & 62 & 66 & 71 & 76 & 98 & 124 \\
\hline A3. Permanently lower GDP growth ${ }^{1}$ & 50 & 58 & 67 & 69 & 74 & 77 & 90 & 109 \\
\hline \multicolumn{9}{|l|}{ B. Bound tests } \\
\hline B1. Real GDP growth is at historical average minus one standard deviations in $2009-10$ & 50 & 59 & 68 & 70 & 74 & 77 & 81 & 59 \\
\hline B2. Primary balance is at historical average minus one standard deviations in 2009-10 & 50 & 55 & 63 & 65 & 67 & 69 & 68 & 40 \\
\hline B3. Combination of B1-B2 using one half standard deviation shocks & 50 & 51 & 53 & 55 & 57 & 59 & 58 & 31 \\
\hline B4. One-time 30 percent real depreciation in 2009 & 50 & 74 & 77 & 75 & 76 & 76 & 72 & 48 \\
\hline B5. 10 percent of GDP increase in other debt-creating flows in 2009 & 50 & 89 & 96 & 96 & 97 & 98 & 92 & 54 \\
\hline
\end{tabular}

Debt Service-to-Revenue Ratio ${ }^{2}$

Baseline

\section{A. Alternative scenarios}

A1. Real GDP growth and primary balance are at historical averages

A2. Primary balance is unchanged from 2008

A3. Permanently lower GDP growth

$\begin{array}{rrrrrrrr}3 & 2 & 2 & 2 & 2 & 1 & 1 & 0 \\ 3 & 2 & 3 & 3 & 3 & 3 & 5 & 10 \\ 3 & 2 & 3 & 3 & 3 & 3 & 4 & 9\end{array}$

\section{B. Bound tests}

B1. Real GDP growth is at historical average minus one standard deviations in 2009-10

B2. Primary balance is at historical average minus one standard deviations in 2009-10

B3. Combination of B1-B2 using one half standard deviation shocks

B4. One-time 30 percent real depreciation in 2009

B5. 10 percent of GDP increase in other debt-creating flows in 2009

Sources: Mozambican authorities; and World Bank and IMF staff estimates and projections.

${ }^{1}$ Assumes that real GDP growth is at baseline minus one standard deviation divided by the length of the projection period.

${ }^{2}$ Revenues are defined inclusive of grants. 
Figure 2. Mozambique: Indicators of Public Debt Under Alternative Scenarios, 2008-28 ${ }^{1}$

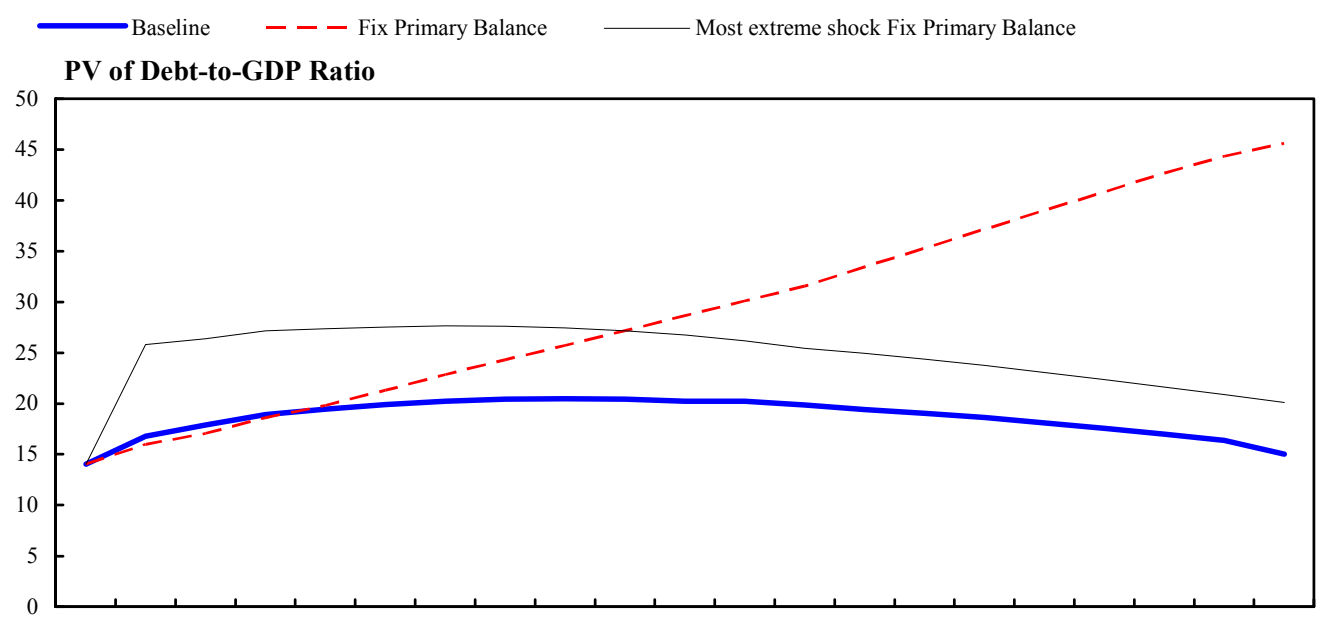

200820092010201120122013201420152016201720182019202020212022202320242025202620272028

PV of Debt-to-Revenue Ratio 2

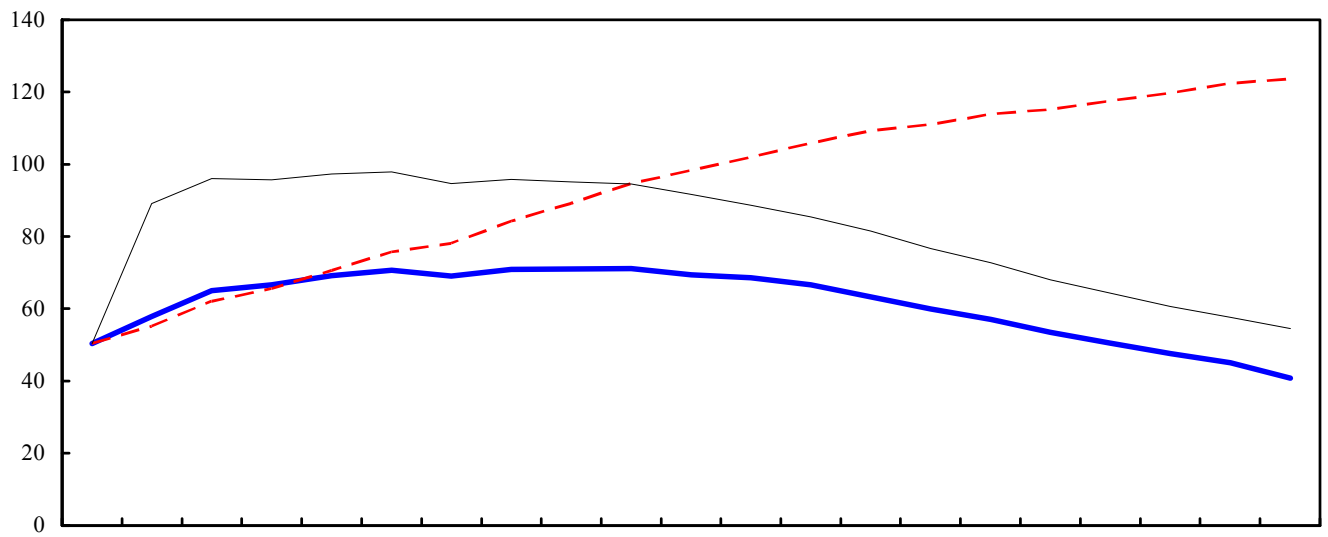

200820092010201120122013201420152016201720182019202020212022202320242025202620272028

Debt Service-to-Revenue Ratio 2

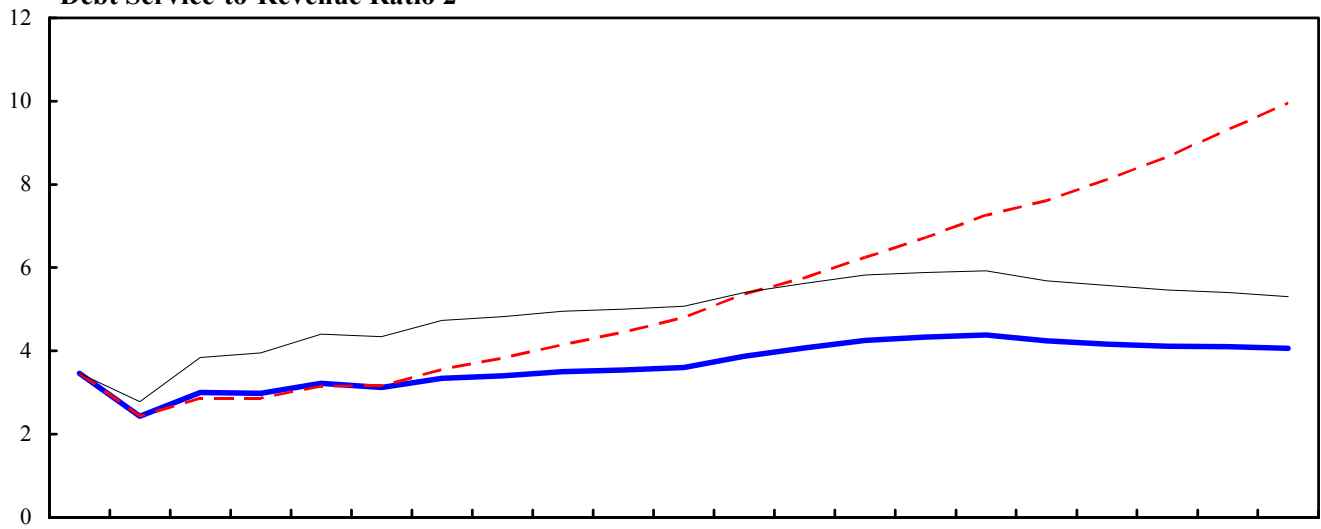

200820092010201120122013201420152016201720182019202020212022202320242025202620272028

Sources: Mozambican authorities; and World Bank and IMF staff estimates and projections.

${ }^{1}$ The most extreme stress test is the test that yields the highest ratio in 2018 .

${ }^{2}$ Revenues are defined inclusive of grants. 
Press Release No. 09/08

International Monetary Fund

FOR IMMEDIATE RELEASE

Washington, D.C. 20431 USA

January 16, 2009

\section{IMF Completes Third Review Under the Policy Support Instrument (PSI) for Mozambique}

The Executive Board of the International Monetary Fund (IMF) has completed the third review of Mozambique's economic performance under a three-year Policy Support Instrument (PSI). Mozambique's economic program with the IMF remains on track and all the quantitative and structural assesment criteria to end-June 2008 were met. The Board's decision was taken on a lapse of time basis. ${ }^{1}$

The PSI for Mozambique was approved on June 18, 2007 (See Press Release No 07/135) to support the nation's economic reform efforts. It is aimed at consolidating macroeconomic stability and at achieving sustained economic growth and poverty reduction through the pursuit of prudent macroeconomic policies as well as promoting structural reforms. The strategy to achieve this goal remains set in the Mozambican authorities' national poverty reduction strategy, Plano de Acção para Redução da Pobreza Absoluta (PARPA II).

\footnotetext{
${ }^{1}$ The Executive Board takes decisions under its lapse of time procedure when it is agreed by the Board that a proposal can be considered without convening formal discussions.
} 